\title{
FREE BIHOLOMORPHIC CLASSIFICATION OF NONCOMMUTATIVE DOMAINS
}

\author{
GELU POPESCU
}

\begin{abstract}
In this paper we develop a theory of free holomorphic functions on noncommutative Reinhardt domains $\mathbf{D}_{f}^{m}(\mathcal{H}) \subset B(\mathcal{H})^{n}$, generated by positive regular free holomorphic functions $f$ in $n$ noncommuting variables, and by positive integers $m \geq 1$, where $B(\mathcal{H})$ is the algebra of all bounded linear operators on a Hilbert space $\mathcal{H}$. Noncommutative Berezin transforms are used to study Hardy algebras $H^{\infty}\left(\mathbf{D}_{f, \text { rad }}^{m}\right)$ and domain algebras $A\left(\mathbf{D}_{f, \text { rad }}^{m}\right)$ associated with $\mathbf{D}_{f}^{m}(\mathcal{H})$, and compositions of free holomorphic functions.

We obtain noncommutative Cartan type results for formal power series, in several noncommuting indeterminates, which leave invariant the nilpotent parts of the corresponding domains. As a consequence, we characterize the set of all free biholomorphic functions $F: \mathbf{D}_{f}^{m}(\mathcal{H}) \rightarrow \mathbf{D}_{g}^{l}(\mathcal{H})$ with $F(0)=0$.

We show that the free biholomorphic classification of the domains $\mathbf{D}_{f}^{m}(\mathcal{H})$ is the same as the classification, up to unital completely isometric isomorphisms having completely contractive hereditary extension, of the corresponding noncommutative domain algebras $A\left(\mathbf{D}_{f, \text { rad }}^{m}\right)$. In particular, we prove that $\Psi: A\left(\mathbf{D}_{f, \mathrm{rad}}^{1}\right) \rightarrow A\left(\mathbf{D}_{g, \mathrm{rad}}^{1}\right)$ is a unital completely isometric isomorphism if and only if there is a free biholomorphic map $\varphi \in \operatorname{Bih}\left(\mathbf{D}_{g}^{1}, \mathbf{D}_{f}^{1}\right)$ such that

$$
\Psi(\chi)=\chi \circ \varphi, \quad \chi \in A\left(\mathbf{D}_{f, \mathrm{rad}}^{1}\right) .
$$

This implies that the noncommutative domains $\mathbf{D}_{f}^{1}(\mathcal{H})$ and $\mathbf{D}_{g}^{1}(\mathcal{H})$ are free biholomorphic equivalent if and only if the domain algebras $A\left(\mathbf{D}_{f, \mathrm{rad}}^{1}\right)$ and $A\left(\mathbf{D}_{g, \mathrm{rad}}^{1}\right)$ are completely isometrically isomorphic. Using the interaction between the theory of functions in several complex variables and our noncommutative theory, we provide several results concerning the free biholomorphic classification of the noncommutative domains $\mathbf{D}_{f}^{m}(\mathcal{H})$ and the classification, up to completely isometric isomorphisms, of the associated noncommutative domain (resp. Hardy) algebras. In particular, we characterize the unit ball of $B(\mathcal{H})^{n}$ among the noncommutative domains $\mathbf{D}_{f}^{m}(\mathcal{H})$, up to free biholomorphisms. We also obtain characterizations for the unitarily implemented isomorphisms of noncommutative Hardy (resp. domain) algebras in terms of free biholomorphic functions between the corresponding noncommutative domains.
\end{abstract}

\section{Contents}

\section{Introduction}

1. Free holomorphic functions on noncommutative Reinhardt domains

2. Noncommutative domain algebras and Hardy algebras

3. Compositions of free holomorphic functions

4. Free biholomorphic functions and noncommutative Cartan type results

5. Free biholomorphic classification of noncommutative domains

6. Isomorphisms of noncommutative Hardy algebras

References

Date: January 10, 2008.

2000 Mathematics Subject Classification. Primary: 46L52; 46T25. Secondary: 46L40; 47A56.

Key words and phrases. Noncommutative function theory; free holomorphic function; biholomorphic classification; automorphism group; Berezin transform; Hardy algebra; domain algebra; Fock space.

Research supported in part by an NSF grant. 


\section{INTRODUCTION}

In recent years, we have developed a noncommutative analytic function theory on the open unit ball

$$
\left[B(\mathcal{H})^{n}\right]_{1}:=\left\{\left(X_{1}, \ldots, X_{n}\right) \in B(\mathcal{H})^{n}:\left\|X_{1} X_{1}^{*}+\cdots+X_{n} X_{n}^{*}\right\|<1\right\}
$$

where $B(\mathcal{H})$ is the algebra of all bounded linear operators on a Hilbert space $\mathcal{H}$. We showed that several classical results from complex analysis and hyperbolic geometry have free analogues in this noncommutative multivariable setting (see [23, 25], 26, 27, 28, 30, 31]). Related to our work, we mention the papers [7, [9], 14], 15], [35], and [36, where several aspects of the theory of noncommutative analytic functions are considered in various settings. Some of the results concerning the noncommutative analytic function theory on the open unit ball $\left[B(\mathcal{H})^{n}\right]_{1}$ were extended (see Chapter 2 from 29]) to free holomorphic functions on the interiors of noncommutative ball-like domains

$$
\mathbf{D}_{p}^{1}(\mathcal{H}):=\left\{X:=\left(X_{1}, \ldots, X_{n}\right) \in B(\mathcal{H})^{n}: \Phi_{p, X}(I) \leq I\right\}
$$

where $\Phi_{p, X}(Y):=\sum_{k=1}^{\infty} \sum_{|\alpha|=k} a_{\alpha} X_{\alpha} Y X_{\alpha}^{*}$ for $Y \in B(\mathcal{H})$, and $p=\sum_{k=1}^{\infty} \sum_{|\alpha|=k} a_{\alpha} X_{\alpha}$ is a positive regular noncommutative polynomial, i.e., its coefficients are positive scalars and $a_{\alpha}>0$ if $\alpha \in \mathbb{F}_{n}^{+}$with $|\alpha|=1$. Here, $\mathbb{F}_{n}^{+}$is the unital free semigroup on $n$ generators $g_{1}, \ldots, g_{n}$ and the identity $g_{0}$. The length of $\alpha \in \mathbb{F}_{n}^{+}$is defined by $|\alpha|:=0$ if $\alpha=g_{0}$ and $|\alpha|:=k$ if $\alpha=g_{i_{1}} \cdots g_{i_{k}}$, where $i_{1}, \ldots, i_{k} \in\{1, \ldots, n\}$. If $X:=\left(X_{1}, \ldots, X_{n}\right) \in B(\mathcal{H})^{n}$ we denote $X_{\alpha}:=X_{i_{1}} \cdots X_{i_{k}}$ and $X_{g_{0}}:=I_{\mathcal{H}}$.

In [24, we studied more general noncommutative domains

$$
\mathbf{D}_{p}^{m}(\mathcal{H}):=\left\{X:=\left(X_{1}, \ldots, X_{n}\right) \in B(\mathcal{H})^{n}:\left(i d-\Phi_{p, X}\right)^{k}(I) \geq 0 \text { for } 1 \leq k \leq m\right\},
$$

where $m \geq 1$. We showed that each such a domain has a universal model $\left(W_{1}, \ldots, W_{n}\right)$ of weighted creation operators acting on the full Fock space $F^{2}\left(H_{n}\right)$ with $n$ generators. The study of $\mathbf{D}_{p}^{m}(\mathcal{H})$ is close related to the study of the weighted shifts $W_{1}, \ldots, W_{n}$, their joint invariant subspaces, and the representations of the algebras they generate: the domain algebra $\mathcal{A}_{n}\left(\mathbf{D}_{p}^{m}\right)$, the Hardy algebra $F_{n}^{\infty}\left(\mathbf{D}_{p}^{m}\right)$, and the $C^{*}$-algebra $C^{*}\left(W_{1}, \ldots, W_{n}\right)$.

The present paper is an attempt to develop a noncommutative analytic function theory on the radial parts

$$
\mathbf{D}_{p, \text { rad }}^{m}(\mathcal{H}):=\bigcup_{0 \leq r<1} r \mathbf{D}_{p}^{m}(\mathcal{H})
$$

of noncommutative starlike domains $\mathbf{D}_{p}^{m}(\mathcal{H})$, i.e., satisfying the condition $r \mathbf{D}_{p}^{m}(\mathcal{H}) \subset \mathbf{D}_{p}^{m}(\mathcal{H})$ for any $r \in[0,1)$. We mention that $\mathbf{D}_{p}^{1}(\mathcal{H})$ is always a starlike domain and $\mathbf{D}_{p, \text { rad }}^{1}(\mathcal{H})$ is equal to the interior of $\mathbf{D}_{p}^{1}(\mathcal{H})$. We also remark that if $q=X_{1}+\cdots+X_{n}$ and $m \geq 1$, then $\mathbf{D}_{q}^{m}(\mathcal{H})$ is a starlike domain which coincides with the set of all row contractions $\left(X_{1}, \ldots, X_{n}\right) \in\left[B(\mathcal{H})^{n}\right]_{1}^{-}$satisfying the positivity condition

$$
\sum_{k=0}^{m}(-1)^{k}\left(\begin{array}{c}
m \\
k
\end{array}\right) \sum_{|\alpha|=k} X_{\alpha} X_{\alpha}^{*} \geq 0 .
$$

The elements of the domain $\mathbf{D}_{q}^{m}(\mathcal{H})$ can be seen as multivariable noncommutative analogues of Agler's $m$-hypercontractions [1]. In this case, we also have that $\mathbf{D}_{q, \text { rad }}^{m}(\mathcal{H})$ is the interior of $\mathbf{D}_{q}^{m}(\mathcal{H})$.

We introduce the class of free holomorphic functions on the noncommutative radial domain $\mathbf{D}_{p, \mathrm{rad}}^{m}$, $m \geq 1$. A formal power series $F:=\sum_{\alpha \in \mathbb{F}_{n}^{+}} c_{\alpha} Z_{\alpha}, c_{\alpha} \in \mathbb{C}$, in $n$ noncommuting indeterminates $Z_{1}, \ldots, Z_{n}$ is called free holomorphic function (with scalar coefficients) on the noncommutative domain $\mathbf{D}_{p \text {,rad }}^{m}$ if its representation on any Hilbert space $\mathcal{H}$, i.e., the map $F: \mathbf{D}_{p \text {,rad }}^{m}(\mathcal{H}) \rightarrow B(\mathcal{H})$ given by

$$
F\left(X_{1}, \ldots, X_{n}\right):=\sum_{k=0}^{\infty} \sum_{|\alpha|=k} c_{\alpha} X_{\alpha}, \quad\left(X_{1}, \ldots, X_{n}\right) \in \mathbf{D}_{p, \text { rad }}^{m}(\mathcal{H}),
$$

is well-defined in the operator norm topology. The map $F$ is called free holomorphic function on $\mathbf{D}_{p, \text { rad }}^{m}(\mathcal{H})$. We remark that if $m=1$ and $p=X_{1}+\cdots+X_{n}$, then our definition coincides with the one for free holomorphic functions (see [23]) on the open unit ball of $B(\mathcal{H})^{n}$.

There is an important connection between the theory of free holomorphic functions on noncommutative domains $\mathbf{D}_{p \text {,rad }}^{m}(\mathcal{H})$ and the theory of holomorphic functions on the corresponding domains 
$\operatorname{Int}\left(\mathbf{D}_{p}^{m}\left(\mathbb{C}^{s}\right)\right) \subset \mathbb{C}^{n s^{2}}, s \in \mathbb{N}$, where Int denotes the interior. This is due to the fact that the representation of any free holomorphic function on a finite dimensional space $\left(\mathcal{H}=\mathbb{C}^{s}\right)$ is a holomorphic function in the classical sense. This connection will allow us to use the theory of functions in several complex variables ([12], [32], [34]) and our noncommutative theory in our attempt to classify the domains $\mathbf{D}_{p}^{m}(\mathcal{H})$ and the corresponding noncommutative domain (resp. Hardy) algebras (see Theorem 4.3 . Theorem 4.8, and Corollary 4.9). As in the case of biholomorphic maps between domains in $\mathbb{C}^{n}, n>1$, (see [11, 32]), we expect a great deal of rigidity for free biholomorphic functions between noncommutative domains.

In Section 1, we recall basic facts concerning the noncommutative Berezin transforms ([24]) associated with noncommutative domains $\mathbf{D}_{p}^{m}(\mathcal{H}), m \geq 1$, and characterize the algebra $\operatorname{Hol}_{\mathcal{E}}\left(\mathbf{D}_{p, \text { rad }}^{m}\right)$ of all free holomorphic functions on $\mathbf{D}_{p \text {,rad }}^{m}$, with operator-valued coefficients in $B(\mathcal{E})$.

In Section 2, we introduce the Hardy algebra $H^{\infty}\left(\mathbf{D}_{p, \text { rad }}^{m}\right)$ and the domain algebra $A\left(\mathbf{D}_{p \text {,rad }}^{m}\right)$ associated with the noncommutative domain $\mathbf{D}_{p \text {,rad }}^{m}, m \geq 1$. Let $H^{\infty}\left(\mathbf{D}_{p, \text { rad }}^{m}\right)$ denote the set of all elements $\varphi$ in $\operatorname{Hol}\left(\mathbf{D}_{p, \text { rad }}^{m}\right)$ such that $\|\varphi\|_{\infty}:=\sup \|\varphi(X)\|<\infty$, where the supremum is taken over all $n$-tuples $X \in \mathbf{D}_{p, \text { rad }}^{m}(\mathcal{H})$ and $\mathcal{H}$ is a separable infinite dimensional Hilbert space. We denote by $A\left(\mathbf{D}_{p, \text { rad }}^{m}\right)$ the set of all $\varphi$ in $\operatorname{Hol}\left(\mathbf{D}_{p, \text { rad }}^{m}\right)$ such that the mapping $\mathbf{D}_{p, \text { rad }}^{m}(\mathcal{H}) \ni X \mapsto \varphi(X) \in B(\mathcal{H})$ has a continuous extension to $\left[\mathbf{D}_{p, \text { rad }}^{m}(\mathcal{H})\right]^{-}=\mathbf{D}_{p}^{m}(\mathcal{H})$. It turns out that $H^{\infty}\left(\mathbf{D}_{p, \text { rad }}^{m}\right)$ and $A\left(\mathbf{D}_{p, \text { rad }}^{m}\right)$ are Banach algebras under pointwise multiplication and the norm $\|\cdot\|_{\infty}$. Using noncommutative Berezin transforms (24]), we identify the noncommutative algebra $F_{n}^{\infty}\left(\mathbf{D}_{p}^{m}\right)$ and the algebra $\mathcal{A}_{n}\left(\mathbf{D}_{p}^{m}\right)$ with the Hardy algebra $H^{\infty}\left(\mathbf{D}_{p \text {,rad }}^{m}\right)$ and the domain algebra $A\left(\mathbf{D}_{p, \text { rad }}^{m}\right)$, respectively (see Theorem 2.1] and Theorem 2.2). We recall (24]) that the Banach algebra $F_{n}^{\infty}\left(\mathbf{D}_{p}^{m}\right)$ is the sequential WOT-(resp. w*-)closure of all polynomials in $W_{1}, \ldots, W_{n}$, and the identity, while $\mathcal{A}_{n}\left(\mathbf{D}_{p}^{m}\right)$ is the norm closed algebra generated by the same operators. Hardy algebras of bounded free holomorphic functions with operator-valued coefficients are also discussed.

In Section 3, we present several results concerning the composition of free holomorphic functions on noncommutative domains $\mathbf{D}_{p, \text { rad }}^{m}$. These results are used, throughout this paper, to study free biholomorphic functions. Let $p$ and $g$ be positive regular noncommutative polynomials with $n$ and $q$ indeterminates, respectively, and let $m, l \geq 1$. A map $F: \mathbf{D}_{p}^{m}(\mathcal{H}) \rightarrow \mathbf{D}_{g}^{l}(\mathcal{H})$ is called free biholomorphic function if $F$ is a homeomorphism in the operator norm topology and $\left.F\right|_{\mathbf{D}_{p, \text { rad }}^{m}(\mathcal{H})}$ and $\left.F^{-1}\right|_{\mathbf{D}_{g, \text { rad }}^{l}(\mathcal{H})}$ are free homolorphic functions. In this case, the domains $\mathbf{D}_{p}^{m}(\mathcal{H})$ and $\mathbf{D}_{g}^{l}(\mathcal{H})$ are called free biholomorphic equivalent.

In Section 4, we obtain a noncommutative Cartan (5]) type result (see Theorem 4.5) characterizing the set $B i h_{0}\left(\mathbf{D}_{p}^{m}, \mathbf{D}_{g}^{l}\right)$ of all free biholomorphic functions $F: \mathbf{D}_{p}^{m}(\mathcal{H}) \rightarrow \mathbf{D}_{g}^{l}(\mathcal{H})$ with $F(0)=0$. Several consequences concerning the biholomorphic classification of noncommutative domains are obtained. In particular, we characterize the unit ball of $B(\mathcal{H})^{n}$ among the noncommutative domains $\mathbf{D}_{p}^{m}(\mathcal{H})$, up to free biholomorphisms.

In Section 5, we present the main result of this paper (see Theorem 5.1) which shows that the free biholomorphic classification of the noncommutative starlike domains $\mathbf{D}_{p}^{m}(\mathcal{H}) \subset B(\mathcal{H})^{n}$, generated by positive regular noncommutative polynomials $p$ and positive integers $m \geq 1$, is the same as the classification, up to unital completely isometric isomorphisms having completely contractive hereditary extension, of the corresponding noncommutative domain algebras $\mathcal{A}_{n}\left(\mathbf{D}_{p}^{m}\right) \simeq A\left(\mathbf{D}_{p, \text { rad }}^{m}\right)$. We show that $\Psi: A\left(\mathbf{D}_{p, \text { rad }}^{m}\right) \rightarrow A\left(\mathbf{D}_{g, \text { rad }}^{l}\right)$ is a unital completely isometric isomorphism having completely contractive hereditary extension if and only if there is a free biholomorphic map $\varphi \in \operatorname{Bih}\left(\mathbf{D}_{g}^{l}, \mathbf{D}_{p}^{m}\right)$ such that

$$
\Psi(\chi)=\chi \circ \varphi, \quad \chi \in A\left(\mathbf{D}_{p, \mathrm{rad}}^{m}\right) .
$$

In particular, we prove that the noncommutative domains $\mathbf{D}_{p}^{1}(\mathcal{H})$ and $\mathbf{D}_{g}^{1}(\mathcal{H})$ are free biholomorphic equivalent if and only if the domain algebras $\mathcal{A}_{n}\left(\mathbf{D}_{p}^{1}\right)$ and $\mathcal{A}_{q}\left(\mathbf{D}_{g}^{1}\right)$ are completely isometrically isomorphic. We mention that if $m=l=1$ and $p=g=X_{1}+\cdots+X_{n}$, we recover (with a different proof) the corresponding result from [30]. In the particular case when $n=m=l=1$ and $p=g=X$, we obtain the well-known result $([10])$ that the automorphisms of the disc algebra $A(\mathbb{D})$, of all bounded analytic functions on the open unit disc $\mathbb{D}:=\{z \in \mathbb{C}:|z|<1\}$ with continuous extensions to the closed disc, are the maps $\Theta_{\tau}(\varphi)=\varphi \circ \tau, \varphi \in A(\mathbb{D})$, where $\tau$ is a conformal automorphism of the unit disc $\mathbb{D}$.

Combining the main result of this paper with the Cartan type results from Section 4 , we obtain a characterization of all isomorphisms $\Psi: A\left(\mathbf{D}_{p, \text { rad }}^{m}\right) \rightarrow A\left(\mathbf{D}_{g, \text { rad }}^{l}\right)$ with $\varphi(0)=0$. We mention that, in the 
particular case when $m=l=1$, our result strengthens and provides a converse of the main result of Arias and Latrémolière (see Theorem 3.18 from [2]), who proved that if there is a completely isometric isomorphism between two noncommutative domain algebras $\mathcal{A}_{n}\left(\mathbf{D}_{p}^{1}\right)$ and $\mathcal{A}_{n}\left(\mathbf{D}_{g}^{1}\right)$ whose dual map fixes the origin, then the algebras are related by a linear relation of their generators.

In Section 6, we characterize the unitarily implemented isomorphisms between noncommutative Hardy algebras $H^{\infty}\left(\mathbf{D}_{p \text {,rad }}^{m}\right)$ and $H^{\infty}\left(\mathbf{D}_{g \text {,rad }}^{l}\right)$ in terms of free biholomorphic functions between the corresponding noncommutative domains. Similar results are deduced for noncommutative domain algebras. In particular, when $m=1$, we prove that the group $\left.\operatorname{Aut}_{u}\left(\mathcal{A}_{n}\left(\mathbf{D}_{p}^{1}\right)\right)\right)$ of all unitarily implemented automorphisms of the noncommutative domain algebra $\mathcal{A}_{n}\left(\mathbf{D}_{p}^{1}\right)$ is isomorphic to the group $A u t_{w}\left(\mathbf{D}_{p}^{1}\right)$ of all free biholomorphic functions $\varphi \in \operatorname{Aut}\left(\mathbf{D}_{p}^{1}\right)$ with the property that the model boundary function $\widetilde{\varphi}$ is a pure $n$-tuple, i.e., SOT- $\lim _{k \rightarrow \infty} \Phi_{p, \widetilde{\varphi}}^{k}(I)=0$, with $\operatorname{rank}\left[I-\Phi_{p, \widetilde{\varphi}}(I)\right]=1$ and the characteristic function $\Theta_{\widetilde{\varphi}}=0$.

We should mention that the results of this paper are presented in a more general setting, namely, when the polynomials $p$ and $g$ are replaced by positive regular free holomorphic functions on open balls $\left[B(\mathcal{H})^{n}\right]_{\gamma}, \gamma>0$.

\section{Free holomorphic functions on noncommutative Reinhardt domains}

We begin by setting up the notation and by recalling some basic facts concerning the noncommutative Berezin transforms associated with noncommutative domains $\mathbf{D}_{f}^{m}(\mathcal{H}), m \geq 1$. We characterize the algebra of all free holomorphic functions on $\mathbf{D}_{f, \text { rad }}^{m}$, with operator-valued coefficients, and discuss the connection between the theory of free holomorphic functions on noncommutative domains $\mathbf{D}_{f, \text { rad }}^{m}$ and the theory of holomorphic functions on domains in $\mathbb{C}^{d}$.

Let $f:=\sum_{\alpha \in \mathbb{F}_{n}^{+}} a_{\alpha} X_{\alpha}, \quad a_{\alpha} \in \mathbb{C}$, be a free holomorphic function on a ball $\left[B(\mathcal{H})^{n}\right]_{\gamma}$ for some $\gamma>0$. As shown in [23], this condition is equivalent to

$$
\limsup _{k \rightarrow \infty}\left(\sum_{|\alpha|=k}\left|a_{\alpha}\right|^{2}\right)^{1 / 2 k}<\infty .
$$

Throughout this paper, we assume that $a_{\alpha} \geq 0$ for any $\alpha \in \mathbb{F}_{n}^{+}, a_{g_{0}}=0$, and $a_{g_{i}}>0, i=1, \ldots, n$. A function $f$ satisfying all these conditions on the coefficients is called a positive regular free holomorphic function. We denote by $i d$ the identity map acting on the algebra of all bounded linear operators an a Hilbert space. Given $m, n \in \mathbb{N}:=\{1,2, \ldots\}$ and a positive regular free holomorphic function $f:=\sum_{\alpha \in \mathbb{F}_{n}^{+},|\alpha| \geq 1} a_{\alpha} X_{\alpha}$, we define the noncommutative domain

$$
\mathbf{D}_{f}^{m}(\mathcal{H}):=\left\{X:=\left(X_{1}, \ldots, X_{n}\right) \in B(\mathcal{H})^{n}:\left(i d-\Phi_{f, X}\right)^{k}(I) \geq 0 \text { for } 1 \leq k \leq m\right\},
$$

where $\Phi_{f, X}: B(\mathcal{H}) \rightarrow B(\mathcal{H})$ is defined by $\Phi_{f, X}(Y):=\sum_{k=1}^{\infty} \sum_{|\alpha|=k} a_{\alpha} X_{\alpha} Y X_{\alpha}^{*}, \quad Y \in B(\mathcal{H})$, and the convergence is in the week operator topology. $\mathbf{D}_{f}^{m}(\mathcal{H})$ can be seen as a noncommutative Reinhardt domain, i.e., $\left(e^{i \theta_{1}} X_{1}, \ldots, e^{i \theta_{n}} X_{n}\right) \in \mathbf{D}_{f}^{m}(\mathcal{H})$ for any $\left(X_{1}, \ldots, X_{n}\right) \in \mathbf{D}_{f}^{m}(\mathcal{H})$ and $\theta_{1}, \ldots, \theta_{n} \in \mathbb{R}$. We say that $\Phi_{f, X}$ is power bounded if there exists a constant $M>0$ such that $\left\|\Phi_{f, X}^{k}\right\| \leq M$ for any $k \in \mathbb{N}$. In particular, this is the case if $\Phi_{f, X}(I) \leq I$. We recall (see [24], Lemma 1.4) the following result. If $\Phi_{f, X}$ is a power bounded positive map and $m \in \mathbb{N}$, then

$$
\left(i d-\Phi_{f, X}\right)^{m}(I) \geq 0 \quad \text { if and only if } \quad\left(i d-\Phi_{f, X}\right)^{k}(I) \geq 0, \quad k=1,2, \ldots, m .
$$

Note that if $\left(X_{1}, \ldots, X_{n}\right) \in \mathbf{D}_{f}^{m}(\mathcal{H})$ then

$$
\left\|X_{1} X_{1}^{*}+\cdots+X_{n} X_{n}^{*}\right\| \leq \frac{1}{\min \left\{a_{\alpha}:|\alpha|=1\right\}} .
$$

Indeed, since $\Phi_{f, X}(I) \leq I$ and $a_{\alpha} \geq 0$, we deduce that

$$
\min \left\{a_{\alpha}:|\alpha|=1\right\}\left(X_{1} X_{1}^{*}+\cdots+X_{n} X_{n}^{*}\right) \leq \sum_{|\alpha| \geq 1} a_{\alpha} X_{\alpha} X_{\alpha}^{*} \leq I .
$$


Throughout this paper, unless otherwise specified, we assume that $\mathcal{H}$ is a separable infinite dimensional Hilbert space and $\mathbf{D}_{f}^{m}(\mathcal{H})$ is a closed (in the operator norm topology) starlike domain, i.e., $\left(r X_{1}, \ldots, r X_{n}\right) \in \mathbf{D}_{f}^{m}(\mathcal{H})$ for any $n$-tuple $\left(X_{1}, \ldots, X_{n}\right) \in \mathbf{D}_{f}^{m}(\mathcal{H})$ and $r \in[0,1)$. In particular, $\mathbf{D}_{f}^{m}(\mathcal{H})$ is path connected to 0 . Note that if $m=1$, then $\mathbf{D}_{f}^{1}(\mathcal{H})$ is always a closed starlike domain. This case was extensively studied in [29]. When $m \geq 2$, we point out the following class of noncommutative starlike domains.

Example 1.1. (24]) If $p\left(X_{1}, \ldots, X_{n}\right):=a_{1} X_{1}+\cdots+a_{n} X_{n}, a_{i}>0$, then $\mathbf{D}_{p}^{m}(\mathcal{H}), m \in \mathbb{N}$, is a closed starlike domain.

Now, we recall ( 24], 29]) a few facts concerning the noncommutative Berezin transforms associated with noncommutative domains $\mathbf{D}_{f}^{m}(\mathcal{H}), m \geq 1$. Let $H_{n}$ be an $n$-dimensional complex Hilbert space with orthonormal basis $e_{1}, e_{2}, \ldots, e_{n}$, where $n \in \mathbb{N}$, or $n=\infty$. Consider the full Fock space of $H_{n}$ defined by

$$
F^{2}\left(H_{n}\right):=\mathbb{C} 1 \oplus \bigoplus_{k \geq 1} H_{n}^{\otimes k},
$$

where $H_{n}^{\otimes k}$ is the (Hilbert) tensor product of $k$ copies of $H_{n}$. We denote $e_{\alpha}:=e_{i_{1}} \otimes \cdots \otimes e_{i_{k}}$ if $\alpha=g_{i_{1}} \cdots g_{i_{k}} \in \mathbb{F}_{n}^{+}$, where $i_{1}, \ldots, i_{k} \in\{1, \ldots, n\}$, and $e_{g_{0}}:=1$. Note that $\left\{e_{\alpha}\right\}_{\alpha \in \mathbb{F}_{n}^{+}}$is an orthonormal basis for $F^{2}\left(H_{n}\right)$. Define the left creation operators $S_{i}, i=1, \ldots, n$, acting on $F^{2}\left(H_{n}\right)$ by setting

$$
S_{i} \varphi:=e_{i} \otimes \varphi, \quad \varphi \in F^{2}\left(H_{n}\right) .
$$

Let $D_{i}: F^{2}\left(H_{n}\right) \rightarrow F^{2}\left(H_{n}\right), i=1, \ldots, n$, be the diagonal operators given by

$$
D_{i} e_{\alpha}:=\sqrt{\frac{b_{\alpha}^{(m)}}{b_{g_{i} \alpha}^{(m)}}} e_{\alpha}, \quad \alpha \in \mathbb{F}_{n}^{+},
$$

where

$$
b_{g_{0}}^{(m)}:=1 \quad \text { and } \quad b_{\alpha}^{(m)}:=\sum_{j=1}^{|\alpha|} \sum_{\substack{\gamma_{1}, \ldots, \gamma_{j}=\alpha \\
\left|\gamma_{1}\right| \geq 1, \ldots,\left|\gamma_{j}\right| \geq 1}} a_{\gamma_{1}} \cdots a_{\gamma_{j}}\left(\begin{array}{c}
j+m-1 \\
m-1
\end{array}\right) \quad \text { if } \alpha \in \mathbb{F}_{n}^{+},|\alpha| \geq 1 .
$$

Since $a_{g_{i}}>0$, Lemma 1.2 from [24] implies

$$
b_{g_{i} \alpha}^{(m)} \geq \sum_{\substack{\gamma \sigma=g_{i} \alpha \\ \sigma \in \mathbb{P}_{n}^{+},|\gamma| \geq 1}} b_{\sigma}^{(m)} a_{\gamma} \geq a_{g_{i}} b_{\alpha}^{(m)}, \quad i=1, \ldots, n
$$

and, consequently,

$$
\left\|D_{i}\right\|=\sup _{\alpha \in \mathbb{F}_{n}^{+}} \sqrt{\frac{b_{\alpha}^{(m)}}{b_{g_{i} \alpha}^{(m)}}} \leq \frac{1}{\sqrt{a_{g_{i}}}}, \quad i=1, \ldots, n .
$$

We define the weighted left creation operators $W_{i}: F^{2}\left(H_{n}\right) \rightarrow F^{2}\left(H_{n}\right), i=1, \ldots, n$, associated with the noncommutative domain $\mathbf{D}_{f}^{m}$ by setting $W_{i}:=S_{i} D_{i}$, where $S_{1}, \ldots, S_{n}$ are the left creation operators on the full Fock space $F^{2}\left(H_{n}\right)$. Note that

$$
W_{i} e_{\alpha}=\frac{\sqrt{b_{\alpha}^{(m)}}}{\sqrt{b_{g_{i} \alpha}^{(m)}}} e_{g_{i} \alpha}, \quad \alpha \in \mathbb{F}_{n}^{+},
$$

where the coefficients $b_{\alpha}^{(m)}, \alpha \in \mathbb{F}_{n}^{+}$, are given by relation (1.2). When necessary, we denote the weighted left creation operators $\left(W_{1}, \ldots, W_{n}\right)$ associated with $\mathbf{D}_{f}^{m}$ by $\left(W_{1}^{(f)}, \ldots, W_{n}^{(f)}\right)$. Using relation (1.3), one can easily see that

$$
W_{\beta} e_{\gamma}=\frac{\sqrt{b_{\gamma}^{(m)}}}{\sqrt{b_{\beta \gamma}^{(m)}}} e_{\beta \gamma} \quad \text { and } \quad W_{\beta}^{*} e_{\alpha}= \begin{cases}\frac{\sqrt{b_{\gamma}^{(m)}}}{\sqrt{b_{\alpha}^{(m)}}} e_{\gamma} & \text { if } \alpha=\beta \gamma \\ 0 & \text { otherwise }\end{cases}
$$


for any $\alpha, \beta \in \mathbb{F}_{n}^{+}$. According to Theorem 1.3 from [24, the weighted left creation operators $W_{1}, \ldots, W_{n}$ associated with $\mathbf{D}_{f}^{m}$ have the following properties:

(i) $\sum_{k=1}^{\infty} \sum_{|\beta|=k} a_{\beta} W_{\beta} W_{\beta}^{*} \leq I$, where the convergence is in the strong operator topology;

(ii) $\left(i d-\Phi_{f, W}\right)^{m}(I)=P_{\mathbb{C}}$, where $P_{\mathbb{C}}$ is the orthogonal projection from $F^{2}\left(H_{n}\right)$ onto $\mathbb{C} 1 \subset F^{2}\left(H_{n}\right)$, and $\lim _{p \rightarrow \infty} \Phi_{f, W}^{p}(I)=0$ in the strong operator topology.

Consequently, we deduce that $\left(W_{1}, \ldots, W_{n}\right) \in \mathbf{D}_{f}^{m}\left(F^{2}\left(H_{n}\right)\right)$. In [24], we proved that the $n$-tuple $\left(W_{1}, \ldots, W_{n}\right)$ plays the role of universal model for the noncommutative domain $\mathbf{D}_{f}^{m}$. We also introduced ([29, 24]) the domain algebra $\mathcal{A}_{n}\left(\mathbf{D}_{f}^{m}\right)$ associated with the noncommutative domain $\mathbf{D}_{f}^{m}$ to be the norm closure of all polynomials in $W_{1}, \ldots, W_{n}$, and the identity.

Following [19, 20], the noncommutative Hardy algebra $F_{n}^{\infty}\left(\mathbf{D}_{f}^{m}\right)$ was defined ([29, 24]) as follows. Let $\varphi\left(W_{1}, \ldots, W_{n}\right)=\sum_{\beta \in \mathbb{F}_{n}^{+}} c_{\beta} W_{\beta}$ be a formal Fourier representation such that $\sum_{\beta \in \mathbb{F}_{n}^{+}}\left|c_{\beta}\right|^{2} \frac{1}{b_{\beta}^{(m)}}<\infty$, where the coefficients $b_{\beta}^{(m)}, \beta \in \mathbb{F}_{n}^{+}$, are given by relation (1.2). Then $\sum_{\beta \in \mathbb{F}_{n}^{+}} c_{\beta} W_{\beta}(p) \in F^{2}\left(H_{n}\right)$ for any $p \in \mathcal{P}$, where $\mathcal{P}$ is the set of all polynomial in $F^{2}\left(H_{n}\right)$. If, in addition,

$$
\sup _{p \in \mathcal{P},\|p\| \leq 1}\left\|\sum_{\beta \in \mathbb{F}_{n}^{+}} c_{\beta} W_{\beta}(p)\right\|<\infty
$$

then there is a unique bounded operator acting on $F^{2}\left(H_{n}\right)$, which we denote also by $\varphi\left(W_{1}, \ldots, W_{n}\right)$, such that

$$
\varphi\left(W_{1}, \ldots, W_{n}\right) p=\sum_{\beta \in \mathbb{F}_{n}^{+}} c_{\beta} W_{\beta}(p), \quad p \in \mathcal{P} .
$$

The set of all operators $\varphi\left(W_{1}, \ldots, W_{n}\right) \in B\left(F^{2}\left(H_{n}\right)\right)$ satisfying the above-mentioned properties is denoted by $F_{n}^{\infty}\left(\mathbf{D}_{f}^{m}\right)$. We proved in [24] that the Banach algebra $F_{n}^{\infty}\left(\mathbf{D}_{f}^{m}\right) \subset B\left(F^{2}\left(H_{n}\right)\right)$ is the sequential SOT(resp. WOT-, $w^{*}-$ ) closure of all polynomials in $W_{1}, \ldots, W_{n}$, and the identity.

In the particular case when $m=1$ and $q=X_{1}+\cdots+X_{n}$, the algebras $\mathcal{A}_{n}\left(\mathbf{D}_{q}^{1}\right)$ and $F_{n}^{\infty}\left(\mathbf{D}_{q}^{1}\right)$ coincide with the noncommutative disc algebra $\mathcal{A}_{n}$ and the noncommutative analytic Toeplitz algebra $F_{n}^{\infty}$, respectively, which were introduced in [19] (see also [20, 21]) in connection with a noncommutative von Neumann type inequality and have been extensively studied in the last two decades. For the classical von Neumann inequality we refer the reader to [37, [33], [17, and [16].

The joint spectral radius of an $n$-tuple $T:=\left(T_{1}, \ldots, T_{n}\right) \in \mathbf{D}_{f}^{m}(\mathcal{H})$ is defined by

$$
r_{f}\left(T_{1}, \ldots, T_{n}\right):=\lim _{k \rightarrow \infty}\left\|\Phi_{f, T}^{k}(I)\right\|^{1 / 2 k},
$$

where $\Phi_{f, X}: B(\mathcal{H}) \rightarrow B(\mathcal{H})$ is given by $\Phi_{f, X}(Y):=\sum_{k=1}^{\infty} \sum_{|\alpha|=k} a_{\alpha} X_{\alpha} Y X_{\alpha}^{*}, \quad Y \in B(\mathcal{H})$. For each $T:=\left(T_{1}, \ldots, T_{n}\right) \in \mathbf{D}_{f}^{m}(\mathcal{H})$ with $r_{f}\left(T_{1}, \ldots, T_{n}\right)<1$, we introduced (see [24]) the noncommutative Berezin transform at $T$ as the map $\mathbf{B}_{T}: B\left(F^{2}\left(H_{n}\right)\right) \rightarrow B(\mathcal{H})$ defined by

$$
\left\langle\mathbf{B}_{T}[g] x, y\right\rangle:=\left\langle\left(I-\sum_{|\alpha| \geq 1} \bar{a}_{\tilde{\alpha}} \Lambda_{\alpha}^{*} \otimes T_{\tilde{\alpha}}\right)^{-m}\left(g \otimes \Delta_{f, m, T}^{2}\right)\left(I-\sum_{|\alpha| \geq 1} a_{\tilde{\alpha}} \Lambda_{\alpha} \otimes T_{\tilde{\alpha}}^{*}\right)^{-m}(1 \otimes x), 1 \otimes y\right\rangle
$$

where $g \in B\left(F^{2}\left(H_{n}\right)\right), \Delta_{f, m, T}:=\left[\left(i d-\Phi_{f, T}\right)^{m}(I)\right]^{1 / 2}$ and $x, y \in \mathcal{H}$. We should add that the $n$-tuple $\left(\Lambda_{1}, \ldots, \Lambda_{n}\right)$ is the universal model associated with the noncommutative domain $\mathbf{D}_{\tilde{f}}^{m}$, where $\widetilde{f}=\sum a_{\tilde{\alpha}} Z_{\alpha}$ and $\widetilde{\alpha}:=g_{i_{k}} \cdots g_{i_{1}}$ denotes the reverse of $\alpha=g_{i_{1}} \cdots g_{i_{k}} \in \mathbb{F}_{n}^{+}$. We remark that in the particular case when $n=1, m=1, f=Z, \mathcal{H}=\mathbb{C}$, and $T=\lambda \in \mathbb{D}$, we recover the Berezin transform 3 of a bounded linear operator on the Hardy space $H^{2}(\mathbb{D})$, i.e.,

$$
\mathbf{B}_{\lambda}[g]=\left(1-|\lambda|^{2}\right)\left\langle g k_{\lambda}, k_{\lambda}\right\rangle, \quad g \in B\left(H^{2}(\mathbb{D})\right),
$$

where $k_{\lambda}(z):=(1-\bar{\lambda} z)^{-1}$ and $z, \lambda \in \mathbb{D}$. The noncommutative Berezin transform will play an important role in this paper. 
The extended noncommutative Berezin transform $\widetilde{\mathbf{B}}_{T}$ at $T \in \mathbf{D}_{f}^{m}(\mathcal{H})$ is defined by

$$
\widetilde{\mathbf{B}}_{T}[g]:=K_{f, T}^{(m)^{*}}\left(g \otimes I_{\mathcal{H}}\right) K_{f, T}^{(m)}, \quad g \in B\left(F^{2}\left(H_{n}\right)\right)
$$

where the noncommutative Berezin kernel $K_{f, T}^{(m)}: \mathcal{H} \rightarrow F^{2}\left(H_{n}\right) \otimes \overline{\Delta_{f, m, T}(\mathcal{H})}$ is given by

$$
K_{f, T}^{(m)} h=\sum_{\alpha \in \mathbb{F}_{n}^{+}} \sqrt{b_{\alpha}^{(m)}} e_{\alpha} \otimes \Delta_{f, m, T} T_{\alpha}^{*} h, \quad h \in \mathcal{H} .
$$

We recall that the noncommutative Berezin transforms $\widetilde{\mathbf{B}}_{T}$ and $\mathbf{B}_{T}$ coincide for any $n$-tuple of operators $T:=\left(T_{1}, \ldots, T_{n}\right) \in \mathbf{D}_{f}^{m}(\mathcal{H})$ with joint spectral radius $r_{f}\left(T_{1}, \ldots, T_{n}\right)<1$. In the particular case when $m=1$ and $f=X_{1}+\cdots+X_{n}$, the extended Berezin transform coincides with the noncommutative Poisson transform (see [21], 22]).

Let $T:=\left(T_{1}, \ldots, T_{n}\right)$ be an $n$-tuple of operators in the noncommutative starlike domain $\mathbf{D}_{f}^{m}(\mathcal{H})$ and let $\mathcal{S}:=\overline{\operatorname{span}}\left\{W_{\alpha} W_{\beta}^{*} ; \alpha, \beta \in \mathbb{F}_{n}^{+}\right\}$. Then there is a unital completely contractive linear map $\mathcal{B}_{T}: \mathcal{S} \rightarrow B(\mathcal{H})$, called the noncommutative Berezin transform at $T$ on the operator system $\mathcal{S}$, such that

$$
\mathcal{B}_{T}(g)=\lim _{r \rightarrow 1} \mathbf{B}_{r T}[g], \quad g \in \mathcal{S}
$$

where the limit exists in the operator norm topology of $B(\mathcal{H})$, and

$$
\mathcal{B}_{T}\left(W_{\alpha} W_{\beta}^{*}\right)=T_{\alpha} T_{\beta}^{*}, \quad \alpha, \beta \in \mathbb{F}_{n}^{+} .
$$

In particular, we have the following von Neumann type inequality:

$$
\left\|\sum_{\alpha, \beta \in \Lambda} T_{\alpha} T_{\beta}^{*} \otimes C_{\alpha, \beta}\right\| \leq\left\|\sum_{\alpha, \beta \in \Lambda} W_{\alpha} W_{\beta}^{*} \otimes C_{\alpha, \beta}\right\|
$$

for any finite set $\Lambda \subset \mathbb{F}_{n}^{+}$and $C_{\alpha, \beta} \in B(\mathcal{E})$, where $\mathcal{E}$ is a Hilbert space. Moreover, the restriction of $\mathcal{B}_{T}$ to the domain algebra $\mathcal{A}_{n}\left(\mathbf{D}_{f}^{m}\right)$ is a completely contractive homomorphism.

After these preliminaries on noncommutative Berezin transforms, we are ready to introduce the class of free holomorphic functions on the noncommutative radial domain $\mathbf{D}_{f, \text { rad }}^{m}$, with operator-valued coefficients.

For $0<r<1$, we define the domain $\mathbf{D}_{f, r}^{m}(\mathcal{H}) \subset B(\mathcal{H})^{n}$ by setting

$$
\mathbf{D}_{f, r}^{m}(\mathcal{H}):=\left\{\left(Y_{1}, \ldots, Y_{n}\right) \in B(\mathcal{H})^{n}:\left(\frac{1}{r} Y_{1}, \ldots, \frac{1}{r} Y_{n}\right) \in \mathbf{D}_{f}^{m}(\mathcal{H})\right\}=r \mathbf{D}_{f}^{m}(\mathcal{H}) .
$$

Since $\mathbf{D}_{f}^{m}(\mathcal{H})$ is a starlike domain, we have $\mathbf{D}_{f, r}^{m}(\mathcal{H}) \subset \mathbf{D}_{f}^{m}(\mathcal{H})$ for any $r \in[0,1)$. Consider the radial domain

$$
\mathbf{D}_{f, \mathrm{rad}}^{m}(\mathcal{H}):=\bigcup_{0 \leq r<1} \mathbf{D}_{f, r}^{m}(\mathcal{H})=\bigcup_{0 \leq r<1} r \mathbf{D}_{f}^{m}(\mathcal{H}) .
$$

Since, throughout the paper, $\mathbf{D}_{f}^{m}(\mathcal{H})$ is assumed to be closed in the operator norm topology, we have $\mathbf{D}_{f, \text { rad }}^{m}(\mathcal{H})^{-}=\mathbf{D}_{f}^{m}(\mathcal{H})$. Note that the interior of $\mathbf{D}_{f}^{m}(\mathcal{H})$, which we denote by $\operatorname{Int}\left(\mathbf{D}_{f}^{m}(\mathcal{H})\right)$, is a subset of $\mathbf{D}_{f, \text { rad }}^{m}(\mathcal{H})$. In particular, if $q$ is any positive regular noncommutative polynomial, then

$$
\operatorname{Int}\left(\mathbf{D}_{q}^{1}(\mathcal{H})\right)=\mathbf{D}_{q, \mathrm{rad}}^{1}(\mathcal{H}) \quad \text { and } \quad \overline{\operatorname{Int}\left(\mathbf{D}_{q}^{1}(\mathcal{H})\right)}=\mathbf{D}_{q}^{1}(\mathcal{H})
$$

A similar result holds, for example, for the domains $\mathbf{D}_{f}^{m}(\mathcal{H})$ if $m \geq 1$ and $f=\sum_{i=1}^{n} a_{i} X_{i}$ with $a_{i}>0$.

Let $\mathcal{E}$ be a separable Hilbert space. A formal power series $G:=\sum_{\alpha \in \mathbb{F}_{n}^{+}} Z_{\alpha} \otimes C_{(\alpha)}$, where $C_{(\alpha)} \in B(\mathcal{E})$, is called free holomorphic function on the noncommutative domain $\mathbf{D}_{f \text {,rad }}^{m}$, with coefficients in $B(\mathcal{E})$, if its representation on any Hilbert space $\mathcal{H}$, i.e., the mapping $G: \mathbf{D}_{\text {f,rad }}^{m}(\mathcal{H}) \rightarrow B(\mathcal{H}) \bar{\otimes}_{\min } B(\mathcal{E})$ given by

$$
G\left(X_{1}, \ldots, X_{n}\right):=\sum_{k=0}^{\infty} \sum_{|\alpha|=k} X_{\alpha} \otimes C_{(\alpha)}, \quad\left(X_{1}, \ldots, X_{n}\right) \in \mathbf{D}_{f, \text { rad }}^{m}(\mathcal{H}),
$$


is well-defined in the operator norm topology. The map $G$ is called free holomorphic function on $\mathbf{D}_{f, \text { rad }}^{m}(\mathcal{H})$. We remark that if $m=1$ and $f=X_{1}+\cdots+X_{n}$, then our definition coincides with the one for free holomorphic functions (see [23]) on the open unit ball of $B(\mathcal{H})^{n}$.

Our first result is the following characterization of free holomorphic functions on the noncommutative domain $\mathbf{D}_{f, \text { rad }}^{m}$, with operator-valued coefficients.

Theorem 1.2. Let $G\left(Z_{1}, \ldots, Z_{n}\right):=\sum_{\alpha \in \mathbb{F}_{n}^{+}} Z_{\alpha} \otimes C_{(\alpha)}$ be a formal power series with operator-valued coefficients $C_{(\alpha)} \in B(\mathcal{E})$, and let $\mathcal{H}$ be a separable infinite dimensional Hilbert space. Then the following statements are equivalent:

(i) $G$ is a free holomorphic function on the noncommutative domain $\mathbf{D}_{f, \text { rad }}^{m}$.

(ii) For any $r \in[0,1)$, the series

$$
G\left(r W_{1}, \ldots, r W_{n}\right):=\sum_{k=0}^{\infty} \sum_{|\alpha|=k} r^{|\alpha|} W_{\alpha} \otimes C_{(\alpha)}
$$

is convergent in the operator norm topology, where $\left(W_{1}, \ldots, W_{n}\right)$ is the universal model associated with the noncommutative domain $\mathbf{D}_{f}^{m}$.

(iii) The inequality

$$
\limsup _{k \rightarrow \infty}\left\|\sum_{|\alpha|=k} \frac{1}{b_{\alpha}^{(m)}} C_{(\alpha)}^{*} C_{(\alpha)}\right\|^{\frac{1}{2 k}} \leq 1,
$$

holds, where the coefficients $b_{\alpha}^{(m)}$ are given by relation (1.2).

(iv) For any $r \in[0,1)$, the series $\sum_{k=0}^{\infty}\left\|\sum_{|\alpha|=k} r^{|\alpha|} W_{\alpha} \otimes C_{(\alpha)}\right\|$ is convergent.

(v) For any $\left(X_{1}, \ldots, X_{n}\right) \in \mathbf{D}_{f, \text { rad }}^{m}(\mathcal{H})$, the series $\sum_{k=0}^{\infty}\left\|\sum_{|\alpha|=k} X_{\alpha} \otimes C_{(\alpha)}\right\|$ is convergent.

Proof. Since $\left(W_{1}, \ldots, W_{n}\right) \in \mathbf{D}_{f}^{m}\left(F^{2}\left(H_{n}\right)\right)$, the implication $(i) \Longrightarrow$ (ii) is obvious. To prove that (ii) $\Longrightarrow($ iii $)$, we assume that

$$
\limsup _{k \rightarrow \infty}\left\|\sum_{|\alpha|=k} \frac{1}{b_{\alpha}^{(m)}} C_{(\alpha)}^{*} C_{(\alpha)}\right\|^{\frac{1}{2 k}}>\gamma>1 .
$$

Then there is $k$ as large as necessary such that $\left\|\sum_{|\alpha|=k} \frac{1}{b_{\alpha}^{(m)}} C_{(\alpha)}^{*} C_{(\alpha)}\right\|>\gamma^{2 k}$. Let $r$ be such that $\frac{1}{\gamma}<r<1$ and let $h \in \mathcal{E}$. Note that, using relation (1.4), we have

$$
\begin{aligned}
\left\|\sum_{|\beta|=k} r^{|\beta|} W_{\beta}(1) \otimes C_{(\beta)} h\right\| & =\left\|\sum_{|\beta|=k} r^{|\beta|} \frac{1}{\sqrt{b_{\beta}^{(m)}}} e_{\beta} \otimes C_{(\beta)} h\right\| \\
& =\left(r^{2 k}\left\langle\sum_{|\beta|=k} \frac{1}{b_{\beta}^{(m)}} C_{(\beta)}^{*} C_{(\beta)} h, h\right\rangle\right)^{1 / 2} .
\end{aligned}
$$

Hence, we deduce that

$$
\left\|\sum_{|\beta|=k} r^{|\beta|} W_{\beta} \otimes C_{(\beta)}\right\| \geq r^{k}\left\|\sum_{|\alpha|=k} \frac{1}{b_{\alpha}^{(m)}} C_{(\alpha)}^{*} C_{(\alpha)}\right\|^{1 / 2}>(r \gamma)^{k} .
$$

Since $r \gamma>1$, the series $\sum_{k=0}^{\infty} \sum_{|\beta|=k} r^{|\beta|} W_{\beta} \otimes C_{(\beta)}$ is divergent in the operator norm topology. 
Now we prove that $($ iii $) \Longrightarrow($ iv). Assume that item (iii) holds and let $0<r<\rho<1$. Then, we can find $m_{0} \in \mathbb{N}$ such that

$$
\left\|\sum_{|\alpha|=k} \frac{1}{b_{\alpha}^{(m)}} C_{(\alpha)}^{*} C_{(\alpha)}\right\|<\left(\frac{1}{\rho}\right)^{2 k} \quad \text { for any } k \geq m_{0} .
$$

According to Lemma 1.1 from [24] and relation (1.4), the operators $\left\{W_{\beta}\right\}_{|\beta|=k}$ have orthogonal ranges and

$$
\left\|W_{\beta} x\right\| \leq \frac{1}{\sqrt{b_{\beta}^{(m)}}} M_{|\beta|, m}\|x\|, \quad x \in F^{2}\left(H_{n}\right),
$$

where $M_{|\beta|, m}:=\left(\begin{array}{c}|\beta|+m-1 \\ m-1\end{array}\right)$. Consequently, we have

$$
\left\|\sum_{|\beta|=k} b_{\beta}^{(m)} W_{\beta} W_{\beta}^{*}\right\| \leq\left(\begin{array}{c}
k+m-1 \\
m-1
\end{array}\right) \quad \text { for any } \quad k=0,1, \ldots .
$$

Hence, and using relation (1.8), we deduce that

$$
\begin{aligned}
\sum_{k=0}^{\infty} r^{k}\left\|\sum_{|\beta|=k} W_{\beta} \otimes C_{(\beta)}\right\| & \leq \sum_{k=0}^{\infty} r^{k}\left\|\sum_{|\beta|=k} \frac{1}{b_{\beta}^{(m)}} C_{(\beta)}^{*} C_{(\beta)}\right\|^{1 / 2}\left\|\sum_{|\beta|=k} b_{\beta}^{(m)} W_{\beta} W_{\beta}^{*}\right\|^{1 / 2} \\
& \leq \sum_{k=0}^{\infty}\left\|\sum_{|\beta|=k} \frac{1}{b_{\beta}^{(m)}} C_{(\beta)}^{*} C_{(\beta)}\right\|^{1 / 2} r^{k}\left(\begin{array}{c}
k+m-1 \\
m-1
\end{array}\right)^{1 / 2} \\
& \leq \sum_{k=0}^{\infty}\left(\frac{r}{\rho}\right)^{k}\left(\begin{array}{c}
k+m-1 \\
m-1
\end{array}\right)^{1 / 2}<\infty
\end{aligned}
$$

which proves (iv). Since the implication $(v) \Longrightarrow(i)$ is obvious, it remains to prove that $(i v) \Longrightarrow(v)$. To this end, assume that item $(i v)$ holds and let $\left(X_{1} \ldots, X_{n}\right) \in \mathbf{D}_{f, \text { rad }}^{m}(\mathcal{H})$. Then there exists $r \in(0,1)$ such that $\left(\frac{1}{r} X_{1}, \ldots, \frac{1}{r} X_{n}\right) \in \mathbf{D}_{f}^{m}(\mathcal{H})$ and, using the noncommutative von Neumann inequality (1.7), we deduce that

$$
\left\|\sum_{|\alpha|=k} X_{\alpha} \otimes C_{(\alpha)}\right\| \leq\left\|\sum_{|\alpha|=k} r^{|\alpha|} W_{\alpha} \otimes C_{(\alpha)}\right\| .
$$

Hence, the series $\sum_{k=0}^{\infty}\left\|\sum_{|\alpha|=k} X_{\alpha} \otimes C_{(\alpha)}\right\|$ is convergent and, therefore, item $(v)$ holds. The proof is complete.

We remark that the coefficients of a free holomorphic function are uniquely determined by its representation on an infinite dimensional Hilbert space. Indeed, let $F: \mathbf{D}_{f, \text { rad }}^{m}(\mathcal{H}) \rightarrow B(\mathcal{H}) \bar{\otimes}_{\min } B(\mathcal{E})$ be a free holomorphic function on $\mathbf{D}_{f, \text { rad }}^{m}(\mathcal{H})$ with coefficients $A_{(\alpha)} \in B(\mathcal{E}), \alpha \in \mathbb{F}_{n}^{+}$. That is

$$
F\left(X_{1}, \ldots, X_{n}\right)=\sum_{k=0}^{\infty} \sum_{|\alpha|=k} X_{\alpha} \otimes A_{(\alpha)},
$$

where the series converges in the operator norm topology for any $\left(X_{1}, \ldots, X_{n}\right) \in \mathbf{D}_{f, \text { rad }}^{m}(\mathcal{H})$. Let $0<r<1$ and assume that $F\left(r W_{1}, \ldots, r W_{n}\right)=0$. Taking into account relation (1.4), we have

$$
\left\langle F\left(r W_{1}, \ldots, r W_{n}\right)(1 \otimes x),\left(W_{\alpha} \otimes I_{\mathcal{E}}\right)(1 \otimes y)\right\rangle=\frac{r^{|\alpha|}}{b_{\alpha}^{(m)}}\left\langle A_{(\alpha)} x, y\right\rangle=0
$$

for any $x, y \in \mathcal{E}$ and $\alpha \in \mathbb{F}_{n}^{+}$. Therefore $A_{(\alpha)}=0$ for any $\alpha \in \mathbb{F}_{n}^{+}$, which proves our assertion. Due to this reason, throughout this paper, we assume that $\mathcal{H}$ is a separable infinite dimensional Hilbert space. 
Let $G\left(X_{1}, \ldots, X_{n}\right):=\sum_{k=0}^{\infty} \sum_{|\alpha|=k} X_{\alpha} \otimes C_{(\alpha)}$ be a free holomorphic function on $\mathbf{D}_{f, \text { rad }}^{m}(\mathcal{H})$ with coefficients in $B(\mathcal{E})$. Note that if $0<r_{1}<r_{2}<1$, then $\mathbf{D}_{f, r_{1}}^{m}(\mathcal{H}) \subseteq \mathbf{D}_{f, r_{2}}^{m}(\mathcal{H})$ and

$$
\left\|G_{r_{1}}\left(W_{1}, \ldots, W_{n}\right)\right\| \leq\left\|G_{r_{2}}\left(W_{1}, \ldots, W_{n}\right)\right\|
$$

where $G_{r}\left(W_{1}, \ldots, W_{n}\right):=\sum_{k=0}^{\infty} \sum_{|\alpha|=k}{ }^{|\alpha|} W_{\alpha} \otimes C_{(\alpha)}, 0 \leq r<1$, is convergent in the operator norm topology. Indeed, since $\varphi\left(W_{1}, \ldots, W_{n}\right):=\sum_{k=0}^{\infty} \sum_{|\alpha|=k} r_{2}^{|\alpha|} W_{\alpha} \otimes C_{(\alpha)}$ is in the tensor algebra $\mathcal{A}_{n}\left(\mathbf{D}_{f}^{m}\right) \bar{\otimes}_{\min } B(\mathcal{E})$ and $\left(r W_{1}, \ldots, r W_{n}\right) \in \mathbf{D}_{f}^{m}\left(F^{2}\left(H_{n}\right)\right)$, the noncommutative von Neumann inequality (1.7) implies

$$
\left\|\varphi\left(r W_{1}, \ldots, r W_{n}\right)\right\| \leq\left\|\varphi\left(W_{1}, \ldots, W_{n}\right)\right\|, \quad r \in[0,1) .
$$

Taking $r:=\frac{r_{1}}{r_{2}}$ in the latter inequality, our assertion follows.

We also remark that, for each $r \in[0,1)$, the map $G: \mathbf{D}_{f, r}^{m}(\mathcal{H}) \rightarrow B(\mathcal{H}) \otimes_{\min } B(\mathcal{E})$ defined by $G\left(X_{1}, \ldots, X_{n}\right):=\sum_{k=0}^{\infty} \sum_{|\alpha|=k} X_{\alpha} \otimes C_{(\alpha)}$ is continuous in the operator norm topology and

$$
\left\|G\left(X_{1}, \ldots, X_{n}\right)\right\| \leq\left\|G\left(r W_{1}, \ldots, r W_{n}\right)\right\|, \quad\left(X_{1}, \ldots, X_{n}\right) \in \mathbf{D}_{f, r}^{m}(\mathcal{H}) .
$$

Moreover, the series defining $G$ converges uniformly on $\mathbf{D}_{f, r}^{m}(\mathcal{H})$ in the same topology. Indeed, since $G_{r}\left(W_{1}, \ldots, W_{n}\right) \in \mathcal{A}_{n}\left(\mathbf{D}_{f}^{m}\right) \bar{\otimes}_{\min } B(\mathcal{E})$ and $\left(\frac{1}{r} X_{1}, \ldots, \frac{1}{r} X_{n}\right) \in \mathbf{D}_{f}^{\text {rad }}(\mathcal{H})$, and using again the noncommutative von Neumann inequality (1.7), we obtain

$$
\left\|G\left(X_{1}, \ldots, X_{n}\right)\right\|=\left\|G_{r}\left(\frac{1}{r} X_{1}, \ldots, \frac{1}{r} X_{n}\right)\right\| \leq\left\|G_{r}\left(W_{1}, \ldots, W_{n}\right)\right\|
$$

and

$$
\sum_{k=0}^{\infty}\left\|\sum_{|\alpha|=k} X_{\alpha} \otimes C_{(\alpha)}\right\| \leq \sum_{k=0}^{\infty}\left\|\sum_{|\alpha|=k} r^{|\alpha|} W_{\alpha} \otimes C_{(\alpha)}\right\|
$$

for any $\left(X_{1}, \ldots, X_{n}\right) \in \mathbf{D}_{f, r}^{m}(\mathcal{H})$. This clearly implies our assertion.

As in the particular case $m=1$ (see [29]), there is an important connection between the theory of free holomorphic functions on noncommutative domains $\mathbf{D}_{f, \text { rad }}^{m}, m \geq 1$, and the theory of holomorphic functions on domains in $\mathbb{C}^{d}([12])$.

Indeed, consider the case when $\mathcal{H}=\mathbb{C}^{p}$ and $p=1,2 \ldots$ Then $\mathbf{D}_{f}^{m}\left(\mathbb{C}^{p}\right)$ can be seen as a subset of $\mathbb{C}^{n p^{2}}$ with an arbitrary norm. We denote by $\operatorname{Int}\left(\mathbf{D}_{f}^{m}\left(\mathbb{C}^{p}\right)\right)$ the interior of the closed set $\mathbf{D}_{f}^{m}\left(\mathbb{C}^{p}\right)$. Note that $\operatorname{Int}\left(\mathbf{D}_{f}^{m}\left(\mathbb{C}^{p}\right)\right)$ are circular domains, i.e., $\left(e^{i \theta} \Lambda_{1}, \ldots, e^{i \theta} \Lambda_{n}\right) \in \operatorname{Int}\left(\mathbf{D}_{f}^{m}\left(\mathbb{C}^{p}\right)\right)$ for any $\left(\Lambda_{1}, \ldots \Lambda_{n}\right) \in$ $\operatorname{Int}\left(\mathbf{D}_{f}^{m}\left(\mathbb{C}^{p}\right)\right)$ and $\theta \in \mathbb{R}$. In the particular case when $p=1$, the interior $\operatorname{Int}\left(\mathbf{D}_{f}^{m}(\mathbb{C})\right)$ is a Reinhardt domain, i.e., $\left(e^{i \theta_{1}} \lambda_{1}, \ldots, e^{i \theta_{n}} \lambda_{n}\right) \in \operatorname{Int}\left(\mathbf{D}_{f}^{m}(\mathbb{C})\right)$ for any $\left(\lambda_{1}, \ldots \lambda_{n}\right) \in \operatorname{Int}\left(\mathbf{D}_{f}^{m}(\mathbb{C})\right)$ and $\theta_{1}, \ldots \theta_{n} \in \mathbb{R}$.

If $K$ is a compact subset in the interior of $\mathbf{D}_{f}^{m}\left(\mathbb{C}^{p}\right)$, then there exists $r \in(0,1)$ such that $K \subset \mathbf{D}_{f, r}^{m}\left(\mathbb{C}^{p}\right)$. Given a free holomorphic function on $\mathbf{D}_{f, \text { rad }}^{m}(\mathcal{H})$ with scalar coefficients, $F\left(X_{1}, \ldots, X_{n}\right):=\sum_{k=0}^{\infty} \sum_{|\alpha|=k} c_{\alpha} X_{\alpha}$, the results above show that

$$
\left\|F\left(\Lambda_{1}, \ldots, \Lambda_{n}\right)-\sum_{k=0}^{q} \sum_{|\alpha|=k} c_{\alpha} \Lambda_{\alpha}\right\| \leq \sum_{k=q+1}^{\infty}\left\|\sum_{|\alpha|=k} r^{|\alpha|} c_{\alpha} W_{\alpha}\right\|
$$

for any $\left(\Lambda_{1}, \ldots, \Lambda_{n}\right) \in K$. Consequently, $\sum_{k=0}^{q} \sum_{|\alpha|=k} c_{\alpha} \Lambda_{\alpha}$ converges to $F\left(\Lambda_{1}, \ldots, \Lambda_{n}\right)$ uniformly on $K$, as $q \rightarrow \infty$. Therefore, the map $\left(\Lambda_{1}, \ldots, \Lambda_{n}\right) \mapsto F\left(\Lambda_{1}, \ldots, \Lambda_{n}\right)$ is holomorphic on the interior of $\mathbf{D}_{f}^{m}\left(\mathbb{C}^{p}\right)$. To record this result, let $M_{p}$ denote the set of all $p \times p$ matrices with entries in $\mathbb{C}$.

Corollary 1.3. If $p \in \mathbb{N}$ and $F\left(X_{1}, \ldots, X_{n}\right):=\sum_{k=0}^{\infty} \sum_{|\alpha|=k} c_{\alpha} X_{\alpha}$ is a free holomorphic function on $\mathbf{D}_{f, \text { rad }}^{m}(\mathcal{H})$ with scalar coefficients, then its representation on $\mathbb{C}^{p}$, i.e., the map $F_{p}$ defined by

$$
\mathbb{C}^{n p^{2}} \supset \mathbf{D}_{f, \text { rad }}^{m}\left(\mathbb{C}^{p}\right) \ni\left(\Lambda_{1}, \ldots, \Lambda_{n}\right) \mapsto F\left(\Lambda_{1}, \ldots, \Lambda_{n}\right) \in M_{p} \subset \mathbb{C}^{p^{2}}
$$

is a holomorphic function on the interior of $\mathbf{D}_{f}^{m}\left(\mathbb{C}^{p}\right)$. 


\section{Noncommutative domain AlgEBras AND HaRdy AlgEBRAS}

We introduce the Hardy algebra $H^{\infty}\left(\mathbf{D}_{f, \text { rad }}^{m}\right)$ and the domain algebra $A\left(\mathbf{D}_{f, \text { rad }}^{m}\right)$ associated with the noncommutative domain $\mathbf{D}_{f, \text { rad }}^{m}, m \geq 1$. Using noncommutative Berezin transforms, we identify them with the noncommutative algebras $F_{n}^{\infty}\left(\mathbf{D}_{f}^{m}\right)$ and $\mathcal{A}_{n}\left(\mathbf{D}_{f}^{m}\right)$, respectively. Hardy algebras of bounded free holomorphic functions with operator-valued coefficients are also discussed.

Let us recall some definitions concerning completely bounded maps on operator spaces. We identify $M_{p}(B(\mathcal{H}))$, the set of $p \times p$ matrices with entries from $B(\mathcal{H})$, with $B\left(\mathcal{H}^{(p)}\right)$, where $\mathcal{H}^{(p)}$ is the direct sum of $p$ copies of $\mathcal{H}$. Thus we have a natural $C^{*}$-norm on $M_{p}(B(\mathcal{H}))$. If $X$ is an operator space, i.e., a closed subspace of $B(\mathcal{H})$, we consider $M_{p}(X)$ as a subspace of $M_{p}(B(\mathcal{H}))$ with the induced norm. Let $X, Y$ be operator spaces and $u: X \rightarrow Y$ be a linear map. Define the map $u_{p}: M_{p}(X) \rightarrow M_{p}(Y)$ by

$$
u_{p}\left(\left[x_{i j}\right]\right):=\left[u\left(x_{i j}\right)\right]
$$

We say that $u$ is completely bounded if $\|u\|_{c b}:=\sup _{p \geq 1}\left\|u_{p}\right\|<\infty$. If $\|u\|_{c b} \leq 1$ (resp. $u_{p}$ is an isometry for any $p \geq 1$ ) then $u$ is completely contractive (resp. isometric), and if $u_{p}$ is positive for all $p$, then $u$ is called completely positive. For more information on completely bounded maps, we refer to [16], [17, and $[8]$.

According to [24], if $T:=\left(T_{1}, \ldots, T_{n}\right) \in \mathbf{D}_{f}^{m}(\mathcal{H})$ is completely non-coisometric (c.n.c) with respect to $\mathbf{D}_{f}^{m}(\mathcal{H})$, i.e., there is no vector $h \in \mathcal{H}, h \neq 0$, such that

$$
\left\langle\Phi_{f, T}^{k}(I) h, h\right\rangle=\|h\|^{2} \quad \text { for any } k=1,2, \ldots,
$$

then

$$
\mathcal{B}_{T}(G):=\text { SOT }-\lim _{r \rightarrow 1} G_{r}\left(T_{1}, \ldots, T_{n}\right), \quad G=G\left(W_{1}, \ldots, W_{n}\right) \in F_{n}^{\infty}\left(\mathbf{D}_{f}^{m}\right),
$$

exists in the strong operator topology and defines a map $\mathcal{B}_{T}: F_{n}^{\infty}\left(\mathbf{D}_{f}^{m}\right) \rightarrow B(\mathcal{H})$, called the noncommutative Berezin transform at $T$ on the Hardy algebra $F_{n}^{\infty}\left(\mathbf{D}_{f}^{m}\right)$, with the following properties:

(i) $\mathcal{B}_{T}(G)=$ SOT- $\lim _{r \rightarrow 1} \mathbf{B}_{r T}[G]$, where $\mathbf{B}_{r T}$ is the noncommutative Berezin transform at $r T \in$ $\mathbf{D}_{f}^{m}(\mathcal{H})$

(ii) $\mathcal{B}_{T}$ is WOT-continuous (resp. SOT-continuous) on bounded sets;

(iii) $\mathcal{B}_{T}$ is a unital completely contractive homomorphism.

We remark that if $T$ is a pure $n$-tuple of operators, i.e., $\operatorname{SOT}-\lim _{p \rightarrow \infty} \Phi_{f, T}^{p}(I)=0$, then $T$ is c.n.c. and

$$
\mathcal{B}_{T}(G)=\widetilde{\mathbf{B}}_{T}[G]=K_{f, T}^{(m)^{*}}\left(G \otimes I_{\mathcal{D}_{f, m, T}}\right) K_{f, T}^{(m)}, \quad G \in F_{n}^{\infty}\left(\mathbf{D}_{f}^{m}\right),
$$

where $\mathcal{D}_{f, m, T}:=\overline{\Delta_{f, m, T}(\mathcal{H})}$ and $\widetilde{\mathbf{B}}_{T}$ is the extended noncommutative Berezin transform defined by relation (1.5). In particular, if $G\left(W_{1}, \ldots, W_{n}\right):=\sum_{\alpha \in \mathbb{F}_{n}^{+}} c_{\alpha} W_{\alpha}$ in the Hardy algebra $F_{n}^{\infty}\left(\mathbf{D}_{f}^{m}\right)$, then

$$
G\left(W_{1}, \ldots, W_{n}\right)=\text { SOT }-\lim _{r \rightarrow 1} G_{r}\left(W_{1}, \ldots, W_{n}\right),
$$

where $G_{r}\left(W_{1}, \ldots, W_{n}\right):=\mathbf{B}_{r W}[G]=\sum_{k=0}^{\infty} \sum_{|\alpha|=k} c_{\alpha} r^{|\alpha|} W_{\alpha}$, and

$$
\left\|G_{r}\left(W_{1}, \ldots, W_{n}\right)\right\| \leq\left\|G\left(W_{1}, \ldots, W_{n}\right)\right\|, \quad r \in[0,1) .
$$

Moreover, we can prove that

$$
\left\|G\left(W_{1}, \ldots, W_{n}\right)\right\|=\sup _{0 \leq r<1}\left\|G\left(r W_{1}, \ldots, r W_{n}\right)\right\|=\lim _{r \rightarrow 1}\left\|G\left(r W_{1}, \ldots, r W_{n}\right)\right\| .
$$

Indeed, if $\left.G\left(W_{1}, \ldots, W_{n}\right) \in F_{n}^{\infty}\left(\mathbf{D}_{f}^{m}\right)\right)$ and $\epsilon>0$, then there exists a polynomial $q \in F^{2}\left(H_{n}\right)$ with $\|q\|=1$ such that

$$
\left\|G\left(W_{1}, \ldots, W_{n}\right) q\right\|>\left\|G\left(W_{1}, \ldots, W_{n}\right)\right\|-\epsilon .
$$

By relation (2.2), there is $r_{0} \in(0,1)$ such that

$$
\left\|G_{r_{0}}\left(W_{1}, \ldots, W_{n}\right) q\right\|>\left\|G\left(W_{1}, \ldots, W_{n}\right)\right\|-\epsilon .
$$

Combining this with the inequality (2.3), we obtain

$$
\sup _{0 \leq r<1}\left\|G\left(r W_{1}, \ldots, r W_{n}\right)\right\|=\left\|G\left(W_{1}, \ldots, W_{n}\right)\right\| .
$$


Since the function $[0,1) \ni r \rightarrow\left\|G\left(r W_{1}, \ldots, r W_{n}\right)\right\| \in \mathbb{R}^{+}$is increasing (see relation (1.9)), we can complete the proof of our assertion.

Now, we are ready to introduce the noncommutative Hardy algebra $H^{\infty}\left(\mathbf{D}_{f, \text { rad }}^{m}\right)$. We denote by $\operatorname{Hol}\left(\mathbf{D}_{f, \text { rad }}^{m}\right)$ the set of all free holomorphic functions on $\mathbf{D}_{f, \text { rad }}^{m}$ with scalar coefficients. Due to Theorem 1.2. if $G \in \operatorname{Hol}\left(\mathbf{D}_{f, \text { rad }}^{m}\right)$, then $G\left(r W_{1}, \ldots, r W_{n}\right)$ is in the domain algebra $\mathcal{A}_{n}\left(\mathbf{D}_{f}^{m}\right)$ for any $r \in[0,1)$. Consequently, one can easily show that $\operatorname{Hol}\left(\mathbf{D}_{f, \text { rad }}^{m}\right)$ is an algebra. Let $H^{\infty}\left(\mathbf{D}_{f, \text { rad }}^{m}\right)$ denote the set of all elements $\varphi$ in $\operatorname{Hol}\left(\mathbf{D}_{f, \text { rad }}^{m}\right)$ such that

$$
\|\varphi\|_{\infty}:=\sup \left\|\varphi\left(X_{1}, \ldots, X_{n}\right)\right\|<\infty,
$$

where the supremum is taken over all $n$-tuples $\left(X_{1}, \ldots, X_{n}\right) \in \mathbf{D}_{f, \text { rad }}^{m}(\mathcal{H})$ and any Hilbert space $\mathcal{H}$. One can show that $H^{\infty}\left(\mathbf{D}_{f, \text { rad }}^{m}\right)$ is a Banach algebra under pointwise multiplication and the norm $\|\cdot\|_{\infty}$.

For each $p=1,2, \ldots$, we define the norms $\|\cdot\|_{p}: M_{p}\left(H^{\infty}\left(\mathbf{D}_{f, \text { rad }}^{m}\right)\right) \rightarrow[0, \infty)$ by setting

$$
\left\|\left[F_{i j}\right]_{p}\right\|_{p}:=\sup \left\|\left[F_{i j}\left(X_{1}, \ldots, X_{n}\right)\right]_{p}\right\|,
$$

where the supremum is taken over all $n$-tuples $\left(X_{1}, \ldots, X_{n}\right) \in \mathbf{D}_{f, \text { rad }}^{m}$ and any Hilbert space $\mathcal{H}$. It is easy to see that the norms $\|\cdot\|_{p}, p=1,2, \ldots$, determine an operator space structure on $H^{\infty}\left(\mathbf{D}_{f, \text { rad }}^{m}\right)$, in the sense of Ruan $([\underline{8}])$.

Given $g \in F_{n}^{\infty}\left(\mathbf{D}_{f}^{m}\right)$, the noncommutative Berezin transform associated with the noncommutative domain $\mathbf{D}_{f}^{m}$ generates a function

$$
\mathbf{B}[\varphi]: \mathbf{D}_{f, \text { rad }}^{m}(\mathcal{H}) \rightarrow B(\mathcal{H})
$$

by setting

$$
\mathbf{B}[\varphi]\left(X_{1}, \ldots, X_{n}\right):=\mathbf{B}_{X}[\varphi], \quad X:=\left(X_{1}, \ldots, X_{n}\right) \in \mathbf{D}_{f, \text { rad }}^{m}(\mathcal{H}) .
$$

We call $\mathbf{B}[\varphi]$ the Berezin transform of $\varphi$. In what follows, we identify the noncommutative algebra $F_{n}^{\infty}\left(\mathbf{D}_{f}^{m}\right)$ with the Hardy subalgebra $H^{\infty}\left(\mathbf{D}_{f, \text { rad }}^{m}\right)$ of bounded free holomorphic functions on $\mathbf{D}_{f \text {,rad }}^{m}$.

Theorem 2.1. The map $\Phi: H^{\infty}\left(\mathbf{D}_{f, \text { rad }}^{m}\right) \rightarrow F_{n}^{\infty}\left(\mathbf{D}_{f}^{m}\right)$ defined by

$$
\Phi\left(\sum_{\alpha \in \mathbb{F}_{n}^{+}} c_{\alpha} Z_{\alpha}\right):=\sum_{\alpha \in \mathbb{F}_{n}^{+}} c_{\alpha} W_{\alpha}
$$

is a completely isometric isomorphism of operator algebras. Moreover, if $G:=\sum_{\alpha \in \mathbb{F}_{n}^{+}} c_{\alpha} Z_{\alpha}$ is a free holomorphic function on the noncommutative domain $\mathbf{D}_{f, \text { rad }}^{m}$, then the following statements are equivalent:

(i) $G \in H^{\infty}\left(\mathbf{D}_{f, \text { rad }}^{m}\right)$;

(ii) $\sup _{0 \leq r<1}\left\|G\left(r W_{1}, \ldots, r W_{n}\right)\right\|<\infty$, where $G\left(r W_{1}, \ldots, r W_{n}\right):=\sum_{k=0}^{\infty} \sum_{|\alpha|=k} c_{\alpha} r^{|\alpha|} W_{\alpha}$;

(iii) there exists $\varphi \in F_{n}^{\infty}\left(\mathbf{D}_{f}^{m}\right)$ with $G=\mathbf{B}[\varphi]$.

In this case,

$$
\Phi(G)=\mathrm{SOT}-\lim _{r \rightarrow 1} G\left(r W_{1}, \ldots, r W_{n}\right) \quad \text { and } \quad \Phi^{-1}(\varphi)=\mathbf{B}[\varphi], \quad \varphi \in F_{n}^{\infty}\left(\mathbf{D}_{f}^{m}\right),
$$

where $\mathbf{B}$ is the noncommutative Berezin transform associated with the noncommutative domain $\mathbf{D}_{f}^{m}$.

Proof. First we need to show that the map $\Phi$ is well-defined. Let $G \in H^{\infty}\left(\mathbf{D}_{f, \text { rad }}^{m}\right)$ and let

$$
G\left(X_{1}, \ldots, X_{n}\right):=\sum_{k=0}^{\infty} \sum_{|\alpha|=k} c_{\alpha} X_{\alpha}, \quad\left(X_{1}, \ldots, X_{n}\right) \in \mathbf{D}_{f, \text { rad }}^{m}(\mathcal{H}),
$$

be its representation on a separable infinite dimensional Hilbert space $\mathcal{H}$. Since $\left(r W_{1}, \ldots, r W_{n}\right) \in$ $\mathbf{D}_{f, \text { rad }}^{m}\left(F^{2}\left(H_{n}\right)\right)$, we have

$$
\sup _{0 \leq r<1}\left\|G\left(r W_{1}, \ldots, r W_{n}\right)\right\| \leq\|G\|_{\infty}<\infty
$$


Hence, taking into account relation (1.4), we deduce that

$$
\begin{aligned}
\sum_{\alpha \in \mathbb{F}_{n}^{+}} r^{2|\alpha|}\left|c_{\alpha}\right|^{2} \frac{1}{b_{\alpha}^{(m)}} & =\left\|\sum_{\alpha \in \mathbb{F}_{n}^{+}} r^{|\alpha|} c_{\alpha} W_{\alpha}(1)\right\| \\
& \leq \sup _{0 \leq r<1}\left\|G\left(r W_{1}, \ldots, r W_{n}\right)\right\|<\infty
\end{aligned}
$$

for any $0 \leq r<1$. Consequently, $\sum_{\alpha \in \mathbb{F}_{n}^{+}}\left|c_{\alpha}\right|^{2} \frac{1}{b_{\alpha}^{(m)}}<\infty$, which shows that $G\left(W_{1}, \ldots, W_{n}\right) p$ is in the full Fock space $F^{2}\left(H_{n}\right)$ for any polynomial $p \in F^{2}\left(H_{n}\right)$. Now assume that $G\left(W_{1}, \ldots, W_{n}\right) \notin F_{n}^{\infty}\left(\mathbf{D}_{f}^{m}\right)$. According to the definition of $F_{n}^{\infty}\left(\mathbf{D}_{f}^{m}\right)$, for any fixed positive number $M$, there exists a polynomial $q \in F^{2}\left(H_{n}\right)$ with $\|q\|=1$ such that

$$
\left\|G\left(W_{1}, \ldots, W_{n}\right) q\right\|>M \text {. }
$$

Since $\left\|G_{r}\left(W_{1}, \ldots, W_{n}\right)(1)-G\left(W_{1}, \ldots, W_{n}\right)(1)\right\| \rightarrow 0$ as $r \rightarrow 1$, we have

$$
\left\|G\left(W_{1}, \ldots, W_{n}\right) q-G_{r}\left(W_{1}, \ldots, W_{n}\right) q\right\| \rightarrow 0, \quad \text { as } r \rightarrow 1 .
$$

Consequently, there is $r_{0} \in(0,1)$ such that $\left\|G_{r_{0}}\left(W_{1}, \ldots, W_{n}\right) q\right\|>M$, which implies $\left\|G_{r_{0}}\left(W_{1}, \ldots, W_{n}\right)\right\|>$ $M$. Since $M>0$ is arbitrary, we deduce that

$$
\sup _{0 \leq r<1}\left\|G\left(r W_{1}, \ldots, r W_{n}\right)\right\|=\infty
$$

which is a contradiction. Therefore, $G\left(W_{1}, \ldots, W_{n}\right) \in F_{n}^{\infty}\left(\mathbf{D}_{f}^{m}\right)$, which proves that the map $\Phi$ is welldefined. Moreover, due to relation (1.10), we have $\left\|G\left(X_{1}, \ldots, X_{n}\right)\right\| \leq\left\|G\left(r W_{1}, \ldots, r W_{n}\right)\right\|$ for any $\left(X_{1}, \ldots, X_{n}\right) \in \mathbf{D}_{f, r}^{m}(\mathcal{H})$. Using now relation (2.4), we deduce that

$$
\left\|G\left(W_{1}, \ldots, W_{n}\right)\right\|=\sup _{0 \leq r<1}\left\|G\left(r W_{1}, \ldots, r W_{n}\right)\right\|=\|G\|_{\infty} .
$$

Therefore, $\Phi$ is a well-defined isometric linear map. Moreover, relation (2.2), implies

$$
\Phi(G)=G\left(W_{1}, \ldots, G_{m}\right)=\mathrm{SOT}-\lim _{r \rightarrow 1} G_{r}\left(W_{1}, \ldots, W_{n}\right) .
$$

We show now that $\Phi$ is a surjective map. To this end, let $\varphi:=\sum_{\alpha \in \mathbb{F}_{n}^{+}} c_{\alpha} W_{\alpha}$ be in $F_{n}^{\infty}\left(\mathbf{D}_{f}^{m}\right)$. Then $\sum_{\alpha \in \mathbb{F}_{n}^{+}}\left|c_{\alpha}\right|^{2} \frac{1}{b_{\alpha}^{(m)}}<\infty$, which implies

$$
\limsup _{k \rightarrow \infty}\left(\sum_{|\alpha|=k}\left|c_{\alpha}\right|^{2} \frac{1}{b_{\alpha}^{(m)}}\right)^{\frac{1}{2 k}} \leq 1 .
$$

Using now Theorem 1.2, we deduce that $G\left(Z_{1}, \ldots, Z_{n}\right):=\sum_{\alpha \in \mathbb{F}_{n}^{+}} c_{\alpha} Z_{\alpha}$ is a free holomorphic function on the noncommutative domain $\mathbf{D}_{f, \text { rad }}^{m}$. Due to inequalities (1.11), and (2.3), we have

$$
\left\|G\left(X_{1}, \ldots, X_{n}\right)\right\| \leq\left\|G\left(r W_{1}, \ldots, r W_{n}\right)\right\| \leq\left\|G\left(W_{1}, \ldots, W_{n}\right)\right\|
$$

for any $\left(X_{1}, \ldots, X_{n}\right) \in \mathbf{D}_{f, r}^{m}(\mathcal{H})$ and $r \in[0,1)$. Hence, we deduce that

$$
\sup _{\left(X_{1}, \ldots, X_{n}\right) \in \mathbf{D}_{f, \text { rad }}^{m}(\mathcal{H})}\left\|G\left(X_{1}, \ldots, X_{n}\right)\right\| \leq\left\|G\left(W_{1}, \ldots, W_{n}\right)\right\|<\infty
$$

which proves that $G \in H^{\infty}\left(\mathbf{D}_{f, \text { rad }}^{m}\right)$. This shows that the map $\Phi$ is surjective. Therefore, we have proved that $\Phi$ is an isometric isomorphism of operator algebras. Using the same techniques and passing to matrices, one can prove that $\Phi$ is a completely isometric isomorphism.

Moreover, note that if $X:=\left(X_{1}, \ldots, X_{n}\right) \in \mathbf{D}_{f, \text { rad }}^{m}$, then $G\left(X_{1}, \ldots, X_{n}\right)=\sum_{k=0}^{\infty} \sum_{|\alpha|=k} c_{\alpha} X_{\alpha}$ is convergent in the operator norm topology. Due to the properties of the noncommutative Berezin transform, 
for any $r \in[0,1)$, we have

$$
\begin{aligned}
\sum_{k=0}^{\infty} \sum_{|\alpha|=k} c_{\alpha} r^{|\alpha|} X_{\alpha} & =\sum_{k=0}^{\infty} \mathbf{B}_{X}\left[\sum_{|\alpha|=k} c_{\alpha} r^{|\alpha|} W_{\alpha}\right] \\
& =\mathbf{B}_{X}\left[\sum_{k=0}^{\infty} \sum_{|\alpha|=k} c_{\alpha} r^{|\alpha|} W_{\alpha}\right] \\
& =\mathbf{B}_{X}\left[\varphi_{r}\right]=K_{X, f}^{(m)^{*}}\left[\varphi_{r} \otimes I_{\mathcal{H}}\right] K_{X, f}^{(m)} .
\end{aligned}
$$

Recall that $\left\|\varphi_{r}\right\| \leq\|\varphi\|$ for any $r \in[0,1)$ and $\varphi=$ SOT- $\lim _{r \rightarrow 1} \varphi_{r}$. Passing to the limit as $r \rightarrow 1$ in the equalities above, and using the continuity of the free holomorphic function $G$ on $\mathbf{D}_{f \text {,rad }}^{m}$, we obtain

$$
G\left(X_{1}, \ldots, X_{n}\right)=\mathbf{B}_{X}[\varphi], \quad X:=\left(X_{1}, \ldots, X_{n}\right) \in \mathbf{D}_{f, \mathrm{rad}}^{m} .
$$

The equivalences mentioned in the theorem can be easily deduced from the considerations above and the properties of the noncommutative Berezin transform. The proof is complete.

We denote by $A\left(\mathbf{D}_{f, \text { rad }}^{m}\right)$ the set of all elements $G$ in $\operatorname{Hol}\left(\mathbf{D}_{f, \text { rad }}^{m}\right)$ such that the mapping

$$
\mathbf{D}_{f, \operatorname{rad}}^{m}(\mathcal{H}) \ni\left(X_{1}, \ldots, X_{n}\right) \mapsto G\left(X_{1}, \ldots, X_{n}\right) \in B(\mathcal{H})
$$

has a continuous extension to $\left[\mathbf{D}_{f, \text { rad }}^{m}(\mathcal{H})\right]^{-}=\mathbf{D}_{f}^{m}(\mathcal{H})$ for any Hilbert space $\mathcal{H}$. One can show that $A\left(\mathbf{D}_{f, \text { rad }}^{m}\right)$ is a Banach algebra under pointwise multiplication and the norm $\|\cdot\|_{\infty}$. Moreover, we can identify the domain algebra $\mathcal{A}_{n}\left(\mathbf{D}_{f}^{m}\right)$ with the subalgebra $A\left(\mathbf{D}_{f, \text { rad }}^{m}\right)$.

Theorem 2.2. The map $\Phi: A\left(\mathbf{D}_{f, \text { rad }}^{m}\right) \rightarrow \mathcal{A}_{n}\left(\mathbf{D}_{f}^{m}\right)$ defined by

$$
\Phi\left(\sum_{\alpha \in \mathbb{F}_{n}^{+}} c_{\alpha} Z_{\alpha}\right):=\sum_{\alpha \in \mathbb{F}_{n}^{+}} c_{\alpha} W_{\alpha}
$$

is a completely isometric isomorphism of operator algebras. Moreover, if $G:=\sum_{\alpha \in \mathbb{F}_{n}^{+}} c_{\alpha} Z_{\alpha}$ is a free holomorphic function on the domain $\mathbf{D}_{f, \mathrm{rad}}^{m}$, then the following statements are equivalent:

(i) $G \in A\left(\mathbf{D}_{f, \text { rad }}^{m}\right)$;

(ii) $G\left(r W_{1}, \ldots, r W_{n}\right):=\sum_{k=0}^{\infty} \sum_{|\alpha|=k} c_{\alpha} r^{|\alpha|} W_{\alpha}$ is convergent in the operator norm topology as $r \rightarrow 1$;

(iii) there exists $\varphi \in \mathcal{A}_{n}\left(\mathbf{D}_{f}^{m}\right)$ with $G=\mathbf{B}[\varphi]$.

In this case,

$$
\Phi(G)=\lim _{r \rightarrow 1} G\left(r W_{1}, \ldots, r W_{n}\right) \quad \text { and } \quad \Phi^{-1}(\varphi)=\mathbf{B}[\varphi], \quad \varphi \in \mathcal{A}_{n}\left(\mathbf{D}_{f}^{m}\right),
$$

where $\mathbf{B}$ is the noncommutative Berezin transform associated with the noncommutative domain $\mathbf{D}_{f}^{m}$.

Proof. Assume that $G \in A\left(\mathbf{D}_{f, \text { rad }}^{m}\right)$. Since $\left(r W_{1}, \ldots, r W_{n}\right) \in \mathbf{D}_{f, \text { rad }}^{m}\left(F^{2}\left(H_{n}\right)\right)$ for $r \in[0,1)$, we deduce that $\lim _{r \rightarrow \infty} G\left(r W_{1}, \ldots, r W_{n}\right)$ exists in the operator norm topology. Using the fact that $G\left(r W_{1}, \ldots, r W_{n}\right)$ is in the domain algebra $\mathcal{A}_{n}\left(\mathbf{D}_{f}^{m}\right)$, which is closed in the operator norm topology, we have

$$
\varphi:=\lim _{r \rightarrow \infty} G\left(r W_{1}, \ldots, r W_{n}\right) \in \mathcal{A}_{n}\left(\mathbf{D}_{f}^{m}\right) .
$$

On the other hand, due to Theorem 2.1, we deduce that $\Phi(G):=\sum_{\alpha \in \mathbb{F}_{n}} c_{\alpha} W_{\alpha}$ is in $F_{n}^{\infty}\left(\mathcal{D}_{f}\right)$ and

$$
\Phi(G)=\text { SOT }-\lim _{r \rightarrow 1} G\left(r W_{1}, \ldots, r W_{n}\right) .
$$

Therefore, we have

$$
\Phi(G)=\varphi=\lim _{r \rightarrow 1} G\left(r W_{1}, \ldots, r W_{n}\right) \in \mathcal{A}_{n}\left(\mathbf{D}_{f}^{m}\right)
$$


and

$$
G\left(X_{1}, \ldots, X_{n}\right)=\lim _{r \rightarrow 1} \mathbf{B}_{X}\left[G\left(r W_{1}, \ldots, r W_{n}\right)\right]=\mathbf{B}_{X}[\varphi], \quad X:=\left(X_{1}, \ldots, X_{n}\right) \in \mathbf{D}_{f, \operatorname{rad}}^{m}(\mathcal{H}) .
$$

Here we used the continuity of the Berezin transform in the operator norm topology.

Now, let $\varphi \in \mathcal{A}_{n}\left(\mathbf{D}_{f}^{m}\right) \subset F_{n}^{\infty}\left(\mathbf{D}_{f}^{m}\right)$ have the Fourier representation $\varphi=\sum_{\alpha \in \mathbb{F}_{n}^{+}} c_{\alpha} W_{\alpha}$. Then, for any $n$-tuple $\left(Y_{1}, \ldots, Y_{n}\right) \in \mathbf{D}_{f}^{m}(\mathcal{H})$,

$$
\tilde{G}\left(Y_{1}, \ldots, Y_{n}\right):=\lim _{r \rightarrow 1} \mathbf{B}_{r Y}[\varphi]=\lim _{r \rightarrow 1} \varphi\left(r Y_{1}, \ldots, r Y_{n}\right)
$$

exists in the operator norm and

$$
\left\|\tilde{G}\left(Y_{1}, \ldots, Y_{n}\right)\right\| \leq\|\varphi\| \quad \text { for any }\left(Y_{1}, \ldots, Y_{n}\right) \in \mathbf{D}_{f}^{m}(\mathcal{H}) .
$$

Note also that $\tilde{G}$ is an extension of the free holomorphic function

$$
G\left(X_{1}, \ldots, X_{n}\right)=\mathbf{B}_{X}[\varphi]=\sum_{k=0}^{\infty} \sum_{|\alpha|=k} c_{\alpha} X_{\alpha}, \quad\left(X_{1}, \ldots, X_{n}\right) \in \mathbf{D}_{f, \text { rad }}^{m}(\mathcal{H}) .
$$

Indeed, if $\left(X_{1}, \ldots, X_{n}\right) \in \mathbf{D}_{f, \text { rad }}^{m}(\mathcal{H})$, then

$$
\begin{aligned}
\tilde{G}\left(X_{1}, \ldots, X_{n}\right) & =\lim _{r \rightarrow 1} \varphi\left(r X_{1}, \ldots, r X_{n}\right) \\
& =\lim _{r \rightarrow 1} G\left(r X_{1}, \ldots, r X_{n}\right)=G\left(X_{1}, \ldots, X_{n}\right) .
\end{aligned}
$$

The last equality is due to the fact that $G$ is continuous on $\mathbf{D}_{f \text {,rad }}^{m}(\mathcal{H})$.

Let us prove that $\tilde{G}: \mathbf{D}_{f}^{m}(\mathcal{H}) \rightarrow B(\mathcal{H})$ is a continuous map. Since $\varphi \in \mathcal{A}_{n}\left(\mathbf{D}_{f}^{m}\right)$, for any $\epsilon>0$ there exists $r_{0} \in[0,1)$ such that $\left\|\varphi-\varphi\left(r_{0} W_{1}, \ldots, r_{0} W_{n}\right)\right\|<\epsilon$. Applying the noncommutative von Neumann inequality to $\varphi-\varphi_{r_{0}} \in \mathcal{A}_{n}\left(\mathbf{D}_{f}^{m}\right)$ and using relation (2.5), we deduce that

$$
\left\|\tilde{G}\left(T_{1}, \ldots, T_{n}\right)-\varphi_{r_{0}}\left(T_{1}, \ldots, T_{n}\right)\right\| \leq\left\|\varphi-\varphi_{r_{0}}\right\|<\frac{\epsilon}{3}
$$

for any $\left(T_{1}, \ldots, T_{n}\right) \in \mathbf{D}_{f}^{m}(\mathcal{H})$. We recall that $G$ is a continuous function on $\mathbf{D}_{f, \text { rad }}^{m}(\mathcal{H})$. Therefore, there exists $\delta>0$ such that

$$
\left\|G_{r_{0}}\left(T_{1}, \ldots, T_{n}\right)-G_{r_{0}}\left(Y_{1}, \ldots, Y_{n}\right)\right\|<\frac{\epsilon}{3}
$$

for any $n$-tuple $\left(Y_{1}, \ldots, Y_{n}\right)$ in $\mathbf{D}_{f}^{m}(\mathcal{H})$ such that $\left\|\left(T_{1}-Y_{1}, \ldots, T_{n}-Y_{n}\right)\right\|<\delta$. Hence, and using relation (2.6), we have

$$
\begin{aligned}
\left\|\tilde{G}\left(T_{1}, \ldots, T_{n}\right)-\tilde{G}\left(Y_{1}, \ldots, Y_{n}\right)\right\| \leq \| \tilde{G} & \left(T_{1}, \ldots, T_{n}\right)-\varphi_{r_{0}}\left(T_{1}, \ldots, T_{n}\right) \| \\
& +\left\|G_{r_{0}}\left(T_{1}, \ldots, T_{n}\right)-G_{r_{0}}\left(Y_{1}, \ldots, Y_{n}\right)\right\| \\
& +\left\|\varphi_{r_{0}}\left(Y_{1}, \ldots, Y_{n}\right)-\tilde{G}\left(Y_{1}, \ldots, Y_{n}\right)\right\|<\epsilon,
\end{aligned}
$$

whenever $\left\|\left(T_{1}-Y_{1}, \ldots, T_{n}-Y_{n}\right)\right\|<\delta$. This proves the continuity of $\tilde{G}$ on $\mathbf{D}_{f}^{m}(\mathcal{H})$. Therefore, $G \in A\left(\mathbf{D}_{f}^{m}\right)$ and, moreover, we have

$$
\Phi(G)=\varphi \quad \text { and } \quad \Phi^{-1}(\varphi)=\mathbf{B}[\varphi], \quad \varphi \in \mathcal{A}_{n}\left(\mathbf{D}_{f}^{m}\right)
$$

Using now Theorem 2.1] we deduce that he map $\Phi: A\left(\mathbf{D}_{f, \text { rad }}^{m}\right) \rightarrow \mathcal{A}_{n}\left(\mathbf{D}_{f}^{m}\right)$ is a completely isometric isomorphism of operator algebras. The equivalences mentioned in the theorem can be easily deduced from the considerations above and the properties of the noncommutative Berezin transform. This completes the proof.

Due to Theorem 2.2 and Corollary [1.3, we deduce the following result.

Corollary 2.3. If $p \in \mathbb{N}$ and $F\left(X_{1}, \ldots, X_{n}\right):=\sum_{k=0}^{\infty} \sum_{|\alpha|=k} c_{\alpha} X_{\alpha}$ is in $A\left(\mathbf{D}_{f, \text { rad }}^{m}\right)$, then its representation on $\mathbb{C}^{p}$, i.e., the map $F_{p}$ defined by

$$
\mathbb{C}^{n p^{2}} \supset \mathbf{D}_{f}^{m}\left(\mathbb{C}^{p}\right) \ni\left(\Lambda_{1}, \ldots, \Lambda_{n}\right) \mapsto F\left(\Lambda_{1}, \ldots, \Lambda_{n}\right) \in M_{p} \subset \mathbb{C}^{p^{2}}
$$

is a continuous map on $\mathbf{D}_{f}^{m}\left(\mathbb{C}^{p}\right)$ and holomorphic on the interior of $\mathbf{D}_{f}^{m}\left(\mathbb{C}^{p}\right)$. 
We remark that one can obtain operator-valued versions of Theorem 2.1 and Theorem 2.2, which we summarize in what follows. Let $\mathcal{E}$ be a separable Hilbert space. We denote by $H_{\mathcal{E}}\left(\mathbf{D}_{f, \text { rad }}^{m}\right)$ the set of all free holomorphic functions on the noncommutative ball $\mathbf{D}_{f \text {,rad }}^{m}$ and coefficients in $B(\mathcal{E})$. Let $H_{\mathcal{E}}^{\infty}\left(\mathbf{D}_{f, \text { rad }}^{m}\right)$ denote the set of all elements $F$ in $\operatorname{Hol}_{\mathcal{E}}\left(\mathbf{D}_{f, \text { rad }}^{m}\right)$ such that

$$
\|F\|_{\infty}:=\sup \left\|F\left(X_{1}, \ldots, X_{n}\right)\right\|<\infty
$$

where the supremum is taken over all $n$-tuples of operators $\left(X_{1}, \ldots, X_{n}\right) \in \mathbf{D}_{f \text {,rad }}^{m}(\mathcal{H})$ and any Hilbert space $\mathcal{H}$. The noncommutative Hardy space $H_{\mathcal{E}}^{\infty}\left(\mathbf{D}_{f, \text { rad }}^{m}\right)$ can be identified to $F_{n}^{\infty}\left(\mathbf{D}_{f}^{m}\right) \bar{\otimes} B(\mathcal{E})$, the weakly closed operator algebra generated by the spatial tensor product. More precisely, a bounded free holomorphic function $F$ on $\mathbf{D}_{f \text {,rad }}^{m}$ with coefficients in $B(\mathcal{E})$ is uniquely determined by its model boundary function $\widetilde{F}\left(W_{1}, \ldots, W_{n}\right) \in F_{n}^{\infty}\left(\mathbf{D}_{f}^{m}\right) \bar{\otimes} B(\mathcal{E})$ defined by

$$
\widetilde{F}=\widetilde{F}\left(W_{1}, \ldots, W_{n}\right):=\text { SOT }-\lim _{r \rightarrow 1} F\left(r W_{1}, \ldots, r W_{n}\right) .
$$

Moreover, $F$ is the noncommutative Berezin transform of $\widetilde{F}\left(W_{1}, \ldots, W_{n}\right)$ at $X:=\left(X_{1}, \ldots, X_{n}\right) \in$ $\mathbf{D}_{f, \text { rad }}^{m}(\mathcal{H})$, i.e.,

$$
F\left(X_{1}, \ldots, X_{n}\right)=\left(\mathbf{B}_{X} \otimes I_{\mathcal{E}}\right)\left[\widetilde{F}\left(W_{1}, \ldots, W_{n}\right)\right] .
$$

We denote by $A_{\mathcal{E}}\left(\mathbf{D}_{f, \text { rad }}^{m}\right)$ the set of all elements $\varphi$ in $H o l_{\mathcal{E}}\left(\mathbf{D}_{f, \text { rad }}^{m}\right)$ such that the mapping

$$
\mathbf{D}_{f, \text { rad }}^{m}(\mathcal{H}) \ni\left(X_{1}, \ldots, X_{n}\right) \mapsto \varphi\left(X_{1}, \ldots, X_{n}\right) \in B(\mathcal{H})
$$

has a continuous extension to $\left[\mathbf{D}_{f, \text { rad }}^{m}(\mathcal{H})\right]^{-}=\mathbf{D}_{f}^{m}(\mathcal{H})$ for any Hilbert space $\mathcal{H}$. One can show that $A_{\mathcal{E}}\left(\mathbf{D}_{f, \text { rad }}^{m}\right)$ is a Banach algebra under pointwise multiplication and the norm $\|\cdot\|_{\infty}$. Moreover, we can identify the tensor algebra $\mathcal{A}_{n}\left(\mathbf{D}_{f}^{m}\right) \bar{\otimes}_{\min } B(\mathcal{E})$ (the closed operator algebra generated by the spatial tensor product) with the subalgebra $A_{\mathcal{E}}\left(\mathbf{D}_{f, \text { rad }}^{m}\right)$. Since the proofs of these results are very similar to those of Theorem 2.1 and Theorem 2.2, we shall omit them.

\section{COMPOSITIONS OF FREE HOLOMORPHIC FUNCTIONS}

In this section we present several results concerning the composition of free holomorphic functions on noncommutative domains $\mathbf{D}_{f, \text { rad }}^{m}$. These results are used, throughout this paper, to study free biholomorphic functions.

Let $f$ be a positive regular free holomorphic function with $n$ indeterminates and consider a free holomorphic function $\Phi: \mathbf{D}_{f, \text { rad }}^{m}(\mathcal{H}) \rightarrow\left[B(\mathcal{H}) \bar{\otimes}_{\min } B(\mathcal{Y})\right]^{p}$. Then we have $\Phi=\left(\Phi_{1}, \ldots, \Phi_{p}\right)$, where each mapping $\Phi_{j}: \mathbf{D}_{f, \text { rad }}^{m}(\mathcal{H}) \rightarrow B(\mathcal{H}) \bar{\otimes}_{\min } B(\mathcal{Y})$ is a free holomorphic function with representation

$$
\Phi_{j}(X)=\sum_{k=0}^{\infty} \sum_{\alpha \in \mathbb{F}_{n}^{+},|\alpha|=k} X_{\alpha} \otimes B_{(\alpha)}^{(j)}, \quad X:=\left(X_{1}, \ldots, X_{n}\right) \in \mathbf{D}_{f, \mathrm{rad}}^{m}(\mathcal{H}),
$$

for some $B_{(\alpha)}^{(j)} \in B(\mathcal{Y}), \alpha \in \mathbb{F}_{n}^{+}$. Assume now that

$$
\Phi(X) \in \mathbf{D}_{g, \text { rad }}^{l}(\mathcal{H} \otimes \mathcal{Y}) \quad \text { for any } X \in \mathbf{D}_{f, \text { rad }}^{m}(\mathcal{H}),
$$

where $g$ is a positive regular free holomorphic function with $p$ indeterminates, and $l \geq 1$. Consider a free holomorphic function $F: \mathbf{D}_{g \text {,rad }}^{l}(\mathcal{K}) \rightarrow B(\mathcal{K}) \bar{\otimes}_{\text {min }} B(\mathcal{E})$ with standard representation

$$
F\left(Y_{1}, \ldots, Y_{p}\right):=\sum_{k=0}^{\infty} \sum_{\alpha \in \mathbb{F}_{p}^{+},|\alpha|=k} Y_{\alpha} \otimes A_{(\alpha)}, \quad\left(Y_{1}, \ldots, Y_{p}\right) \in \mathbf{D}_{g, \text { rad }}^{l}(\mathcal{K})
$$

for some bounded operators $A_{(\alpha)} \in B(\mathcal{E}), \alpha \in \mathbb{F}_{p}^{+}$. Note that it makes sense to define the mapping $F \circ \Phi: \mathbf{D}_{f, \text { rad }}^{m}(\mathcal{H}) \rightarrow B(\mathcal{H}) \bar{\otimes}_{\min } B(\mathcal{Y}) \bar{\otimes}_{\min } B(\mathcal{E})$ by setting

$$
(F \circ \Phi)\left(X_{1}, \ldots, X_{n}\right):=\sum_{k=0}^{\infty} \sum_{\alpha \in \mathbb{F}_{p}^{+},|\alpha|=k} \Phi_{\alpha}\left(X_{1}, \ldots, X_{n}\right) \otimes A_{(\alpha)}, \quad\left(X_{1}, \ldots, X_{n}\right) \in \mathbf{D}_{f, \text { rad }}^{m}(\mathcal{H}),
$$


where the convergence is in the operator norm topology. In what follows, we prove that $F \circ \Phi$ is a free holomorphic function on $\mathbf{D}_{f, \text { rad }}^{m}(\mathcal{H})$ with standard representation

$$
(F \circ \Phi)\left(X_{1}, \ldots, X_{n}\right)=\sum_{k=0}^{\infty} \sum_{\sigma \in \mathbb{F}_{n}^{+},|\sigma|=k} X_{\sigma} \otimes C_{(\sigma)}, \quad\left(X_{1}, \ldots, X_{n}\right) \in \mathbf{D}_{f, \text { rad }}^{m}(\mathcal{H}),
$$

where $C_{(\sigma)} \in B(\mathcal{Y} \otimes \mathcal{E})$ is defined by

$$
\left\langle C_{(\sigma)} x, y\right\rangle=\frac{b_{\sigma}^{(m)}}{r^{|\sigma|}}\left\langle\left(W_{\sigma}^{*} \otimes I_{\mathcal{Y} \otimes \mathcal{E}}\right)(F \circ \Phi)\left(r W_{1}, \ldots, r W_{n}\right)(1 \otimes x), 1 \otimes y\right\rangle
$$

for any $\sigma \in \mathbb{F}_{n}^{+}$and $x, y \in \mathcal{Y} \otimes \mathcal{E}$.

First, we need the following result.

Lemma 3.1. Let $f$ and $g$ be positive regular free holomorphic functions with $n$ and $p$ indeterminates, respectively, and let $m, l \geq 1$. If $\Phi: \mathbf{D}_{f, \text { rad }}^{m}(\mathcal{H}) \rightarrow\left[B(\mathcal{H}) \bar{\otimes}_{\min } B(\mathcal{Y})\right]^{p}$ is a free holomorphic function, then range $\Phi \subseteq \mathbf{D}_{g, \text { rad }}^{l}(\mathcal{H} \otimes \mathcal{Y})$ if and only if

$$
\Phi\left(r W_{1}, \ldots, r W_{n}\right) \in \mathbf{D}_{g, \text { rad }}^{l}\left(F^{2}\left(H_{n}\right) \otimes \mathcal{Y}\right) \quad \text { for any } r \in[0,1),
$$

where $\left(W_{1}, \ldots, W_{n}\right)$ is the universal model associated with the noncommutative domain $\mathbf{D}_{f}^{m}$.

Proof. Since $\mathcal{H}$ is a separable infinite dimensional Hilbert space and $\left(r W_{1}, \ldots, r W_{n}\right) \in \mathbf{D}_{f, \text { rad }}^{m}\left(F^{2}\left(H_{n}\right)\right)$ the direct implication is obvious. To prove the converse, assume that $\Phi=\left(\Phi_{1}, \ldots, \Phi_{p}\right)$ is a free holomorphic function on $\mathbf{D}_{f, \text { rad }}^{m}(\mathcal{H})$ and $\Phi\left(r W_{1}, \ldots, r W_{n}\right) \in \mathbf{D}_{g, \text { rad }}^{l}\left(F^{2}\left(H_{n}\right) \otimes \mathcal{Y}\right)$ for any $r \in(0,1)$. Let $X:=\left(X_{1}, \ldots, X_{n}\right)$ be in $\mathbf{D}_{f, \text { rad }}^{m}(\mathcal{H})$. Then there exists $\gamma \in(0,1)$ such that $X \in \mathbf{D}_{f, \gamma}^{m}(\mathcal{H})$. Since $\Phi$ is a free holomorphic function on $\mathbf{D}_{f, \text { rad }}^{m}(\mathcal{H})$, for each $j=1, \ldots, p$, the operator $\Phi_{j}\left(\gamma W_{1}, \ldots, \gamma W_{n}\right)$ is in $\mathcal{A}_{n}\left(\mathbf{D}_{f}^{m}\right) \bar{\otimes}_{\min } B(\mathcal{Y})$ and there is $s \in(0,1)$ such that

$$
\frac{1}{s} \Phi(\gamma W):=\left(\frac{1}{s} \Phi_{1}\left(\gamma W_{1}, \ldots, \gamma W_{n}\right), \ldots, \frac{1}{s} \Phi_{p}\left(\gamma W_{1}, \ldots, \gamma W_{n}\right)\right) \in \mathbf{D}_{g}^{l}\left(F^{2}\left(H_{n}\right) \otimes \mathcal{Y}\right) .
$$

This implies $\left(i d-\Phi_{g, \frac{1}{s}} \Phi(\gamma W)\right)^{k}(I) \geq 0$ for $1 \leq k \leq l$. Since $\Phi=\left(\Phi_{1}, \ldots, \Phi_{p}\right)$ is a free holomorphic function on $\mathbf{D}_{f, \text { rad }}^{m}(\mathcal{H})$, each $\Phi_{j}\left(X_{1}, \ldots, X_{n}\right)$ is given by a series which is convergent in the operator topology and so is $\Phi_{j}(\gamma W) \in \mathcal{A}_{n}\left(\mathbf{D}_{f}^{m}\right)$. Therefore, using the properties of the noncommutative Berezin transform, we have

$$
\Phi_{\alpha}(X) \Phi_{\alpha}(X)^{*}=\mathbf{B}_{\frac{1}{\gamma} X}\left[\Phi_{\alpha}(\gamma W) \Phi_{\alpha}(\gamma W)^{*}\right]
$$

for any $X \in \mathbf{D}_{f, \gamma}^{m}(\mathcal{H})$ and $\alpha \in \mathbb{F}_{n}^{+}$. Since, due to (3.1), $\left(i d-\Phi_{g, \frac{1}{s} \Phi(\gamma W)}\right)^{k}(I) \leq I$ for $1 \leq k \leq l$, and using the fact that the noncommutative Berezin transform is a completely positive map which is SOT-continuous on bounded sets, we deduce that

$$
\left(i d-\Phi_{g, \frac{1}{s} \Phi(X)}\right)^{k}(I)=\mathbf{B}_{\frac{1}{\gamma} X}\left[\left(i d-\Phi_{g, \frac{1}{s} \Phi(\gamma W)}\right)^{k}(I)\right] \geq 0, \quad 1 \leq k \leq l .
$$

Hence, we infer that $\left(\frac{1}{s} \varphi_{1}\left(X_{1}, \ldots, X_{n}\right), \ldots, \frac{1}{s} \varphi_{p}\left(X_{1}, \ldots, X_{n}\right)\right) \in \mathbf{D}_{g}^{l}(\mathcal{H} \otimes \mathcal{Y})$, which shows that the $n$-tuple $\left(\varphi_{1}\left(X_{1}, \ldots, X_{n}\right), \ldots, \varphi_{p}\left(X_{1}, \ldots, X_{n}\right)\right)$ is in $\mathbf{D}_{g, \text { rad }}^{l}(\mathcal{H} \otimes \mathcal{Y})$. This completes the proof.

The next result shows that the composition of free holomorphic functions with operator-valued coefficients is a free holomorphic function.

Theorem 3.2. Let $f$ and $g$ be positive regular free holomorphic functions with $n$ and $p$ indeterminates, respectively, and let $m, l \geq 1$. Let $F: \mathbf{D}_{g, \text { rad }}^{l}(\mathcal{K}) \rightarrow B(\mathcal{K}) \bar{\otimes} B(\mathcal{E})$ and $\Phi: \mathbf{D}_{f, \text { rad }}^{m}(\mathcal{H}) \rightarrow\left[B(\mathcal{H}) \bar{\otimes}_{\text {min }} B(\mathcal{Y})\right]^{p}$ be free holomorphic functions such that range $\Phi \subseteq \mathbf{D}_{g \text {,rad }}^{l}(\mathcal{H} \otimes \mathcal{Y})$. Then $F \circ \Phi$ is a free holomorphic function on $\mathbf{D}_{f, \text { rad }}^{m}(\mathcal{H})$. If, in addition, $F$ is bounded, then $F \circ \Phi$ is bounded and $\|F \circ \Phi\|_{\infty} \leq\|F\|_{\infty}$. 
Proof. Suppose that $F$ has the standard representation

$$
F\left(Y_{1}, \ldots, Y_{p}\right)=\sum_{k=0}^{\infty} \sum_{\beta \in \mathbb{F}_{p}^{+},|\beta|=k} Y_{\alpha} \otimes A_{(\beta)}, \quad\left(Y_{1}, \ldots, Y_{p}\right) \in \mathbf{D}_{g, \mathrm{rad}}^{l}(\mathcal{K}),
$$

where the convergence is in the operator norm topology. Let $\Phi=\left(\Phi_{1}, \ldots, \Phi_{p}\right)$, where $\Phi_{1}, \ldots, \Phi_{p}$ are free holomorphic functions on $\mathbf{D}_{f, \text { rad }}^{m}(\mathcal{H})$ with coefficients in $B(\mathcal{Y})$, and the property that range $\Phi \subseteq$ $\mathbf{D}_{g, \text { rad }}^{l}(\mathcal{H} \otimes \mathcal{Y})$. Hence, we deduce that

$$
(F \circ \Phi)\left(X_{1}, \ldots, X_{n}\right)=\sum_{k=0}^{\infty} \sum_{\beta \in \mathbb{F}_{p}^{+},|\beta|=k} \Phi_{\beta}\left(X_{1}, \ldots, X_{n}\right) \otimes A_{(\beta)},
$$

where $\Phi_{\beta}:=\Phi_{i_{1}} \cdots \Phi_{i_{k}}$ if $\beta=g_{i_{1}} \cdots g_{i_{k}} \in \mathbb{F}_{p}^{+}$, and the convergence is in the operator norm topology for any $\left(X_{1}, \ldots, X_{n}\right) \in \mathbf{D}_{f, \text { rad }}^{m}(\mathcal{H})$. According to Lemma 3.1, we have $\Phi\left(r W_{1}, \ldots, r W_{n}\right) \in \mathbf{D}_{f, \text { rad }}^{m}\left(F^{2}\left(H_{n}\right) \otimes \mathcal{Y}\right)$ for $r \in[0,1)$. Taking into account that $F$ is free holomorphic function on $\mathbf{D}_{f, \text { rad }}^{m}(\mathcal{K})$, we deduce that, for each $r \in[0,1)$,

$$
Q_{r}:=\sum_{k=0}^{\infty} \sum_{\beta \in \mathbb{F}_{p}^{+},|\beta|=k} \Phi_{\beta}\left(r W_{1}, \ldots, r W_{n}\right) \otimes A_{(\beta)}
$$

is convergent in the operator norm topology. Since $\Phi_{i}\left(r W_{1}, \ldots, r W_{n}\right)$ is in the tensor algebra $\mathcal{A}_{n}\left(\mathbf{D}_{f}^{m}\right) \bar{\otimes}_{m i n} B(\mathcal{Y})$ for each $i=1, \ldots, p$, the operator $Q_{r}$ is in the operator algebra

$$
\mathcal{A}_{n}\left(\mathbf{D}_{f}^{m}\right) \bar{\otimes}_{\min } B(\mathcal{Y} \otimes \mathcal{E}) \subset F_{n}^{\infty}\left(\mathbf{D}_{f}^{m}\right) \bar{\otimes} B(\mathcal{Y} \otimes \mathcal{E}) .
$$

Therefore, $M_{r}$ has a Fourier representation $\sum_{k=0}^{\infty} \sum_{\alpha \in \mathbb{F}_{n}^{+},|\alpha|=k} r^{|\alpha|} W_{\alpha} \otimes B_{(\alpha)}(r)$, where $B_{(\alpha)}(r) \in B(\mathcal{Y} \otimes$ $\mathcal{E}), \alpha \in \mathbb{F}_{n}^{+}$, and

$$
Q_{r}=\text { SOT }-\lim _{\gamma \rightarrow 1} \sum_{k=0}^{\infty} \sum_{\alpha \in \mathbb{F}_{n}^{+},|\alpha|=k} r^{|\alpha|} \gamma^{|\alpha|} W_{\alpha} \otimes B_{(\alpha)}(r),
$$

where the series $\sum_{k=0}^{\infty} \sum_{\alpha \in \mathbb{F}_{n}^{+},|\alpha|=k} r^{|\alpha|} \gamma^{|\alpha|} W_{\alpha} \otimes B_{(\alpha)}(r)$ converges in the operator norm topology.

Now, we show that the coefficients $B_{(\alpha)}(r), \alpha \in \mathbb{F}_{n}^{+}$, don't depend on $r \in[0,1)$. Taking into account relations (3.2) and (3.3), we have

$$
\begin{aligned}
\left\langle B_{\alpha}(r)(y \otimes x), y^{\prime} \otimes z\right\rangle & =\left\langle\left(W_{\alpha}^{*} \otimes I_{\mathcal{Y} \otimes \mathcal{E})} \frac{b_{\alpha}^{(m)}}{r^{|\alpha|}} Q_{r}(1 \otimes y \otimes x), 1 \otimes y^{\prime} \otimes z\right\rangle\right. \\
& =\lim _{q \rightarrow \infty} \sum_{k=0}^{q} \sum_{\beta \in \mathbb{F}_{p}^{+},|\beta|=k}\left\langle A_{(\beta)} x, z\right\rangle\left\langle\frac{b_{\alpha}^{(m)}}{r^{|\alpha|}}\left(W_{\alpha}^{*} \otimes I_{\mathcal{Y}}\right) \Phi_{\beta}\left(r W_{1}, \ldots, r W_{n}\right)(1 \otimes y), 1 \otimes y^{\prime}\right\rangle
\end{aligned}
$$

for any $x, z \in \mathcal{E}, y, y^{\prime} \in \mathcal{Y}$, and $\alpha \in \mathbb{F}_{n}^{+}$. On the other hand, for each $\beta \in \mathbb{F}_{p}^{+}$, $\Phi_{\beta}$ is a free holomorphic function on $\mathbf{D}_{f, \text { rad }}^{m}(\mathcal{H})$ with coefficients in $B(\mathcal{Y})$ and has a representation $\Phi_{\beta}\left(X_{1}, \ldots, X_{n}\right)=$ $\sum_{k=0}^{\infty} \sum_{\alpha \in \mathbb{F}_{n}^{+},|\alpha|=k} X_{\alpha} \otimes D_{(\alpha)}$ for $\left(X_{1}, \ldots, X_{n}\right) \in \mathbf{D}_{f, \text { rad }}^{m}(\mathcal{H})$, where $D_{(\alpha)} \in B(\mathcal{Y})$. This implies

$$
\left\langle\frac{1}{r^{|\alpha|}}\left(W_{\alpha}^{*} \otimes I_{\mathcal{Y}}\right) \Phi_{\beta}\left(r W_{1}, \ldots, r W_{n}\right)(1 \otimes y), 1 \otimes y^{\prime}\right\rangle=\frac{1}{b_{\alpha}^{(m)}}\left\langle D_{(\alpha)} y, y^{\prime}\right\rangle
$$

for any $r \in[0,1)$, and $\alpha, \beta \in \mathbb{F}_{n}^{+}$. Now, it is clear that $B_{(\alpha)}:=B_{(\alpha)}(r)$ does not depend on $r \in[0,1)$. Now relation $(3.3)$ becomes

$$
Q_{r}=\text { SOT- } \lim _{\gamma \rightarrow 1} \sum_{k=0}^{\infty} \sum_{\alpha \in \mathbb{F}_{n}^{+},|\alpha|=k} r^{|\alpha|} \gamma^{|\alpha|} W_{\alpha} \otimes B_{(\alpha)}
$$


where the series $\sum_{k=0}^{\infty} \sum_{\alpha \in \mathbb{F}_{n}^{+},|\alpha|=k} r^{|\alpha|} \gamma^{|\alpha|} W_{\alpha} \otimes B_{(\alpha)}$ converges in the operator norm topology, for any $r, \gamma \in[0,1)$. According to Theorem 1.2 .

$$
G\left(X_{1}, \ldots, X_{n}\right):=\sum_{k=0}^{\infty} \sum_{\alpha \in \mathbb{F}_{n},|\alpha|=k} X_{\alpha} \otimes B_{(\alpha)}, \quad\left(X_{1}, \ldots, X_{n}\right) \in \mathbf{D}_{f, \text { rad }}^{m}(\mathcal{H})
$$

is a free holomorphic function on $\mathbf{D}_{f, \text { rad }}^{m}(\mathcal{H})$. Using the continuity of $G$ in the norm operator topology and relations (3.2) and (3.4), we deduce that

$$
Q_{r}:=\sum_{k=0}^{\infty} \sum_{\beta \in \mathbb{F}_{p}^{+},|\beta|=k} \Phi_{\beta}\left(r W_{1}, \ldots, r W_{n}\right) \otimes A_{(\beta)}=\sum_{k=0}^{\infty} \sum_{\alpha \in \mathbb{F}_{n}^{+},|\alpha|=k} r^{|\alpha|} W_{\alpha} \otimes B_{(\alpha)}
$$

for any $r \in[0,1)$.

Let $X:=\left(X_{1}, \ldots, X_{n}\right) \in \mathbf{D}_{f, \text { rad }}^{m}(\mathcal{H})$. Then there exists $\gamma \in(0,1)$ such that $\frac{1}{\gamma} X \in \mathbf{D}_{f}^{m}(\mathcal{H})$. Applying the noncommutative Berezin transform at $\left(\frac{1}{\gamma} X_{1}, \ldots, \frac{1}{\gamma} X_{n}\right) \in \mathbf{D}_{f}^{m}(\mathcal{H})$ to relation (3.5), when $r:=\gamma$, we deduce that

$$
(F \circ \Phi)\left(X_{1}, \ldots, X_{n}\right)=\sum_{k=0}^{\infty} \sum_{\beta \in \mathbb{F}_{p}^{+},|\beta|=k} \Phi_{\beta}\left(X_{1}, \ldots, X_{n}\right) \otimes A_{(\beta)}=\sum_{k=0}^{\infty} \sum_{\alpha \in \mathbb{F}_{n}^{+},|\alpha|=k} X_{\alpha} \otimes B_{(\alpha)}
$$

for any $\left(X_{1}, \ldots, X_{n}\right) \in \mathbf{D}_{f, \text { rad }}^{m}(\mathcal{H})$. This completes the proof.

Now, we present results concerning the composition of bounded free holomorphic functions, with operator-valued coefficients, on noncommutative domains $\mathbf{D}_{\text {frad }}^{m}, m \geq 1$.

Theorem 3.3. Let $f$ and $g$ be positive regular free holomorphic functions with $n$ and $p$ indeterminates, respectively, and let $m, l \geq 1$. Let $F: \mathbf{D}_{g, \text { rad }}^{l}(\mathcal{K}) \rightarrow B(\mathcal{K}) \bar{\otimes}_{\min } B(\mathcal{E})$ and $\Phi: \mathbf{D}_{f, \text { rad }}^{m}(\mathcal{H}) \rightarrow$ $\left[B(\mathcal{H}) \bar{\otimes}_{\min } B(\mathcal{Y})\right]^{p}$ be bounded free holomorphic functions. If $F$ and $\Phi$ have continuous extensions to the noncommutative domains $\mathbf{D}_{g}^{l}(\mathcal{K})$ and $\mathbf{D}_{f}^{m}(\mathcal{H})$, respectively, and range $\Phi \subseteq \mathbf{D}_{g}^{l}(\mathcal{H} \otimes \mathcal{Y})$, then $F \circ \Phi$ is a bounded free holomorphic function on $\mathbf{D}_{f, \text { rad }}^{m}(\mathcal{H})$ which has continuous extension to $\mathbf{D}_{f}^{m}(\mathcal{H})$.

Moreover, we have

(a) $\|F \circ \Phi\|_{\infty} \leq\|F\|_{\infty}$;

(b) $(F \circ \Phi)(X)=\left(\mathcal{B}_{X} \otimes I_{\mathcal{Y} \otimes \mathcal{E}}\right)\left\{\left(\mathcal{B}_{\widetilde{\Phi}} \otimes I_{\mathcal{E}}\right)[\widetilde{F}]\right\}, X \in \mathbf{D}_{f}^{m}(\mathcal{H})$, where $\mathcal{B}_{X}, \mathcal{B}_{\widetilde{\Phi}}$ are the noncommutative Berezin transforms at $X$ and $\widetilde{\Phi}$, respectively;

(c) the model boundary function of the composition $F \circ \Phi$ satisfies the equation

$$
\widetilde{F \circ \Phi}=\lim _{r \rightarrow 1} F\left(r \widetilde{\Phi}_{1}, \ldots, r \widetilde{\Phi}_{p}\right)=\left(\mathcal{B}_{\widetilde{\Phi}} \otimes I_{\mathcal{E}}\right)[\widetilde{F}],
$$

where the convergence is in the operator norm topology and $\mathcal{B}_{\widetilde{\Phi}}$ is the noncommutative Berezin transform at $\widetilde{\Phi}$.

Proof. Let $F$ have the representation

$$
F\left(Y_{1}, \ldots, Y_{p}\right):=\sum_{k=0}^{\infty} \sum_{\beta \in \mathbb{F}_{p}^{+},|\beta|=k} Y_{\beta} \otimes A_{(\beta)}, \quad\left(Y_{1}, \ldots, Y_{p}\right) \in \mathbf{D}_{g, \text { rad }}^{l}(\mathcal{K}),
$$

where the series converges in the operator norm topology. Since $F$ and $\Phi$ have continuous extensions to the noncommutative domains $\mathbf{D}_{g}^{l}(\mathcal{K})$ and $\mathbf{D}_{f}^{m}(\mathcal{H})$, respectively, the operator-valued version of Theorem 2.2 implies that $\widetilde{F} \in \mathcal{A}_{p}\left(\mathbf{D}_{g}^{l}\right) \bar{\otimes}_{\text {min }} B(\mathcal{E})$ and $\widetilde{\Phi}:=\left(\widetilde{\Phi}_{1}, \ldots, \widetilde{\Phi}_{p}\right)$ is such that $\widetilde{\Phi}_{j} \in \mathcal{A}_{n}\left(\mathbf{D}_{f}^{m}\right) \bar{\otimes}_{\text {min }} B(\mathcal{Y})$, $j=1, \ldots, p$. Due to the properties of the noncommutative Berezin transform $\mathcal{B}_{\widetilde{\Phi}}$ and the fact that $\left(\widetilde{\Phi}_{1}, \ldots, \widetilde{\Phi}_{p}\right)$ is in $\mathbf{D}_{g}^{l}\left(F^{2}\left(H_{n}\right) \otimes \mathcal{Y}\right)$,

$$
\left.\lim _{r \rightarrow 1} F\left(r \widetilde{\Phi}_{1}, \ldots, r \widetilde{\Phi}_{p}\right)\right)=\lim _{r \rightarrow 1} \sum_{k=0}^{\infty} \sum_{\beta \in \mathbb{F}_{p}^{+},|\beta|=k} r \mid r^{|\beta|} \widetilde{\Phi}_{\beta} \otimes A_{(\beta)}=\left(\mathcal{B}_{\widetilde{\Phi}} \otimes I_{\mathcal{E}}\right)[\widetilde{F}]
$$


where the convergence is in the operator norm topology. Hence and due to the fact that $\mathcal{A}_{n}\left(\mathbf{D}_{f}^{m}\right) \bar{\otimes}_{\min } B(\mathcal{Y} \otimes$ $\mathcal{E})$ is closed in the operator norm, we deduce that $\lim _{r \rightarrow 1} F\left(r \widetilde{\Phi}_{1}, \ldots, r \widetilde{\Phi}_{p}\right)$ is in $\mathcal{A}_{n}\left(\mathbf{D}_{f}^{m}\right) \bar{\otimes}_{\min } B(\mathcal{Y} \otimes \mathcal{E})$. If $X \in \mathbf{D}_{f}^{m}(\mathcal{H})$, then using the continuity of $F, \Phi$, and the continuity of the noncommutative Berezin transform $\mathcal{B}_{X}$ in the operator norm topology, we obtain

$$
\begin{aligned}
(F \circ \Phi)(X) & =\lim _{r \rightarrow 1} F\left(r \Phi_{1}(X), \ldots, r \Phi_{p}(X)\right) \\
& =\lim _{r \rightarrow 1} \sum_{k=0}^{\infty} \sum_{\beta \in \mathbb{F}_{p}^{+},|\beta|=k} r^{|\beta|} \Phi_{\beta}(X) \otimes A_{(\beta)} \\
& =\lim _{r \rightarrow 1} \sum_{k=0}^{\infty} \sum_{\beta \in \mathbb{F}_{p}^{+},|\beta|=k} r^{|\beta|}\left(\mathcal{B}_{X} \otimes I_{\mathcal{Y}}\right)\left[\widetilde{\Phi}_{\beta}\right] \otimes A_{(\beta)} \\
& =\lim _{r \rightarrow 1}\left(\mathcal{B}_{X} \otimes I_{\mathcal{Y} \otimes \mathcal{E}}\right)\left(\sum_{k=0}^{\infty} \sum_{\beta \in \mathbb{F}_{p}^{+},|\beta|=k} r^{|\beta|} \widetilde{\Phi}_{\beta} \otimes A_{(\beta)}\right) \\
& =\left(\mathcal{B}_{X} \otimes I_{\mathcal{Y} \otimes \mathcal{E}}\right) \lim _{r \rightarrow 1}\left(\sum_{k=0}^{\infty} \sum_{\beta \in \mathbb{F}_{p}^{+},|\beta|=k} r^{|\beta|} \widetilde{\Phi}_{\beta} \otimes A_{(\beta)}\right) \\
& =\left(\mathcal{B}_{X} \otimes I_{\mathcal{Y} \otimes \mathcal{E})}\right)\left\{\left(\mathcal{B}_{\widetilde{\Phi}} \otimes I_{\mathcal{E}}\right)[\widetilde{F}]\right\},
\end{aligned}
$$

which proves part (b). Since $\left(\mathcal{B}_{\widetilde{\Phi}} \otimes I_{\mathcal{E}}\right)[\widetilde{F}] \in \mathcal{A}_{n}\left(\mathbf{D}_{f}^{m}\right) \bar{\otimes}_{\min } B(\mathcal{Y} \otimes \mathcal{E})$, the operator-valued version of Theorem 2.2 shows that $F \circ \Phi$ is a free holomorphic function on $\mathbf{D}_{f, \text { rad }}^{m}(\mathcal{H})$ and continuous on $\mathbf{D}_{f}^{m}(\mathcal{H})$ and its model boundary function satisfies the equation

$$
\widetilde{F \circ \Phi}=\lim _{r \rightarrow 1} F\left(r \widetilde{\Phi}_{1}, \ldots, r \widetilde{\Phi}_{p}\right)=\left(\mathcal{B}_{\widetilde{\Phi}} \otimes I_{\mathcal{E}}\right)[\widetilde{F}]
$$

which proves part (c). Part (a) is now obvious. The proof is complete.

We recall that an $n$-tuple $T:=\left(T_{1}, \ldots, T_{n}\right) \in \mathbf{D}_{f}^{m}(\mathcal{H})$ is pure if SOT- $\lim _{k \rightarrow \infty} \Phi_{f, T}^{k}(I)=0$, where $\Phi_{f, T}: B(\mathcal{H}) \rightarrow B(\mathcal{H})$ is the positive linear map defined by $\Phi_{f, T}(X)=\sum_{k=1}^{\infty} \sum_{|\alpha|=k} a_{\alpha} T_{\alpha} X T_{\alpha}^{*}$, where the convergence is in the weak operator topology. We define

$$
\mathbf{D}_{f, \text { pure }}^{m}(\mathcal{H}):=\left\{\left(X_{1}, \ldots, X_{n}\right) \in \mathbf{D}_{f}^{m}(\mathcal{H}):\left(X_{1}, \ldots, X_{n}\right) \text { is pure }\right\} .
$$

Note that $\mathbf{D}_{f, \text { rad }}^{m}(\mathcal{H}) \subset \mathbf{D}_{f, \text { pure }}^{m}(\mathcal{H}) \subset \mathbf{D}_{f}^{m}(\mathcal{H})$.

Lemma 3.4. Let $f$ and $g$ be positive regular free holomorphic functions with $n$ and $p$ indeterminates, respectively, and let $m, l \geq 1$. Let $\psi_{i} \in H^{\infty}\left(\mathbf{D}_{f}^{m}\right), i=1, \ldots, p$, be such that $\widetilde{\psi}:=\left(\widetilde{\psi}_{1}, \ldots, \widetilde{\psi}_{p}\right)$ is in $\mathbf{D}_{g, \text { pure }}^{l}\left(F^{2}\left(H_{n}\right)\right)$, where $\widetilde{\psi}_{i} \in F_{n}^{\infty}\left(\mathbf{D}_{f}^{m}\right)$ is the model boundary function. Then

$$
\psi(X):=\left(\psi_{1}(X), \ldots, \psi_{p}(X)\right) \in \mathbf{D}_{g, \text { pure }}^{l}(\mathcal{H})
$$

for any $X:=\left(X_{1}, \ldots, X_{n}\right) \in \mathbf{D}_{f, \text { pure }}^{m}(\mathcal{H})$.

Proof. Taking into account that $\widetilde{\psi}:=\left(\widetilde{\psi}_{1}, \ldots, \widetilde{\psi}_{p}\right) \in \mathbf{D}_{g, \text { pure }}^{l}\left(F^{2}\left(H_{n}\right)\right)$ and $X:=\left(X_{1}, \ldots, X_{n}\right) \in \mathbf{D}_{f, \text { pure }}^{m}(\mathcal{H})$, and using the properties of the extended noncommutative Berezin transform, we deduce that

$$
\psi_{\alpha}(X) \psi_{\alpha}(X)^{*}=K_{f, X}^{(m)^{*}}\left(\widetilde{\psi}_{\alpha} \widetilde{\psi}_{\beta}^{*} \otimes I_{\mathcal{H}}\right) K_{f, X}^{(m)}, \quad \alpha \in \mathbb{F}_{n}^{+} .
$$

Hence, we obtain

$$
\Phi_{g, \psi(X)}^{k}(I)=K_{f, X}^{(m)^{*}}\left(\Phi_{g, \widetilde{\psi}}^{k}(I) \otimes I_{\mathcal{H}}\right) K_{f, X}^{(m)}, \quad k \in \mathbb{N} .
$$

Since $\Phi_{g, \widetilde{\psi}}^{k}(I) \leq I$ for any $k \in \mathbb{N}$ and SOT- $\lim _{k \rightarrow \infty} \Phi_{g, \widetilde{\psi}}^{k}(I)=0$, we deduce that

$$
\text { SOT- } \lim _{k \rightarrow \infty} \Phi_{g, \psi(X)}^{k}(I)=0,
$$

which shows that $\psi(X)$ is pure. 
Theorem 3.5. Let $f$ and $g$ be positive regular free holomorphic functions with $n$ and $p$ indeterminates, respectively, and let $m, l \geq 1$. Let $F: \mathbf{D}_{g, \text { rad }}^{l}(\mathcal{K}) \rightarrow B(\mathcal{K}) \bar{\otimes}_{\min } B(\mathcal{E})$ and $\Phi: \mathbf{D}_{f, \text { rad }}^{m}(\mathcal{H}) \rightarrow B(\mathcal{H})^{p}$ be bounded free holomorphic functions. If the model boundary function $\widetilde{\Phi}$ is a pure $n$-tuple in $\mathbf{D}_{g}^{l}\left(F^{2}\left(H_{n}\right)\right)$, then the map

$$
(F \circ \Phi)(X):=\text { SOT- } \lim _{r \rightarrow 1} F\left(r \Phi_{1}(X), \ldots, r \Phi_{n}(X)\right), \quad X \in \mathbf{D}_{f, \text { pure }}^{m}(\mathcal{H}),
$$

is a bounded free holomorphic function on $\mathbf{D}_{f, \text { rad }}^{m}(\mathcal{H})$. Moreover, we have

(a) $\|F \circ \Phi\|_{\infty} \leq\|F\|_{\infty}$;

(b) $(F \circ \Phi)(X)=\left(\mathcal{B}_{X} \otimes I_{\mathcal{E}}\right)\left\{\left(\mathcal{B}_{\widetilde{\Phi}} \otimes I_{\mathcal{E}}\right)[\widetilde{F}]\right\}, X \in \mathbf{D}_{f, \text { pure }}^{m}(\mathcal{H})$, where $\mathcal{B}_{X}, \mathcal{B}_{\widetilde{\Phi}}$ are the noncommutative Berezin transforms at $X$ and $\widetilde{\Phi}$, respectively;

(c) the model boundary function of the composition $F \circ \Phi$ satisfies the equation

$$
\widetilde{F \circ \Phi}=\mathrm{SOT}-\lim _{r \rightarrow 1} F\left(r \widetilde{\Phi}_{1}, \ldots, r \widetilde{\Phi}_{p}\right)=\left(\mathcal{B}_{\widetilde{\Phi}} \otimes I_{\mathcal{E}}\right)[\widetilde{F}]
$$

where $\mathcal{B}_{\widetilde{\Phi}}$ is the noncommutative Berezin transform at $\widetilde{\Phi}$.

Proof. Let $F$ have the representation

$$
F\left(Y_{1}, \ldots, Y_{p}\right):=\sum_{k=0}^{\infty} \sum_{\beta \in \mathbb{F}_{p}^{+},|\beta|=k} Y_{\beta} \otimes A_{(\beta)}, \quad\left(Y_{1}, \ldots, Y_{p}\right) \in \mathbf{D}_{g, \mathrm{rad}}^{l}(\mathcal{K}),
$$

where the series converges in the operator norm topology. Since $F$ and $\Phi$ are bounded free holomorphic functions on the noncommutative domains $\mathbf{D}_{g \text {,rad }}^{l}(\mathcal{K})$ and $\mathbf{D}_{f, \text { rad }}^{m}(\mathcal{H})$, respectively, the operator-valued version of Theorem 2.1 implies that $\widetilde{F} \in F_{n}^{\infty}\left(\mathbf{D}_{g}^{l}\right) \bar{\otimes} B(\mathcal{E})$ and $\widetilde{\Phi}:=\left(\widetilde{\Phi}_{1}, \ldots, \widetilde{\Phi}_{p}\right)$ is such that $\widetilde{\Phi}_{j} \in$ $F_{n}^{\infty}\left(\mathbf{D}_{f}^{m}\right), j=1, \ldots, p$. Due to the properties of the noncommutative Berezin transform $\mathcal{B}_{\widetilde{\Phi}}$ and the fact that $\left(\widetilde{\Phi}_{1}, \ldots, \widetilde{\Phi}_{p}\right)$ is a pure $n$-tuple in $\mathbf{D}_{g}^{l}\left(F^{2}\left(H_{n}\right)\right)$, we have

$$
\text { SOT- } \left.\lim _{r \rightarrow 1} F\left(r \widetilde{\Phi}_{1}, \ldots, r \widetilde{\Phi}_{p}\right)\right)=\text { SOT- } \lim _{r \rightarrow 1} \sum_{k=0}^{\infty} \sum_{\beta \in \mathbb{F}_{p}^{+},|\beta|=k} r^{|\beta|} \widetilde{\Phi}_{\beta} \otimes A_{(\beta)}=\left(\mathcal{B}_{\widetilde{\Phi}} \otimes I_{\mathcal{E}}\right)[\widetilde{F}] .
$$

Hence and due to the fact that $F_{n}^{\infty}\left(\mathbf{D}_{f}^{m}\right) \bar{\otimes} B(\mathcal{E})$ is closed in the strong operator topology, we deduce that SOT- $\lim _{r \rightarrow 1} F\left(r \widetilde{\Phi}_{1}, \ldots, r \widetilde{\Phi}_{p}\right)$ is in $F_{n}^{\infty}\left(\mathbf{D}_{f}^{m}\right) \bar{\otimes} B(\mathcal{E})$. If $X \in \mathbf{D}_{f, \text { pure }}^{m}(\mathcal{H})$, then Lemma 3.4 implies that $\left(\Phi_{1}(X), \ldots, \Phi_{p}(X)\right)$ is a pure $n$-tuple of operators in $\mathbf{D}_{g}^{l}(\mathcal{H})$. Consequently, as in the proof of Theorem 3.3. using the continuity of the noncommutative Berezin transform $\mathcal{B}_{X}$ in the strong operator topology (see (2.1)), and relation (3.6), we obtain

$$
\begin{aligned}
(F \circ \Phi)(X) & =\text { SOT- } \lim _{r \rightarrow 1} F\left(r \Phi_{1}(X), \ldots, r \Phi_{p}(X)\right) \\
& =\text { SOT- } \lim _{r \rightarrow 1} \sum_{k=0}^{\infty} \sum_{\beta \in \mathbb{F}_{p}^{+},|\beta|=k} r^{|\beta|} \Phi_{\beta}(X) \otimes A_{(\beta)} \\
& =\left(\mathcal{B}_{X} \otimes I_{\mathcal{E}}\right)\left\{\left(\mathcal{B}_{\widetilde{\Phi}} \otimes I_{\mathcal{E}}\right)[\widetilde{F}]\right\},
\end{aligned}
$$

which proves part (b). Since $\left(\mathcal{B}_{\widetilde{\Phi}} \otimes I_{\mathcal{E}}\right)[\widetilde{F}] \in F_{n}^{\infty}\left(\mathbf{D}_{f}^{m}\right) \bar{\otimes}_{\min } B(\mathcal{E})$, the operator-valued version of Theorem 2.2 shows that $F \circ \Phi$ is a free holomorphic function on $\mathbf{D}_{f, \text { rad }}^{m}(\mathcal{H})$ and its model boundary function satisfies the equation

$$
\widetilde{F \circ \Phi}=\mathrm{SOT}-\lim _{r \rightarrow 1} F\left(r \widetilde{\Phi}_{1}, \ldots, r \widetilde{\Phi}_{p}\right)=\left(\mathbf{B}_{\widetilde{\Phi}} \otimes I_{\mathcal{E}}\right)[\widetilde{F}],
$$

which proves part (c). Part (a) is now obvious. The proof is complete. 


\section{Free Biholomorphic functions and noncommutative Cartan type Results}

In this section we obtain noncommutative Cartan type results for formal power series in several noncommuting indeterminates, which leave invariant the nilpotent parts of the corresponding domains. These results are used to characterize the set of all free biholomorphic functions $F: \mathbf{D}_{f}^{m}(\mathcal{H}) \rightarrow \mathbf{D}_{g}^{l}(\mathcal{H})$ with $F(0)=0$. As a consequence, we determine the free holomorphic automorphisms of the noncommutative domain $\mathbf{D}_{f, \text { rad }}^{m}$ which fix the origin. Several other consequences concerning the biholomorphic classification of noncommutative domains are obtained.

An $n$-tuple of operators $\left(N_{1}, \ldots, N_{n}\right) \in B(\mathcal{H})^{n}$ is called nilpotent if there is $p \in \mathbb{N}:=\{1,2, \ldots\}$ such that $N_{\alpha}=0$ for any $\alpha \in \mathbb{F}_{n}^{+}$with $|\alpha|=p$. Define the nilpotent part of the noncommutative domain $\mathbf{D}_{f}^{m}(\mathcal{H})$ by setting

$$
\mathbf{D}_{f, \text { nil }}^{m}(\mathcal{H}):=\left\{\left(N_{1}, \ldots, N_{n}\right) \in \mathbf{D}_{f}^{m}(\mathcal{H}):\left(N_{1}, \ldots, N_{n}\right) \text { is nilpotent }\right\} .
$$

For simplicity, throughout this paper, $\left[X_{1}, \ldots, X_{n}\right]$ denotes either the $n$-tuple $\left(X_{1}, \ldots, X_{n}\right) \in B(\mathcal{H})^{n}$ or the operator row matrix $\left[X_{1} \cdots X_{n}\right]$ acting from $\mathcal{H}^{(n)}$, the direct sum of $n$ copies of a Hilbert space $\mathcal{H}$, to $\mathcal{H}$.

Theorem 4.1. Let $f$ be a positive regular free holomorphic function with $n$ indeterminates and let $m \geq 1$. Let $H_{1}, \ldots, H_{n}$ be formal power series in $n$ noncommuting indeterminates $Z_{1}, \ldots, Z_{n}$ of the form

$$
H_{i}\left(Z_{1}, \ldots, Z_{n}\right):=\sum_{k=2} \sum_{|\alpha|=k} a_{\alpha}^{(i)} Z_{\alpha}, \quad a_{\alpha}^{(i)} \in \mathbb{C}, i=1, \ldots, n .
$$

If

$$
F\left(Z_{1}, \ldots, Z_{n}\right):=\left(Z_{1}+H_{1}\left(Z_{1}, \ldots, Z_{n}\right), \ldots, Z_{n}+H_{n}\left(Z_{1}, \ldots, Z_{n}\right)\right)
$$

has the property that

for any Hilbert space $\mathcal{H}$, then

$$
F\left(\mathbf{D}_{f, \text { nil }}^{m}(\mathcal{H})\right) \subseteq \mathbf{D}_{f, \text { nil }}^{m}(\mathcal{H})
$$

$$
F\left(Z_{1}, \ldots, Z_{n}\right)=\left(Z_{1}, \ldots, Z_{n}\right)
$$

Proof. Assume that there exists $\alpha \in \mathbb{F}_{n}^{+},|\alpha| \geq 2$, and $i \in\{1, \ldots, n\}$ such that $a_{\alpha}^{(i)} \neq 0$. Let $p \geq 2$ be the smallest natural number such that there exists $\alpha_{0} \in \mathbb{F}_{n}^{+},\left|\alpha_{0}\right|=p$, and $i_{0} \in\{1, \ldots, n\}$ such that $a_{\alpha_{0}}^{\left(i_{0}\right)} \neq 0$. Then $F$ has the form $F=\left(F_{1}, \ldots, F_{n}\right)$, where, for each $i=1, \ldots, n$,

$$
F_{i}\left(Z_{1}, \ldots, Z_{n}\right)=Z_{i}+H_{i}\left(Z_{1}, \ldots, Z_{n}\right) \quad \text { and } \quad H_{i}\left(Z_{1}, \ldots, Z_{n}\right)=\sum_{k=p}^{\infty} G_{k}^{(i)}\left(Z_{1}, \ldots, Z_{n}\right)
$$

where $G_{k}^{(i)}\left(Z_{1}, \ldots, Z_{n}\right):=\sum_{|\alpha|=k} a_{\alpha}^{(i)} Z_{\alpha}$ for each $k \geq p$ and $i \in\{1, \ldots, n\}$. Note that, for each $i \in$ $\{1, \ldots, n\}, G_{p}^{(i)} \circ F$ is a power series and

$$
\left(G_{p}^{(i)} \circ F\right)\left(Z_{1}, \ldots, Z_{n}\right)=\sum_{|\alpha|=p} a_{\alpha}^{(i)} F_{\alpha}\left(Z_{1}, \ldots, Z_{n}\right)=G_{p}^{(i)}\left(Z_{1}, \ldots, Z_{n}\right)+K_{p+1}^{(i)}\left(Z_{1}, \ldots, Z_{n}\right),
$$

where $K_{p+1}^{(i)}$ is a power series containing only monomials of degree $\geq p+1$ in its representation. Therefore, $F \circ F$ is a power series and

$$
\begin{gathered}
(F \circ F)\left(Z_{1}, \ldots, Z_{n}\right)=\left(Z_{1}, \ldots, Z_{n}\right)+\left(2 G_{p}^{(1)}\left(Z_{1}, \ldots, Z_{n}\right), \ldots, 2 G_{p}^{(n)}\left(Z_{1}, \ldots, Z_{n}\right)\right) \\
+\left(K_{p+1}^{(1)}\left(Z_{1}, \ldots, Z_{n}\right), \ldots, K_{p+1}^{(n)}\left(Z_{1}, \ldots, Z_{n}\right)\right) .
\end{gathered}
$$

Iterating this process and setting $F^{N}:=\underbrace{(F \circ \cdots \circ F)}_{N \text { times }}, N \in \mathbb{N}$, we deduce that

$$
\begin{gathered}
F^{N}\left(Z_{1}, \ldots, Z_{n}\right)=\left(Z_{1}, \ldots, Z_{n}\right)+\left(N G_{p}^{(1)}\left(Z_{1}, \ldots, Z_{n}\right), \ldots, N G_{p}^{(n)}\left(Z_{1}, \ldots, Z_{n}\right)\right) \\
+\left(E_{p+1}^{(1)}\left(Z_{1}, \ldots, Z_{n}\right), \ldots, E_{p+1}^{(n)}\left(Z_{1}, \ldots, Z_{n}\right)\right),
\end{gathered}
$$


where, for each $i=1, \ldots, n, E_{p+1}^{(i)}$ is a power series containing only monomials of degree $\geq p+1$ in its representation. Let $\mathcal{M} \subset F^{2}\left(\mathcal{H}_{n}\right)$ be the linear span generated by $e_{\alpha}$, where $\alpha \in \mathbb{F}_{n}^{+}$with $|\alpha| \leq p$. Note that $\mathcal{M}$ is an invariant subspace under $W_{1}^{*}, \ldots, W_{n}^{*}$. Using the definition of the weighted shifts $W_{1}, \ldots, W_{n}$ associated with the noncommutative domain $\mathbf{D}_{f}^{m}$, we deduce that

$$
\left[F^{N}\left(\left.P_{\mathcal{M}} W_{1}\right|_{\mathcal{M}}, \ldots,\left.P_{\mathcal{M}} W_{n}\right|_{\mathcal{M}}\right)\right]^{*} e_{\alpha}=\left[\begin{array}{c}
W_{1}^{*} \\
\vdots \\
W_{n}^{*}
\end{array}\right] e_{\alpha}+N\left[\begin{array}{c}
{\left[G_{p}^{(1)}\left(\left.P_{\mathcal{M}} W_{1}\right|_{\mathcal{M}}, \ldots,\left.P_{\mathcal{M}} W_{n}\right|_{\mathcal{M}}\right)\right]^{*}} \\
\vdots \\
{\left[G_{p}^{(n)}\left(\left.P_{\mathcal{M}} W_{1}\right|_{\mathcal{M}}, \ldots,\left.P_{\mathcal{M}} W_{n}\right|_{\mathcal{M}}\right)\right]^{*}}
\end{array}\right] e_{\alpha}
$$

for any $\alpha \in \mathbb{F}_{n}^{+}$with $|\alpha| \geq 2$, and $N=1,2, \ldots$, where $P_{\mathcal{M}}$ is the orthogonal projection from $F^{2}\left(H_{n}\right)$ onto $\mathcal{M}$. We recall that $\left\{e_{\alpha}\right\}_{\alpha \in \mathbb{F}_{n}^{+}}$is the standard orthonormal basis for $F^{2}\left(H_{n}\right)$. Since, for any $\alpha \in \mathbb{F}_{n}^{+}$, $|\alpha|=p$,

$$
W_{\alpha}^{*} e_{\alpha_{0}}= \begin{cases}\frac{1}{\sqrt{b_{\alpha_{0}}^{(m)}}} 1 & \text { if } \alpha=\alpha_{0} \\ 0 & \text { otherwise }\end{cases}
$$

and $G_{p}^{(i)}, i=1, \ldots, n$, are homogeneous noncommutative polynomials of degree $p$ and $\left|\alpha_{0}\right|=p$, we have

$$
\gamma:=\left\|\left[\begin{array}{c}
{\left[G_{p}^{(1)}\left(\left.P_{\mathcal{M}} W_{1}\right|_{\mathcal{M}}, \ldots,\left.P_{\mathcal{M}} W_{n}\right|_{\mathcal{M}}\right)\right]^{*}} \\
\vdots \\
{\left[G_{p}^{(n)}\left(\left.P_{\mathcal{M}} W_{1}\right|_{\mathcal{M}}, \ldots,\left.P_{\mathcal{M}} W_{n}\right|_{\mathcal{M}}\right)\right]^{*}}
\end{array}\right] e_{\alpha_{0}}\right\| \geq \frac{\left|a_{\alpha_{0}}^{\left(i_{0}\right)}\right|}{\sqrt{b_{\alpha_{0}}^{(m)}}}>0
$$

Assume that $\alpha_{0}=g_{j} \gamma_{0}$, where $j=1, \ldots, n$ and $\gamma_{0} \in \mathbb{F}_{n}^{+},\left|\gamma_{0}\right|=p-1$. Then, due to relation (1.4), we have $W_{j}^{*} e_{\alpha_{0}}=\frac{\sqrt{b_{\gamma_{0}}^{(m)}}}{\sqrt{b_{\alpha_{0}}^{(m)}}} e_{\gamma_{0}}$ and $W_{i}^{*} e_{\alpha_{0}}=0$ for $i \neq j$. Hence and using relation (4.1), we deduce that

$$
N \gamma<\frac{\sqrt{b_{\gamma_{0}}^{(m)}}}{\sqrt{b_{\alpha_{0}}^{(m)}}}+\left\|F^{N}\left(\left.P_{\mathcal{M}} W_{1}\right|_{\mathcal{M}}, \ldots,\left.P_{\mathcal{M}} W_{n}\right|_{\mathcal{M}}\right)^{*} e_{\alpha_{0}}\right\| \quad \text { for any } N \in \mathbb{N} .
$$

On the other hand, since $\left(\left.P_{\mathcal{M}} W_{1}\right|_{\mathcal{M}}, \ldots,\left.P_{\mathcal{M}} W_{n}\right|_{\mathcal{M}}\right)$ is a nilpotent $n$-tuple in $\mathbf{D}_{f}^{m}(\mathcal{M})$ and due to the hypothesis, we have $F^{N}\left(\left.P_{\mathcal{M}} W_{1}\right|_{\mathcal{M}}, \ldots,\left.P_{\mathcal{M}} W_{n}\right|_{\mathcal{M}}\right) \subseteq \mathbf{D}_{f, \text { nil }}^{m}(\mathcal{M})$. Applying inequality (1.1) we obtain

$$
\left\|F^{N}\left(\left.P_{\mathcal{M}} W_{1}\right|_{\mathcal{M}}, \ldots,\left.P_{\mathcal{M}} W_{n}\right|_{\mathcal{M}}\right)\right\|<\frac{1}{\min \left\{\sqrt{a_{\alpha}}:|\alpha|=1\right\}}
$$

for any $N \in \mathbb{N}$, where $a_{\alpha}$ are the coefficients of $f=\sum_{|\alpha| \geq 1} a_{\alpha} X_{\alpha}$. We recall that $a_{\alpha}>0$ if $|\alpha|=1$. Hence and using relation (4.3), we deduce that

$$
N \gamma<\frac{\sqrt{b_{\gamma_{0}}^{(m)}}}{\sqrt{b_{\alpha_{0}}^{(m)}}}+\frac{1}{\min \left\{\sqrt{a_{\alpha}}:|\alpha|=1\right\}}
$$

for any $N \in \mathbb{N}$, which, due to (4.2), is a contradiction. This completes the proof.

In what follows, if $L:=\left[a_{i j}\right]_{n \times n}$ is a bounded linear operator on $\mathbb{C}^{n}$, we use the matrix notation

$$
\left[X_{1}, \ldots, X_{n}\right] L:=\left(\sum_{i=1}^{n} a_{i 1} X_{i}, \cdots, \sum_{i=1}^{n} a_{i n} X_{i}\right) .
$$

Theorem 4.2. Let $f$ and $g$ be positive regular free holomorphic functions with $n$ indeterminates and let $m, l \geq 1$. Let $F=\left(F_{1}, \ldots, F_{n}\right)$ and $G=\left(G_{1}, \ldots, G_{n}\right)$ be $n$-tuples of formal power series in $n$ noncommuting indeterminates such that

$$
F(0)=G(0)=0 \quad \text { and } \quad F \circ G=G \circ F=\text { id. }
$$

If $F\left(\mathbf{D}_{f, \text { nil }}^{m}(\mathcal{H})\right)=\mathbf{D}_{g, \text { nil }}^{l}(\mathcal{H})$ for any Hilbert space $\mathcal{H}$, then $F$ has the form

$$
F\left(Z_{1}, \ldots, Z_{n}\right)=\left[Z_{1}, \ldots, Z_{n}\right] U
$$


where $U$ is an invertible bounded linear operator on $\mathbb{C}^{n}$.

Proof. Since $F(0)=0, F$ has the representation $F=\left(F_{1}, \ldots, F_{n}\right)$, where each $F_{j}$ is a power series with scalar coefficients, having the form

$$
F_{j}\left(Z_{1}, \ldots, Z_{n}\right)=\sum_{k=1}^{n} a_{k j} Z_{k}+\Psi_{2}^{(j)}\left(Z_{1}, \ldots, Z_{n}\right),
$$

and $\Psi_{2}^{(j)}$ is a power series of the form $\Psi_{2}^{(j)}\left(Z_{1}, \ldots, Z_{n}\right)=\sum_{p=2}^{\infty} \sum_{|\alpha|=p} a_{\alpha}^{(j)} Z_{\alpha}, a_{\alpha}^{(j)} \in \mathbb{C}$. Similarly, $G=\left(G_{1}, \ldots, G_{n}\right)$, where each $G_{j}$ is a power series, having the form

$$
G_{j}\left(Z_{1}, \ldots, Z_{n}\right)=\sum_{k=1}^{n} b_{k j} Z_{k}+\Gamma_{2}^{(j)}\left(Z_{1}, \ldots, Z_{n}\right),
$$

and $\Gamma_{2}^{(j)}$ is a power series of the form $\Gamma_{2}^{(j)}\left(Z_{1}, \ldots, Z_{n}\right)=\sum_{p=2}^{\infty} \sum_{|\alpha|=p} b_{\alpha}^{(j)} Z_{\alpha}, b_{\alpha}^{(j)} \in \mathbb{C}$. Consider the matrices $U:=\left[a_{i j}\right]_{n \times n}$ and $B:=\left[b_{i j}\right]_{n \times n}$. Using the representations (4.4) and (4.5), we deduce that $(G \circ F)\left(Z_{1}, \ldots, Z_{n}\right)$

$$
\begin{aligned}
= & \left(\sum_{j=1}^{n} b_{j 1} F_{j}+\Gamma_{2}^{(1)}\left(F_{1}, \ldots, F_{n}\right), \ldots, \sum_{j=1}^{n} b_{j n} F_{j}+\Gamma_{2}^{(n)}\left(F_{1}, \ldots, F_{n}\right)\right) \\
= & \left(\sum_{j=1}^{n} b_{j 1}\left(\sum_{k=1}^{n} a_{k j} Z_{k}\right), \ldots, \sum_{j=1}^{n} b_{j n}\left(\sum_{k=1}^{n} a_{k j} Z_{k}\right)\right) \\
& +\left(\sum_{j=1}^{n} b_{j 1} \Psi_{2}^{(j)}\left(Z_{1}, \ldots, Z_{n}\right), \ldots, \sum_{j=1}^{n} b_{j n} \Psi_{2}^{(j)}\left(Z_{1}, \ldots, Z_{n}\right)\right)+\left(\Gamma_{2}^{(1)}\left(F_{1}, \ldots, F_{n}\right), \ldots, \Gamma_{2}^{(n)}\left(F_{1}, \ldots, F_{n}\right)\right) \\
= & {\left[Z_{1}, \ldots, Z_{n}\right] U B+\left(\Lambda_{2}^{(1)}\left(Z_{1}, \ldots, Z_{n}\right), \ldots, \Lambda_{2}^{(n)}\left(Z_{1}, \ldots, Z_{n}\right)\right) }
\end{aligned}
$$

where $\Lambda_{2}^{(j)}, j=1, \ldots, n$, are power series containing only monomials of degree $\geq 2$ in their representations. Since $(G \circ F)\left(Z_{1}, \ldots, Z_{n}\right)=\left(Z_{1}, \ldots, Z_{n}\right)$, we deduce that $U B=I_{n}$ and $\Lambda_{2}^{(j)}\left(Z_{1}, \ldots, Z_{n}\right)=0$ for $j=$ $1, \ldots, n$. Similarly, since $(F \circ G)\left(Z_{1}, \ldots, Z_{n}\right)=\left(Z_{1}, \ldots, Z_{n}\right)$, we can prove that $B U=I_{n}$. Therefore $U$ is an invertible operator on $\mathbb{C}^{n}$. Note that

$$
F_{\theta}\left(Z_{1}, \ldots, Z_{n}\right):=e^{-i \theta} F\left(e^{i \theta} Z_{1}, \ldots, e^{i \theta} Z_{n}\right)
$$

is a power series, for all $\theta \in \mathbb{R}$. Due to relation (4.4), we deduce that

$$
\begin{aligned}
e^{-i \theta} F\left(e^{i \theta} Z_{1}, \ldots, e^{i \theta} Z_{n}\right) & \\
& =\left(\sum_{k=1}^{n} a_{k 1} Z_{k}+e^{-i \theta} \Psi_{2}^{(1)}\left(e^{i \theta} Z_{1}, \ldots, e^{i \theta} Z_{n}\right), \ldots, \sum_{k=1}^{n} a_{k n} Z_{k}+e^{-i \theta} \Psi_{2}^{(n)}\left(e^{i \theta} Z_{1}, \ldots, e^{i \theta} Z_{n}\right)\right)
\end{aligned}
$$

Note that

$$
H\left(Z_{1}, \ldots, Z_{n}\right):=G\left(e^{-i \theta} F\left(e^{i \theta} Z_{1}, \ldots, e^{i \theta} Z_{n}\right)\right)
$$

is a power series with $H(0)=0$. Taking into account the representations of the power series involved in the definition of $H$, calculations as above lead to

$$
H\left(Z_{1}, \ldots, Z_{n}\right)=\left[Z_{1}, \ldots, Z_{n}\right] U B+\left(\Phi_{2}^{(1)}\left(Z_{1}, \ldots, Z_{n}\right), \ldots, \Phi_{2}^{(n)}\left(Z_{1}, \ldots, Z_{n}\right)\right),
$$

where $\Phi_{2}^{(j)}, j=1, \ldots, n$, are power series containing only monomials of degree $\geq 2$ in their representations. Note that if $\left(N_{1}, \ldots, N_{n}\right) \in \mathbf{D}_{f, \text { nil }}^{m}(\mathcal{H})$, then $\left(e^{i \theta} N_{1}, \ldots, e^{i \theta} N_{n}\right) \in \mathbf{D}_{f \text {,nil }}^{m}(\mathcal{H})$ for $\theta \in \mathbb{R}$, and due to relation $F\left(\mathbf{D}_{f, \text { nil }}^{m}(\mathcal{H})\right)=\mathbf{D}_{g, \text { nil }}^{l}(\mathcal{H})$, we deduce that $F\left(e^{i \theta} N_{1}, \ldots, e^{i \theta} N_{n}\right) \in \mathbf{D}_{g, \text { nil }}^{l}(\mathcal{H})$. Consequently, $e^{i \theta} F\left(e^{i \theta} N_{1}, \ldots, e^{i \theta} N_{n}\right) \in \mathbf{D}_{g, \text { nil }}^{l}(\mathcal{H})$ which together with relation $G\left(\mathbf{D}_{g, \text { nil }}^{l}(\mathcal{H})\right)=\mathbf{D}_{f, \text { nil }}^{m}(\mathcal{H})$ show that the formal power series $H\left(Z_{1}, \ldots, Z_{n}\right):=G\left(e^{-i \theta} F\left(e^{i \theta} Z_{1}, \ldots, e^{i \theta} Z_{n}\right)\right)$ has the properties that $H(0)=0$ and $H\left(\mathbf{D}_{f, \text { nil }}^{m}(\mathcal{H})\right) \subseteq \mathbf{D}_{f, \text { nil }}^{m}(\mathcal{H})$. 
Now, since $U B=I_{n}$, we can apply Theorem 4.1 to the power series $H$ to conclude that $H\left(Z_{1}, \ldots, Z_{n}\right)=$ $\left(Z_{1}, \ldots, Z_{n}\right)$. Hence, taking into account that $G \circ F=i d$ and due to relation (4.6), we obtain

$$
e^{i \theta} F\left(Z_{1}, \ldots, Z_{n}\right)=F\left(e^{i \theta} Z_{1}, \ldots, e^{i \theta} Z_{n}\right)
$$

for any $\theta \in \mathbb{R}$. Using the representations given by (4.4), the latter equality implies

$$
a_{\alpha}^{(j)} e^{i \theta|\alpha|}=e^{i \theta} a_{\alpha}^{(j)} \quad \text { for any } \theta \in \mathbb{R},
$$

where $\alpha \in \mathbb{F}_{n}^{+}$with $|\alpha| \geq 2$, and $j=1, \ldots, n$. Hence, $a_{\alpha}^{(j)}=0$ and, consequently,

$$
F\left(Z_{1}, \ldots, Z_{n}\right)=\left(\sum_{k=1}^{n} a_{k 1} Z_{k}, \ldots, \sum_{k=1}^{n} a_{k n} Z_{k}\right)=\left[Z_{1}, \ldots, Z_{n}\right] U .
$$

The proof is complete.

Let $f$ and $g$ be positive regular free holomorphic functions with $n$ and $q$ indeterminates, respectively, and let $m, l \geq 1$. A map $F: \mathbf{D}_{f}^{m}(\mathcal{H}) \rightarrow \mathbf{D}_{g}^{l}(\mathcal{H})$ is called free biholomorphic function if $F$ is a homeomorphism in the operator norm topology and $\left.F\right|_{\mathbf{D}_{f, \text { rad }}^{m}(\mathcal{H})}$ and $\left.F^{-1}\right|_{\mathbf{D}_{g, \text { rad }}^{l}(\mathcal{H})}$ are free holomorphic functions on $\mathbf{D}_{f, \text { rad }}^{m}(\mathcal{H})$ and $\mathbf{D}_{g \text {,rad }}^{l}(\mathcal{H})$, respectively. In this case, the domains $\mathbf{D}_{f}^{m}(\mathcal{H})$ and $\mathbf{D}_{g}^{l}(\mathcal{H})$ are called free biholomorphic equivalent. We denote by $\operatorname{Bih}\left(\mathbf{D}_{f}^{m}, \mathbf{D}_{g}^{l}\right)$ the set of all the free biholomorphic functions $F: \mathbf{D}_{f}^{m}(\mathcal{H}) \rightarrow \mathbf{D}_{g}^{l}(\mathcal{H})$.

As in the particular case $m=l=1$ (see 29, 2]), there is an important connection between the theory of free biholomorphic functions on noncommutative domains and the theory of biholomorphic functions on domains in $\mathbb{C}^{d}\left([12)\right.$. Let $\Omega_{1}, \Omega_{2}$ be domains (open and connected sets) in $\mathbb{C}^{d}$. If there exist free holomorphic maps $\varphi: \Omega_{1} \rightarrow \Omega_{2}$ and $\psi: \Omega_{2} \rightarrow \Omega_{1}$ such that $\varphi \circ \psi=i d_{\Omega_{2}}$ and $\psi \circ \varphi=i d_{\Omega_{1}}$, then $\Omega_{1}$ and $\Omega_{2}$ are called biholomorphic equivalent and $\varphi$ and $\psi$ are called biholomorphic maps.

Theorem 4.3. Let $f$ and $g$ be positive regular free holomorphic functions with $n$ and $q$ indeterminates, respectively, and let $m, l, p \geq 1$. If $F: \mathbf{D}_{f}^{m}(\mathcal{H}) \rightarrow \mathbf{D}_{g}^{l}(\mathcal{H})$ is a free biholomorphic function, then $n=q$ and its representation on $\mathbb{C}^{p}$, i.e., the map $F_{p}$ defined by

$$
\mathbb{C}^{n p^{2}} \supset \mathbf{D}_{f}^{m}\left(\mathbb{C}^{p}\right) \ni\left(\Lambda_{1}, \ldots, \Lambda_{n}\right) \mapsto F\left(\Lambda_{1}, \ldots, \Lambda_{n}\right) \in \mathbf{D}_{g}^{l}\left(\mathbb{C}^{p}\right) \subset \mathbb{C}^{q p^{2}}
$$

is a homeomorphism from $\mathbf{D}_{f}^{m}\left(\mathbb{C}^{p}\right)$ onto $\mathbf{D}_{g}^{l}\left(\mathbb{C}^{p}\right)$ and a biholomorphic function from $\operatorname{Int}\left(\mathbf{D}_{f}^{m}\left(\mathbb{C}^{p}\right)\right)$ onto $\operatorname{Int}\left(\mathbf{D}_{g}^{l}\left(\mathbb{C}^{p}\right)\right)$.

Proof. The fact that $F_{p}$ is a homeomorphism from $\mathbf{D}_{f}^{m}\left(\mathbb{C}^{p}\right)$ onto $\mathbf{D}_{g}^{l}\left(\mathbb{C}^{p}\right)$ is due to Theorem 2.2 and Corollary 2.3. Moreover, from Corollary 2.3, we also have that $F_{p}$ and $\left(F^{-1}\right)_{p}$ are holomorphic functions on $\operatorname{Int}\left(\mathbf{D}_{f}^{m}\left(\mathbb{C}^{p}\right)\right) \subset \mathbb{C}^{n p^{2}}$ and $\operatorname{Int}\left(\mathbf{D}_{g}^{l}\left(\mathbb{C}^{p}\right)\right) \subset \mathbb{C}^{q p^{2}}$, respectively. Now, since $F_{p}$ is a homeomorphism from $\mathbf{D}_{f}^{m}\left(\mathbb{C}^{p}\right)$ onto $\mathbf{D}_{g}^{l}\left(\mathbb{C}^{p}\right)$, a standard argument using Brouwer's invariance of domain theorem [4] shows that $F_{p}$ is a biholomorphic function from $\operatorname{Int}\left(\mathbf{D}_{f}^{m}\left(\mathbb{C}^{p}\right)\right)$ onto $\operatorname{Int}\left(\mathbf{D}_{g}^{l}\left(\mathbb{C}^{p}\right)\right)$ and $n=q$. This completes the proof.

We remark that under the conditions of Theorem 4.3, when $p=1$, the sets $\operatorname{Int}\left(\mathbf{D}_{f}^{m}(\mathbb{C})\right) \subset \mathbb{C}^{n}$ and $\operatorname{Int}\left(\mathbf{D}_{g}^{l}(\mathbb{C})\right) \subset \mathbb{C}^{q}$ are Reinhardt domains which contain 0. According to Sunada's result [32, we deduce that there exists a permutation $\sigma$ of the set $\{1, \ldots, n\}$ and scalars $\mu_{1}, \ldots, \mu_{n}>0$ such that the map

$$
\operatorname{Int}\left(\mathbf{D}_{f}^{m}(\mathbb{C})\right) \ni\left(z_{1}, \ldots, z_{n}\right) \mapsto\left(\mu_{1} z_{\sigma(1)}, \ldots, \mu_{n} z_{\sigma(n)}\right) \in \operatorname{Int}\left(\mathbf{D}_{g}^{l}(\mathbb{C})\right)
$$

is a biholomorphic map. It would be interesting to see if there is an analogue of Sunada's result for our noncommutative domains.

Corollary 4.4. Let $f$ and $g$ be positive regular free holomorphic functions with $n$ and $q$ indeterminates, respectively, and let $m, l, p \geq 1$. If $n \neq q$ or there is $p \in\{1,2, \ldots\}$ such that $\operatorname{Int}\left(\mathbf{D}_{f}^{m}\left(\mathbb{C}^{p}\right)\right)$ is not biholomorphic equivalent to $\operatorname{Int}\left(\mathbf{D}_{g}^{l}\left(\mathbb{C}^{p}\right)\right)$, then the noncommutative domains $\mathbf{D}_{f}^{m}(\mathcal{H})$ and $\mathbf{D}_{g}^{l}(\mathcal{H})$ are not free biholomorphic equivalent. 
Let $f=\sum_{|\alpha| \geq 1} a_{\alpha} X_{\alpha}$ be a positive regular free holomorphic function and fix $c_{1}, \ldots, c_{n}>0$. Define $g:=\sum_{|\alpha| \geq 1} d_{\alpha} X_{\alpha}$ by setting $d_{\alpha}:=\frac{1}{c^{2 \alpha}} a_{\alpha}$ if $|\alpha| \geq 1$, where $c:=\left(c_{1}, \ldots, c_{n}\right)$ and $c^{\alpha}:=c_{i_{1}} \cdots c_{i_{k}}$ when $\alpha=g_{i_{1}} \cdots g_{i_{k}} \in \mathbb{F}_{n}^{+}$. It is easy to see that $g$ is also a positive regular free holomorphic function. Note that the noncommutative domains $\mathbf{D}_{f}^{m}(\mathcal{H})$ and $\mathbf{D}_{g}^{m}(\mathcal{H})$ are free biholomorphic equivalent. Indeed, the map $\Lambda: \mathbf{D}_{f}^{m}(\mathcal{H}) \rightarrow \mathbf{D}_{g}^{m}(\mathcal{H})$ defined by $\Lambda\left(X_{1}, \ldots, X_{n}\right):=\left(c_{1} X_{1}, \ldots, c_{n} X_{n}\right)$ is a free biholomorphic function. Therefore, by rescaling variables, we obtain free biholomorphic equivalent domains.

In what follows, we characterize the set $B i h_{0}\left(\mathbf{D}_{f}^{m}, \mathbf{D}_{g}^{l}\right)$ of all free biholomorphic functions $F: \mathbf{D}_{f}^{m}(\mathcal{H}) \rightarrow$ $\mathbf{D}_{g}^{l}(\mathcal{H})$ with $F(0)=0$. We use the notation $\left(W_{1}^{(f)}, \ldots, W_{n}^{(f)}\right)$ for the universal model associated with the noncommutative domain $\mathbf{D}_{f}^{m}$. Here is the main result of this section.

Theorem 4.5. Let $f$ and $g$ be positive regular free holomorphic functions with $n$ and $q$ indeterminates, respectively, and let $m, l \geq 1$. A map $F: \mathbf{D}_{f}^{m}(\mathcal{H}) \rightarrow \mathbf{D}_{g}^{l}(\mathcal{H})$ is a free biholomorphic function with $F(0)=0$ if and only if $n=q$ and $F$ has the form

$$
F\left(X_{1}, \ldots, X_{n}\right)=\left[X_{1}, \ldots, X_{n}\right] U, \quad\left(X_{1}, \ldots, X_{n}\right) \in \mathbf{D}_{f}^{m}(\mathcal{H}),
$$

where $U$ is an invertible bounded linear operator on $\mathbb{C}^{n}$ such that

$$
\left[W_{1}^{(f)}, \ldots, W_{n}^{(f)}\right] U \in \mathbf{D}_{g}^{l}\left(F^{2}\left(H_{n}\right)\right) \quad \text { and } \quad\left[W_{1}^{(g)}, \ldots, W_{n}^{(g)}\right] U^{-1} \in \mathbf{D}_{f}^{m}\left(F^{2}\left(H_{n}\right)\right) .
$$

Proof. Assume that $F: \mathbf{D}_{f}^{m}(\mathcal{H}) \rightarrow \mathbf{D}_{g}^{l}(\mathcal{H})$ is a free biholomorphic function with $F(0)=0$. According to Theorem 4.3, we must have $n=q$. Then $F$ has a representation $F=\left(F_{1}, \ldots, F_{n}\right)$, where $F_{j}$ is a power series with scalar coefficients, having the representation

$$
F_{j}\left(Z_{1}, \ldots, Z_{n}\right)=\sum_{k=1}^{n} a_{k j} Z_{k}+\Psi_{2}^{(j)}\left(Z_{1}, \ldots, Z_{n}\right)
$$

and $\Psi_{2}^{(j)}$ is a power series of the form $\Psi_{2}^{(j)}\left(Z_{1}, \ldots, Z_{n}\right)=\sum_{p=2}^{\infty} \sum_{|\alpha|=p} a_{\alpha}^{(j)} Z_{\alpha}$. It is easy to see that if $\left(N_{1}, \ldots, N_{n}\right) \in \mathbf{D}_{f, \text { nil }}^{m}(\mathcal{H})$, then $F\left(N_{1}, \ldots, N_{n}\right) \in \mathbf{D}_{g, \text { nil }}^{l}(\mathcal{H})$. Similarly, one can show that $F^{-1}\left(\mathbf{D}_{g, \text { nil }}^{l}(\mathcal{H})\right) \subseteq \mathbf{D}_{f, \text { nil }}^{m}(\mathcal{H})$. Therefore, we have $F\left(\mathbf{D}_{f, \text { nil }}^{m}(\mathcal{H})\right)=\mathbf{D}_{g, \text { nil }}^{l}(\mathcal{H})$. Applying Theorem 4.2 , we deduce that $F\left(Z_{1}, \ldots, Z_{n}\right)=\left[Z_{1}, \ldots, Z_{n}\right] U$, where $U$ is an invertible bounded linear operator on $\mathbb{C}^{n}$. Since $F: \mathbf{D}_{f}^{m}(\mathcal{H}) \rightarrow \mathbf{D}_{g}^{l}(\mathcal{H})$ is a free biholomorphic function, $\left(r W_{1}^{(f)}, \ldots, r W_{n}^{(f)}\right) \in \mathbf{D}_{f, \text { rad }}^{m}(\mathcal{H})$, and $\left(r W_{1}^{(g)}, \ldots, r W_{n}^{(g)}\right) \in \mathbf{D}_{g, \text { rad }}^{l}(\mathcal{H})$, we deduce that

$$
\left[r W_{1}^{(f)}, \ldots, r W_{n}^{(f)}\right] U \in \mathbf{D}_{g}^{l}\left(F^{2}\left(H_{n}\right)\right) \quad \text { and } \quad\left[r W_{1}^{(g)}, \ldots, r W_{n}^{(g)}\right] U^{-1} \in \mathbf{D}_{f}^{m}\left(F^{2}\left(H_{n}\right)\right)
$$

for any $r \in[0,1)$. Since the domains $\mathbf{D}_{g}^{l}\left(F^{2}\left(H_{n}\right)\right)$ and $\mathbf{D}_{f}^{m}\left(F^{2}\left(H_{n}\right)\right)$ are closed in the operator norm topology, and taking $r \rightarrow 1$, we obtain the assertion of the theorem.

Conversely, assume that $F$ has the form

$$
F\left(X_{1}, \ldots, X_{n}\right)=\left[X_{1}, \ldots, X_{n}\right] U, \quad\left(X_{1}, \ldots, X_{n}\right) \in \mathbf{D}_{f}^{m}(\mathcal{H}),
$$

where $U$ is an invertible bounded linear operator on $\mathbb{C}^{n}$ such that

$$
\left[W_{1}^{(f)}, \ldots, W_{n}^{(f)}\right] U \in \mathbf{D}_{g}^{l}\left(F^{2}\left(H_{n}\right)\right) \quad \text { and } \quad\left[W_{1}^{(g)}, \ldots, W_{n}^{(g)}\right] U^{-1} \in \mathbf{D}_{f}^{m}\left(F^{2}\left(H_{n}\right)\right) .
$$

Define the free holomorphic function $G$ by setting

$$
G\left(Y_{1}, \ldots, Y_{n}\right)=\left[Y_{1}, \ldots, Y_{n}\right] U^{-1}, \quad\left(Y_{1}, \ldots, Y_{n}\right) \in \mathbf{D}_{g}^{l}(\mathcal{H})
$$

It is clear that $F$ and $G$ are continuous functions in the operator norm topology. Moreover, note that $\left.F\right|_{\mathbf{D}_{f, \text { rad }}^{m}(\mathcal{H})}$ and $\left.F^{-1}\right|_{\mathbf{D}_{g, \text { rad }}^{l}(\mathcal{H})}$ are free holomorphic functions on $\mathbf{D}_{f, \text { rad }}^{m}(\mathcal{H})$ and $\mathbf{D}_{g, \text { rad }}^{l}(\mathcal{H})$, respectively. Since $F \circ G=G \circ F=$ id, it remains to show that

$$
F\left(\mathbf{D}_{f}^{m}(\mathcal{H})\right) \subseteq \mathbf{D}_{g}^{l}(\mathcal{H}) \quad \text { and } \quad G\left(\mathbf{D}_{g}^{l}(\mathcal{H})\right) \subseteq \mathbf{D}_{f}^{m}(\mathcal{H})
$$

To this end, let $\left(X_{1}, \ldots, X_{n}\right) \in \mathbf{D}_{f}^{m}(\mathcal{H})$ and fix $r \in[0,1)$. Then $\left(r X_{1}, \ldots, r X_{n}\right) \in \mathbf{D}_{f, \text { rad }}^{m}(\mathcal{H}) \subset \mathbf{D}_{f, \text { pure }}^{m}(\mathcal{H})$ and, due to the dilation theorem from [24] there is a separable infinite dimensional Hilbert space $\mathcal{M}$ such that

$$
r X_{i}^{*}=\left.\left(W_{i}^{(f)^{*}} \otimes I_{\mathcal{M}}\right)\right|_{\mathcal{H}}, \quad i=1, \ldots, n,
$$


where $\mathcal{H}$ is identified with a co-invariant subspace of $F^{2}\left(H_{n}\right) \otimes \mathcal{M}$ under the operators $W_{1}^{(f)^{*}} \otimes I_{\mathcal{M}}, \ldots$, $W_{n}^{(f)^{*}} \otimes I_{\mathcal{M}}$. Let $U:=\left[a_{j i}\right]$ be the matrix representation of the invertible operator from relation (4.7). Setting $L_{i}:=\sum_{k=1}^{n} a_{k i} W_{k}^{(f)}, i=1, \ldots, n$, the first part of relation (4.7) implies

$$
\left(i d-\Phi_{g, \mathbf{L}}\right)^{l}\left(I_{F^{2}\left(H_{n}\right) \otimes \mathcal{M}}\right)=\sum_{p=0}^{l}(-1)^{p}\left(\begin{array}{l}
l \\
p
\end{array}\right) \Phi_{g, \mathbf{L}}^{p}\left(I_{\left.F^{2}\left(H_{n}\right) \otimes \mathcal{M}\right) \geq 0,}\right.
$$

where $\mathbf{L}:=\left(L_{1} \otimes I_{\mathcal{M}}, \ldots, L_{n} \otimes I_{\mathcal{M}}\right)$. Note that each $\Phi_{g, \mathbf{L}}^{p}\left(I_{F^{2}\left(H_{n}\right) \otimes \mathcal{M}}\right)$ is given by a series which is convergent in the weak operator topology and, due to condition (4.8), we deduce that

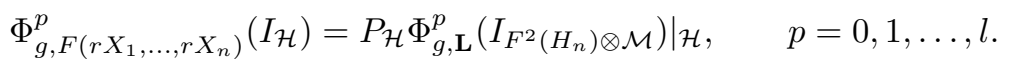

Combining this relation with inequality (4.9), we obtain $\left(i d-\Phi_{g, F\left(r X_{1}, \ldots, r X_{n}\right)}\right)^{l}\left(I_{\mathcal{H}}\right) \geq 0$, which shows that $F\left(r X_{1}, \ldots, r X_{n}\right) \in \mathbf{D}_{g}^{l}(\mathcal{H})$ for any $r \in[0,1)$. Since $\mathbf{D}_{g}^{l}(\mathcal{H})$ is closed in the operator norm topology and $F\left(r X_{1}, \ldots, r X_{n}\right)=r\left[X_{1}, \ldots, X_{n}\right] U$, we deduce that $\left[X_{1}, \ldots, X_{n}\right] U \in \mathbf{D}_{g}^{l}(\mathcal{H})$ for any $n$-tuple $\left(X_{1}, \ldots, X_{n}\right) \in \mathbf{D}_{f}^{m}(\mathcal{H})$. This proves that $F\left(\mathbf{D}_{f}^{m}(\mathcal{H})\right) \subseteq \mathbf{D}_{g}^{l}(\mathcal{H})$. Similarly, one can prove the inclusion $G\left(\mathbf{D}_{g}^{l}(\mathcal{H})\right) \subseteq \mathbf{D}_{f}^{m}(\mathcal{H})$. This completes the proof.

We denote by $\operatorname{Aut}\left(\mathbf{D}_{f}^{m}\right):=\operatorname{Bih}\left(\mathbf{D}_{f}^{m}, \mathbf{D}_{f}^{m}\right)$ the set of all free biholomorphic functions of $\mathbf{D}_{f}^{m}(\mathcal{H})$. Due to Theorem 3.3. $A u t\left(\mathbf{D}_{f}^{m}\right)$ is a group with respect to the composition of free holomorphic functions. As a consequence of Theorem 4.5, we obtain the following characterization of $A u t_{0}\left(\mathbf{D}_{f}^{m}\right)$, the subgroup of all free holomorphic automorphisms of $\mathbf{D}_{f}^{m}(\mathcal{H})$ that fix the origin.

Corollary 4.6. Let $f$ be a positive regular free holomorphic function with $n$ indeterminates and let $m \geq 1$. A map $\Psi: \mathbf{D}_{f}^{m}(\mathcal{H}) \rightarrow \mathbf{D}_{f}^{m}(\mathcal{H})$ is a free holomorphic automorphism with $\Psi(0)=0$ if and only if it has the form

$$
\Psi\left(X_{1}, \ldots, X_{n}\right)=\left[X_{1}, \ldots, X_{n}\right] U, \quad\left(X_{1}, \ldots, X_{n}\right) \in \mathbf{D}_{f}^{m}(\mathcal{H}),
$$

where $U$ is an invertible operator on $\mathbb{C}^{n}$ such that

$$
\left[W_{1}, \ldots, W_{n}\right] U \in \mathbf{D}_{f}^{m}\left(F^{2}\left(H_{n}\right)\right) \quad \text { and } \quad\left[W_{1}, \ldots, W_{n}\right] U^{-1} \in \mathbf{D}_{f}^{m}\left(F^{2}\left(H_{n}\right)\right),
$$

and $\left(W_{1}, \ldots, W_{n}\right)$ is the universal model associated with the noncommutative domain $\mathbf{D}_{f}^{m}$.

Now, we can characterize the unit ball of $B(\mathcal{H})^{n}$ among the noncommutative domains $\mathbf{D}_{f}^{m}(\mathcal{H})$, up to free biholomorphisms.

Corollary 4.7. Let $g$ be a positive regular free holomorphic function with $q$ indeterminates and let $l \geq 1$. Then the noncommutative domain $\mathbf{D}_{g}^{l}(\mathcal{H})$ is biholomorphic equivalent to the unit ball $\left[B(\mathcal{H})^{n}\right]_{1}$ if and only if $q=n$ and there is an invertible bounded linear operator $U \in B\left(\mathbb{C}^{n}\right)$ such that

$$
\left[S_{1}, \ldots, S_{n}\right] U \in \mathbf{D}_{g}^{l}\left(F^{2}\left(H_{n}\right)\right) \quad \text { and } \quad\left[W_{1}^{(g)}, \ldots, W_{n}^{(g)}\right] U^{-1} \in\left[B(\mathcal{H})^{n}\right]_{1}^{-},
$$

where $S_{1}, \ldots, S_{n}$ are the left creation operators on the full Fock space $F^{2}\left(H_{n}\right)$.

Proof. Let $p:=X_{1}+\cdots+X_{n}$ and recall that $\mathbf{D}_{p}^{1}(\mathcal{H})=\left[B(\mathcal{H})^{n}\right]_{1}^{-}$. Assume that $F: \mathbf{D}_{g}^{l}(\mathcal{H}) \rightarrow \mathbf{D}_{p}^{1}(\mathcal{H})$ is a free biholomorphic function. Due to Theorem 4.3, $n=q$ and the representation of $F$ on $\mathbb{C}$, i.e., the map $F_{1}$ defined by

$$
\mathbb{C}^{n} \supset \mathbf{D}_{p}^{1}(\mathbb{C}) \ni\left(\lambda_{1}, \ldots, \lambda_{n}\right) \mapsto F\left(\lambda_{1}, \ldots, \lambda_{n}\right) \in \mathbf{D}_{g}^{l}(\mathbb{C}) \subset \mathbb{C}^{n}
$$

is a homeomorphism from $\mathbf{D}_{p}^{1}(\mathbb{C})$ onto $\mathbf{D}_{g}^{l}(\mathbb{C})$ and a biholomorphic function from $\operatorname{Int}\left(\mathbf{D}_{p}^{1}(\mathbb{C})\right)=\left[B(\mathcal{H})^{n}\right]_{1}$ onto $\operatorname{Int}\left(\mathbf{D}_{g}^{l}(\mathbb{C})\right)$. Consequently, $z_{0}:=F(0) \in \mathbb{B}_{n}$, the open unit ball of $\mathbb{C}^{n}$. According to Theorem 2.3 from [30], there is a free holomorphic automorphism $\Psi_{z_{0}}$ of the open unit ball $\left[B(\mathcal{H})^{n}\right]_{1}$ such that $\Psi_{z_{0}}\left(z_{0}\right)=0$. Moreover, $\Psi_{z_{0}}$ has a continuous extension to the closed ball $\left[B(\mathcal{H})^{n}\right]_{1}^{-}$which is a homeomorphism of $\left[B(\mathcal{H})^{n}\right]_{1}^{-}$. Then, according to Theorem 3.3, the composition $\Psi_{z_{0}} \circ F: \mathbf{D}_{g}^{l}(\mathcal{H}) \rightarrow \mathbf{D}_{p}^{1}(\mathcal{H})$ is a free biholomorphic function with the property that $\left(\Psi_{z_{0}} \circ F\right)(0)=0$. Applying now Theorem 4.5 , we find an invertible bounded linear operator $U \in B\left(\mathbb{C}^{n}\right)$ such that

$$
\left[S_{1}, \ldots, S_{n}\right] U \in \mathbf{D}_{g}^{l}\left(F^{2}\left(H_{n}\right)\right) \quad \text { and } \quad\left[W_{1}^{(g)}, \ldots, W_{n}^{(g)}\right] U^{-1} \in\left[B(\mathcal{H})^{n}\right]_{1}^{-},
$$


and such that $\left(\Psi_{z_{0}} \circ F\right)\left(X_{1}, \ldots, X_{n}\right)=\left[X_{1}, \ldots, X_{n}\right] U$ for all $\left(X_{1}, \ldots, X_{n}\right) \in \mathbf{D}_{g}^{l}(\mathcal{H})$. The converse follows easily from Theorem 4.5 .

We remark that in the particular case when $l=1$ we will get a more precise result in the next section (see Corollary 5.3).

In what follows we use again the interaction between the theory of functions in several complex variables and our noncommutative theory to obtain some results on the classification of the noncommutative domains $\mathbf{D}_{f}^{m}(\mathcal{H}), m \geq 1$.

Theorem 4.8. Let $f$ and $g$ be positive regular free holomorphic functions with $n$ indeterminates and let $m, l \geq 1$. Assume that there is $p^{\prime} \in\{1,2, \ldots\}$ such that the domains $\operatorname{Int}\left(\mathbf{D}_{f}^{m}\left(\mathbb{C}^{p^{\prime}}\right)\right)$ and $\operatorname{Int}\left(\mathbf{D}_{g}^{l}\left(\mathbb{C}^{p^{\prime}}\right)\right)$ are linearly equivalent and all the automorphisms of $\operatorname{Int}\left(\mathbf{D}_{f}^{m}\left(\mathbb{C}^{p^{\prime}}\right)\right)$ fix the origin.

Then the noncommutative domains $\mathbf{D}_{f}^{m}(\mathcal{H})$ and $\mathbf{D}_{g}^{l}(\mathcal{H})$ are free biholomorphic equivalent if and only if there is an invertible bounded linear operator $U \in B\left(\mathbb{C}^{n}\right)$ such that

$$
\left[W_{1}^{(f)}, \ldots, W_{n}^{(f)}\right] U \in \mathbf{D}_{g}^{l}\left(F^{2}\left(H_{n}\right)\right) \quad \text { and } \quad\left[W_{1}^{(g)}, \ldots, W_{n}^{(g)}\right] U^{-1} \in \mathbf{D}_{f}^{m}\left(F^{2}\left(H_{n}\right)\right) .
$$

Proof. Since the domains $\operatorname{Int}\left(\mathbf{D}_{f}^{m}\left(\mathbb{C}^{p^{\prime}}\right)\right)$ and $\operatorname{Int}\left(\mathbf{D}_{g}^{l}\left(\mathbb{C}^{p^{\prime}}\right)\right)$ are linearly equivalent, there is a biholomorphic function $\varphi: \operatorname{Int}\left(\mathbf{D}_{f}^{m}\left(\mathbb{C}^{p^{\prime}}\right)\right) \rightarrow \operatorname{Int}\left(\mathbf{D}_{g}^{l}\left(\mathbb{C}^{p^{\prime}}\right)\right)$ such that $\varphi(0)=0$. Suppose that $F: \mathbf{D}_{f}^{m}(\mathcal{H}) \rightarrow \mathbf{D}_{g}^{l}(\mathcal{H})$ is a free biholomorphic function. According to Theorem 4.3 $n=q$ and the representation of $F$ on $\mathbb{C}^{p^{\prime}}$, i.e., the map $F_{p^{\prime}}$ defined by

$$
\mathbb{C}^{n p^{\prime 2}} \supset \mathbf{D}_{f}^{m}\left(\mathbb{C}^{p^{\prime}}\right) \ni\left(\Lambda_{1}, \ldots, \Lambda_{n}\right) \mapsto F\left(\Lambda_{1}, \ldots, \Lambda_{n}\right) \in \mathbf{D}_{g}^{l}\left(\mathbb{C}^{p^{\prime}}\right) \subset \mathbb{C}^{n p^{\prime 2}}
$$

is a biholomorphic function from $\operatorname{Int}\left(\mathbf{D}_{f}^{m}\left(\mathbb{C}^{p^{\prime}}\right)\right)$ onto $\operatorname{Int}\left(\mathbf{D}_{g}^{l}\left(\mathbb{C}^{p^{\prime}}\right)\right)$. Consequently, $\varphi^{-1} \circ F_{p^{\prime}}$ is an automorphism of $\operatorname{Int}\left(\mathbf{D}_{f}^{m}\left(\mathbb{C}^{p^{\prime}}\right)\right)$. Due to the hypothesis, we have $\left(\varphi^{-1} \circ F_{p^{\prime}}\right)(0)=0$. Therefore, $F_{p^{\prime}}(0)=0$, which obviously implies $F(0)=0$. Applying now Theorem 4.5 the result follows. The converse is due to the same theorem. The proof is complete.

Using Thullen characterization of domains in $\mathbb{C}^{2}$ with non-compact automorphism group (34) and Theorem 4.5. we can obtain the following classification result for noncommutative domains generated by positive regular free holomorphic functions in 2 indeterminates. We recall that Thullen proved that if a bounded Reinhardt domain in $\mathbb{C}^{2}$ has a biholomorphic map that does not fix the origin, then the domain is linearly equivalent to one of the following: polydisc, unit ball, or the so-called Thullen domain. Combining this result with Theorem 4.8, we obtain the following consequence.

Corollary 4.9. Let $f$ and $g$ be positive regular free holomorphic functions with 2 indeterminates and let $m, l \geq 1$. Assume that the Reinhardt domains $\operatorname{Int}\left(\mathbf{D}_{f}^{m}(\mathbb{C})\right)$ and $\operatorname{Int}\left(\mathbf{D}_{g}^{l}(\mathbb{C})\right)$ are linearly equivalent but they are not linearly equivalent to either the polydisc, the unit ball, or any Thullen domain in $\mathbb{C}^{2}$.

Then the noncommutative domains $\mathbf{D}_{f}^{m}(\mathcal{H})$ and $\mathbf{D}_{g}^{l}(\mathcal{H})$ are free biholomorphic equivalent if and only if there is an invertible bounded linear operator $U \in B\left(\mathbb{C}^{2}\right)$ such that

$$
\left[W_{1}^{(f)}, W_{2}^{(f)}\right] U \in \mathbf{D}_{g}^{l}\left(F^{2}\left(H_{2}\right)\right) \quad \text { and } \quad\left[W_{1}^{(g)}, W_{2}^{(g)}\right] U^{-1} \in \mathbf{D}_{f}^{m}\left(F^{2}\left(H_{2}\right)\right) .
$$

\section{FREE BIHOLOMORPHIC CLASSIFICATION OF NONCOMMUTATIVE DOMAINS}

The main result of this section shows that the free biholomorphic classification of the noncommutative domains $\mathbf{D}_{f}^{m}(\mathcal{H}) \subset B(\mathcal{H})^{n}$ is the same as the classification of the corresponding noncommutative domain algebras $\mathcal{A}_{n}\left(\mathbf{D}_{f}^{m}\right) \simeq A\left(\mathbf{D}_{f, \text { rad }}^{m}\right)$. Combining this result with the Cartan type results from Section 4, we provide several consequences concerning the free biholomorphic classification.

Let $f$ and $g$ be positive regular free holomorphic functions with $n$ and $q$ indeterminates, respectively, and let $m, l \geq 1$. According to Theorem 2.2, there is a completely isometric isomorphism

$$
A\left(\mathbf{D}_{f, \mathrm{rad}}^{m}\right) \ni \chi \mapsto \tilde{\chi}:=\lim _{r \rightarrow 1} \chi\left(r W_{1}, \ldots, r W_{n}\right) \in \mathcal{A}_{n}\left(\mathbf{D}_{f}^{m}\right),
$$


where the limit is in the operator norm topology, whose inverse is the noncommutative Berezin transform B, i.e., $\chi(X)=\mathbf{B}_{X}[\widetilde{\chi}]$ for any $X \in \mathbf{D}_{f, \text { rad }}^{m}(\mathcal{H})$. If $\Phi: A\left(\mathbf{D}_{f, \text { rad }}^{m}\right) \rightarrow A\left(\mathbf{D}_{g, \text { rad }}^{l}\right)$ is a unital algebra homomorphism, it induces a unique unital homomorphism $\widehat{\Phi}: \mathcal{A}_{n}\left(\mathbf{D}_{f}^{m}\right) \rightarrow \mathcal{A}_{q}\left(\mathbf{D}_{g}^{l}\right)$ such that the diagram

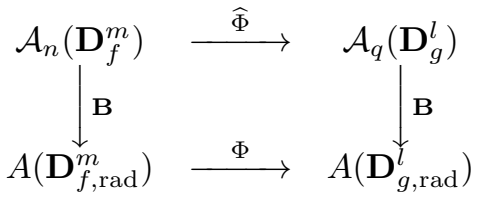

is commutative, i.e., $\Phi \mathbf{B}=\mathbf{B} \widehat{\Phi}$, where $\mathbf{B}$ is the appropriate noncommutative Berezin transform on the noncommutative domain algebra $\mathcal{A}_{n}\left(\mathbf{D}_{f}^{m}\right)$ or $\mathcal{A}_{q}\left(\mathbf{D}_{g}^{l}\right)$ (see relation (1.6)). The homomorphisms $\Phi$ and $\widehat{\Phi}$ uniquely determine each other by the formulas:

$$
\begin{aligned}
{[\Phi(\chi)](X) } & =\mathbf{B}_{X}[\widehat{\Phi}(\widetilde{\chi})], \quad \chi \in A\left(\mathbf{D}_{f, \mathrm{rad}}^{m}\right), \quad X \in \mathbf{D}_{g, \mathrm{rad}}^{l}(\mathcal{H}), \quad \text { and } \\
\widehat{\Phi}(\widetilde{\chi}) & =\widetilde{\Phi(\chi)}, \quad \tilde{\chi} \in \mathcal{A}_{n}\left(\mathbf{D}_{f}^{m}\right) .
\end{aligned}
$$

We recall that $\operatorname{Bih}\left(\mathbf{D}_{f}^{m}, \mathbf{D}_{g}^{l}\right)$ stands for the set of all biholomorphic functions from $\mathbf{D}_{f}^{m}(\mathcal{H})$ onto $\mathbf{D}_{g}^{l}(\mathcal{H})$, i.e., all homeomorphisms $\varphi: \mathbf{D}_{f}^{m}(\mathcal{H}) \rightarrow \mathbf{D}_{g}^{l}(\mathcal{H})$ with the property that $\left.\varphi\right|_{\mathbf{D}_{f, \text { rad }}^{m}(\mathcal{H})}$ and $\left.\varphi^{-1}\right|_{\mathbf{D}_{g, \text { rad }}^{l}(\mathcal{H})}$ are free holomorphic functions. Let $\Phi$ be a unital completely isometric isomorphism from the noncommutative domain algebra $A\left(\mathbf{D}_{f, \text { rad }}^{m}\right)$ onto $A\left(\mathbf{D}_{g, \text { rad }}^{l}\right)$. Consider the closed operator systems in $B\left(F^{2}\left(H_{n}\right)\right)$,

$$
\mathcal{S}_{f}:=\overline{\operatorname{span}}\left\{W_{\alpha}^{(f)} W_{\beta}^{(f)^{*}} ; \alpha, \beta \in \mathbb{F}_{n}^{+}\right\} \quad \text { and } \quad \mathcal{S}_{g}:=\overline{\operatorname{span}}\left\{W_{\alpha}^{(g)} W_{\beta}^{(g)^{*}} ; \alpha, \beta \in \mathbb{F}_{q}^{+}\right\},
$$

where $\left(W_{1}^{(f)}, \ldots, W_{n}^{(f)}\right)$ and $\left(W_{1}^{(g)}, \ldots, W_{q}^{(g)}\right)$ are the universal models of the noncommutative domains $\mathbf{D}_{f}^{m}$ and $\mathbf{D}_{g}^{l}$, respectively. We say that $\Phi$ has completely contractive hereditary extension if the induced isomorphism $\widehat{\Phi}: \mathcal{A}_{n}\left(\mathbf{D}_{f}^{m}\right) \rightarrow \mathcal{A}_{q}\left(\mathbf{D}_{g}^{l}\right)$ has the property that the linear maps $\widehat{\Phi}_{*}: \mathcal{S}_{f} \rightarrow \mathcal{S}_{g}$ and $\left(\widehat{\Phi}^{-1}\right)_{*}$ : $\mathcal{S}_{g} \rightarrow \mathcal{S}_{f}$ defined by

$$
\widehat{\Phi}_{*}\left(W_{\alpha}^{(f)} W_{\beta}^{(f)^{*}}\right):=\widehat{\Phi}\left(W_{\alpha}^{(f)}\right) \widehat{\Phi}\left(W_{\beta}^{(f)}\right)^{*}, \quad \alpha, \beta \in \mathbb{F}_{n}^{+},
$$

and

$$
\left.\left(\widehat{\Phi}^{-1}\right)_{*}\left(W_{\alpha}^{(g)} W_{\beta}^{(g)}\right)^{*}\right):=\widehat{\Phi}^{-1}\left(W_{\alpha}^{(g)}\right) \widehat{\Phi}^{-1}\left(W_{\beta}^{(g)}\right)^{*}, \quad \alpha, \beta \in \mathbb{F}_{q}^{+},
$$

respectively, are completely contractive. Using an approximation argument, one can easily show that $\widehat{\Phi}_{*} \circ\left(\widehat{\Phi}^{-1}\right)_{*}=i d_{\mathcal{S}_{g}}$ and $\left(\widehat{\Phi}^{-1}\right)_{*} \circ \widehat{\Phi}_{*}=i d_{\mathcal{S}_{f}}$. Consequently, the map $\widehat{\Phi}_{*}: \mathcal{S}_{f} \rightarrow \mathcal{S}_{g}$ is a unital completely isometric linear isomorphism between operator systems, which extends the completely isometric isomorphism $\widehat{\Phi}: \mathcal{A}_{n}\left(\mathbf{D}_{f}^{m}\right) \rightarrow \mathcal{A}_{q}\left(\mathbf{D}_{g}^{l}\right)$.

Now, we are ready to prove the main result of this section. We show that the free biholomorphic classification of the domains $\mathbf{D}_{f}^{m}(\mathcal{H})$ is the same as the classification, up to unital completely isometric isomorphisms having completely contractive hereditary extension, of the corresponding noncommutative domain algebras $A\left(\mathbf{D}_{f, \text { rad }}^{m}\right) \simeq \mathcal{A}_{n}\left(\mathbf{D}_{f}^{m}\right)$.

Theorem 5.1. Let $f$ and $g$ be positive regular free holomorphic functions with $n$ and $q$ indeterminates, respectively, and let $m, l \geq 1$. Then the following statements are equivalent:

(i) $\Psi: A\left(\mathbf{D}_{f, \text { rad }}^{m}\right) \rightarrow A\left(\mathbf{D}_{g, \text { rad }}^{l}\right)$ is a unital completely isometric isomorphism with completely contractive hereditary extension;

(ii) there is $\varphi \in \operatorname{Bih}\left(\mathbf{D}_{g}^{l}, \mathbf{D}_{f}^{m}\right)$ such that

$$
\Psi(\chi)=\chi \circ \varphi, \quad \chi \in A\left(\mathbf{D}_{f, \mathrm{rad}}^{m}\right) .
$$

In this case, $\widehat{\Psi}(\widetilde{\chi})=\mathcal{B}_{\widetilde{\varphi}}[\widetilde{\chi}], \tilde{\chi} \in \mathcal{A}_{n}\left(\mathbf{D}_{f}^{m}\right)$, where $\mathcal{B}_{\widetilde{\varphi}}$ is the noncommutative Berezin transform at $\widetilde{\varphi}$. In the particular case when $m=l=1$, any unital completely isometric isomorphism has completely contractive hereditary extension. 
Proof. Let $\sum_{\alpha \in \mathbb{F}_{n}^{+},|\alpha| \geq 1} a_{\alpha} X_{\alpha}$ be the representation of $f$. First we prove that $(i) \Longrightarrow(i i)$. Assume that $\Psi$ is a unital completely isometric isomorphism. Then, the induced map $\widehat{\Psi}: \mathcal{A}_{n}\left(\mathbf{D}_{f}^{m}\right) \rightarrow \mathcal{A}_{q}\left(\mathbf{D}_{g}^{l}\right)$ is a completely isometric isomorphism. Denote

$$
\widetilde{\varphi}_{i}:=\widehat{\Psi}\left(W_{i}^{(f)}\right) \in \mathcal{A}_{q}\left(\mathbf{D}_{g}^{l}\right), \quad i=1, \ldots, n,
$$

where $W^{(f)}:=\left(W_{1}^{(f)}, \ldots, W_{n}^{(f)}\right) \in \mathbf{D}_{f}^{m}\left(F^{2}\left(H_{n}\right)\right)$ is the universal model of the noncommutative domain $\mathbf{D}_{f}^{m}$. Since $\Phi_{f, W^{(f)}}(I) \leq I$, we have $\Phi_{f, r W^{(f)}}(I)=\sum_{k=1}^{\infty} \sum_{|\alpha|=k} a_{\alpha} r^{|\alpha|} W_{\alpha}^{(f)} W_{\alpha}^{(f)}{ }^{*} \leq I$ and $\Phi_{f, r W^{(f)}}(I) \in$ $\mathcal{S}_{f}$ for any $r \in[0,1)$. Using relation (5.1) and the fact that $\widehat{\Psi}$ is a completely isometric homomorphism, we deduce that $0 \leq \Phi_{f, r \widetilde{\varphi}}(I) \leq I$ for $r \in[0,1)$, which proves that $r \widetilde{\varphi}:=\left(r \widetilde{\varphi}_{1}, \ldots, r \widetilde{\varphi}_{n}\right) \in \mathbf{D}_{f}^{1}\left(F^{2}\left(H_{n}\right)\right)$. Since $\mathbf{D}_{f}^{1}\left(F^{2}\left(H_{n}\right)\right)$ is closed in the operator norm topology, we deduce that $\widetilde{\varphi} \in \mathbf{D}_{f}^{1}\left(F^{2}\left(H_{n}\right)\right)$.

We recall (see Corollary 1.5 from [24]) that if $\psi$ is a positive linear map on $B(\mathcal{H})$ such that $\psi(I) \leq I$ and $(i d-\psi)^{m}(I) \geq 0$ for some $m \in \mathbb{N}$, then

$$
0 \leq(i d-\psi)^{m}(I) \leq(i d-\psi)^{m-1}(I) \leq \cdots \leq(i d-\psi)(I) \leq I .
$$

Since $\mathbf{D}_{f}^{m}$ is a starlike domain, $\left(r W_{1}, \ldots, r W_{n}\right) \in \mathbf{D}_{f}^{m}\left(F^{2}\left(H_{n}\right)\right)$, i.e., $\left(i d-\Phi_{f, r W^{(f)}}\right)^{m}(I) \geq 0$. Using the above-mentioned result in our setting, we have

$$
0 \leq\left(i d-\Phi_{f, r W^{(f)}}\right)^{s}(I)=\sum_{p=0}^{s}(-1)^{p}\left(\begin{array}{l}
s \\
p
\end{array}\right) \Phi_{f, r W^{(f)}}^{p}(I)=I-\sum_{p=1}^{s}(-1)^{p+1}\left(\begin{array}{l}
s \\
p
\end{array}\right) \Phi_{f, r W^{(f)}}^{p}(I) \leq I
$$

for any $s=1, \ldots, m$, which is equivalent to

$$
0 \leq \sum_{p=1}^{s}(-1)^{p+1}\left(\begin{array}{c}
s \\
p
\end{array}\right) \Phi_{f, r W^{(f)}}^{p}(I) \leq I, \quad s=1, \ldots, m .
$$

Since

$$
\Phi_{f, r W^{(f)}}^{p}(I)=\sum_{k=1}^{\infty} \sum_{|\alpha|=k} a_{\alpha} r^{|\alpha|} W_{\alpha}^{(f)} \Phi_{f, r W^{(f)}}^{p-1}(I) W_{\alpha}^{(f)^{*}}
$$

and $\left\|\Phi_{f, r W^{(f)}}^{p}(I)\right\| \leq 1$ for any $p \in \mathbb{N}$, it is clear that $\Phi_{f, r W^{(f)}}^{p}(I) \in \mathcal{S}_{f}:=\overline{\operatorname{span}}\left\{W_{\alpha}^{(f)} W_{\beta}^{(f)^{*}} ; \alpha, \beta \in \mathbb{F}_{n}^{+}\right\}$. Assume now that $\Psi: A\left(\mathbf{D}_{f, \text { rad }}^{m}\right) \rightarrow A\left(\mathbf{D}_{g \text {,rad }}^{l}\right)$ satisfies condition (i). Then, due to the considerations above, relation (5.2) holds and implies

$$
\left\|\sum_{p=1}^{s}(-1)^{p+1}\left(\begin{array}{l}
s \\
p
\end{array}\right) \Phi_{f, r W^{(f)}}^{p}(I)\right\| \leq 1 .
$$

Using relation (5.1) and the fact that $\widehat{\Psi}_{*}: \mathcal{S}_{f} \rightarrow \mathcal{S}_{g}$ is a unital completely contractive linear map, we deduce that

$$
\left\|\sum_{p=1}^{s}(-1)^{p+1}\left(\begin{array}{l}
s \\
p
\end{array}\right) \Phi_{f, r \widetilde{\varphi}}^{p}(I)\right\| \leq 1, \quad s=1, \ldots, m .
$$

Hence $\sum_{p=1}^{s}(-1)^{p+1}\left(\begin{array}{c}s \\ p\end{array}\right) \Phi_{f, r \widetilde{\varphi}}^{p}(I) \leq I$ and, consequently,

$$
\left(i d-\Phi_{f, r \widetilde{\varphi}}\right)^{s}(I) \geq 0, \quad s=1, \ldots, m,
$$

which shows that $r \widetilde{\varphi}:=\left(r \widetilde{\varphi}_{1}, \ldots, r \widetilde{\varphi}_{n}\right) \in \mathbf{D}_{f}^{m}\left(F^{2}\left(H_{n}\right)\right)$ for any $r \in[0,1)$. Since $\mathbf{D}_{f}^{m}\left(F^{2}\left(H_{n}\right)\right)$ is closed in the operator norm topology, we deduce that $\widetilde{\varphi}:=\left(\widetilde{\varphi}_{1}, \ldots, \widetilde{\varphi}_{n}\right)$ is in the noncommutative domain $\mathbf{D}_{f}^{m}\left(F^{2}\left(H_{n}\right)\right)$.

Setting

$$
\varphi_{i}(X):=\mathbf{B}_{X}\left[\widetilde{\varphi}_{i}\right], \quad X \in \mathbf{D}_{g, \text { rad }}^{l}(\mathcal{H}),
$$

and due to Theorem 2.2 we deduce that the map $\varphi(X):=\left(\varphi_{1}(X), \ldots, \varphi_{n}(X)\right)$ is a free holomorphic function on $\mathbf{D}_{g \text {,rad }}^{l}(\mathcal{H})$ with continuous extension to $\mathbf{D}_{g}^{l}(\mathcal{H})$. This extension is also denoted by $\varphi$. On 
the other hand, since, for each $s=1, \ldots, m,\left(i d-\Phi_{f, r \tilde{\varphi}}\right)^{s}(I)$ is a positive operator in $\mathcal{S}_{g}$ and the noncommutative Berezin transform $\mathcal{B}_{X}$ on the operator system $\mathcal{S}_{g}$ is positive, we deduce that

$$
\left(i d-\Phi_{f, r \varphi(X)}\right)^{s}(I)=\mathcal{B}_{X}\left[\left(i d-\Phi_{f, r \widetilde{\varphi}}\right)^{s}(I)\right] \geq 0
$$

for all $s=1, \ldots, m$. Consequently, $r \varphi(X) \in \mathbf{D}_{f}^{m}(\mathcal{H})$ for $r \in[0,1)$. Since $\mathbf{D}_{f}^{m}(\mathcal{H})$ is closed in the operator norm topology, we deduce that $\varphi(X) \in \mathbf{D}_{f}^{m}(\mathcal{H})$. Therefore, $\varphi$ takes values in $\mathbf{D}_{f}^{m}(\mathcal{H})$. Note also that due to the properties of the noncommutative Berezin transform $\mathcal{B}_{X}$ on the operator system $\mathcal{S}_{g}$ (see relation (1.6)), we have

$$
\varphi_{i}(X)=\mathcal{B}_{X}\left[\widetilde{\varphi}_{i}\right], \quad X \in \mathbf{D}_{g}^{l}(\mathcal{H})
$$

Similarly, we set

$$
\widetilde{\xi}_{i}:=\widehat{\Psi}^{-1}\left(W_{i}^{(g)}\right) \in \mathcal{A}_{n}\left(\mathbf{D}_{f}^{m}\right), \quad i=1, \ldots, q,
$$

and deduce that $\widetilde{\xi}:=\left(\widetilde{\xi}_{1}, \ldots, \widetilde{\xi}_{q}\right)$ is in $\mathbf{D}_{g}^{l}\left(F^{2}\left(H_{n}\right)\right)$. Now, if we define

$$
\xi_{i}(X):=\mathbf{B}_{X}\left[\widetilde{\xi}_{i}\right], \quad X \in \mathbf{D}_{f, \operatorname{rad}}^{m}(\mathcal{H}), i=1, \ldots, q,
$$

then, using again Theorem 2.2, we obtain that the map $\xi(X):=\left(\xi_{1}(X), \ldots, \xi_{q}(X)\right)$ is a free holomorphic function on $\mathbf{D}_{f, \text { rad }}^{m}(\mathcal{H})$ with values in $\mathbf{D}_{g}^{l}(\mathcal{H})$ and continuous extension to $\mathbf{D}_{f}^{m}(\mathcal{H})$, which we also denote by $\xi$. Note that

$$
\xi_{i}(X)=\mathcal{B}_{X}\left[\widetilde{\xi}_{i}\right], \quad X \in \mathbf{D}_{f}^{m}(\mathcal{H}),
$$

where $\mathcal{B}_{X}$ is the noncommutative Berezin transform on the operator system $\mathcal{S}_{f}$. Now, due to Theorem 2.2 , each $\widetilde{\xi}_{i} \in \mathcal{A}_{n}\left(\mathbf{D}_{f}^{m}\right), i=1, \ldots, q$, has a unique Fourier representation $\sum_{\alpha \in \mathbb{F}_{n}^{+}} c_{\alpha} W_{\alpha}^{(f)}$ such that

$$
\widetilde{\xi}_{i}=\lim _{r \rightarrow 1} \sum_{k=0}^{\infty} \sum_{|\alpha|=k} c_{\alpha} r^{|\alpha|} W_{\alpha}^{(f)},
$$

where the limit is in the operator norm topology. Hence, using the continuity of $\widehat{\Psi}$ in the operator norm, and relations (5.4) and (5.1), we obtain

$$
\begin{aligned}
W_{i}^{(g)} & =\widehat{\Psi}\left(\widetilde{\xi}_{i}\right)=\widehat{\Psi}\left(\lim _{r \rightarrow 1} \sum_{k=0}^{\infty} \sum_{|\alpha|=k} c_{\alpha} r^{|\alpha|} W_{\alpha}^{(f)}\right) \\
& =\lim _{r \rightarrow 1} \sum_{k=0}^{\infty} \sum_{|\alpha|=k} c_{\alpha} r^{|\alpha|} \widehat{\Psi}\left(W_{\alpha}^{(f)}\right)=\lim _{r \rightarrow 1} \sum_{k=0}^{\infty} \sum_{|\alpha|=k} c_{\alpha} r^{|\alpha|} \widetilde{\varphi}_{\alpha} .
\end{aligned}
$$

Consequently, using the continuity in the operator norm of the noncommutative Berezin transform at $X \in \mathbf{D}_{g}^{l}(\mathcal{H})$ on the operator system $\mathcal{S}_{g}$, and relations (5.3) and (5.5), we have

$$
\begin{aligned}
X_{i} & =\mathcal{B}_{X}\left[W_{i}^{(g)}\right]=\mathcal{B}_{X}\left[\lim _{r \rightarrow 1} \sum_{k=0}^{\infty} \sum_{|\alpha|=k} c_{\alpha} r^{|\alpha|} \widetilde{\varphi}_{\alpha}\right] \\
& =\lim _{r \rightarrow 1} \sum_{k=0}^{\infty} \sum_{|\alpha|=k} c_{\alpha} r^{|\alpha|} \mathcal{B}_{X}\left[\widetilde{\varphi}_{\alpha}\right]=\lim _{r \rightarrow 1} \sum_{k=0}^{\infty} \sum_{|\alpha|=k} c_{\alpha} r^{|\alpha|} \varphi_{\alpha}(X) \\
& =\lim _{r \rightarrow 1} \mathcal{B}_{\varphi(X)}\left[\sum_{k=0}^{\infty} \sum_{|\alpha|=k} c_{\alpha} r^{|\alpha|} W_{\alpha}^{(f)}\right] \\
& =\mathcal{B}_{\varphi(X)}\left[\widetilde{\xi}_{i}\right]=\xi_{i}(\varphi(X))
\end{aligned}
$$

for each $i=1, \ldots, q$, and any $X \in \mathbf{D}_{g}^{l}(\mathcal{H})$. Hence $(\xi \circ \varphi)(X)=X$ for all $X \in \mathbf{D}_{g}^{l}(\mathcal{H})$. Similarly, one can prove that $(\varphi \circ \xi)(X)=X$ for $X \in \mathbf{D}_{f}^{m}(\mathcal{H})$. Therefore, $\varphi: \mathbf{D}_{g}^{l}(\mathcal{H}) \rightarrow \mathbf{D}_{f}^{m}(\mathcal{H})$ is a homeomorphism such that $\varphi$ and $\varphi^{-1}:=\xi$ are free holomorphic functions on the noncommutative domains $\mathbf{D}_{g, \text { rad }}^{l}(\mathcal{H})$ and $\mathbf{D}_{f, \text { rad }}^{m}(\mathcal{H})$, respectively. This shows that $\varphi \in B i h\left(\mathbf{D}_{g}^{l}, \mathbf{D}_{f}^{m}\right)$. 
Now let $\tilde{\chi} \in \mathcal{A}_{n}\left(\mathbf{D}_{f}^{m}\right)$ have the Fourier representation $\sum_{\alpha \in \mathbb{F}_{n}^{+}} d_{\alpha} W_{\alpha}^{(f)}$. Then

$$
\tilde{\chi}=\lim _{r \rightarrow 1} \sum_{k=0}^{\infty} \sum_{|\alpha|=k} d_{\alpha} r^{|\alpha|} W_{\alpha}^{(f)},
$$

where the limit is in the operator norm topology. According to Theorem 2.2, the map

$$
\chi(X):=\mathcal{B}_{X}[\widetilde{\chi}], \quad X \in \mathbf{D}_{f}^{m}(\mathcal{H})
$$

is in the domain algebra $A\left(\mathbf{D}_{f, \text { rad }}^{m}\right)$. Due to the continuity of the homomorphism $\widehat{\Psi}$ in the operator norm and using relation (5.1), we have

$$
\widehat{\Psi}(\widetilde{\chi})=\lim _{r \rightarrow 1} \sum_{k=0}^{\infty} \sum_{|\alpha|=k} d_{\alpha} r^{|\alpha|} \widetilde{\varphi}_{\alpha} .
$$

Hence, using relations (5.3) $(\underline{5.6})$, and applying the noncommutative Berezin transform at $X \in \mathbf{D}_{g}^{l}(\mathcal{H})$ on the operator system $\mathcal{S}_{g}$ and using its continuity in norm, we get

$$
\begin{aligned}
{[\Psi(\chi)](X) } & =\mathcal{B}_{X}[\widehat{\Psi}(\widetilde{\chi})]=\lim _{r \rightarrow 1} \sum_{k=0}^{\infty} \sum_{|\alpha|=k} d_{\alpha} r^{|\alpha|} \mathcal{B}_{X}\left[\widetilde{\varphi}_{\alpha}\right] \\
& =\lim _{r \rightarrow 1} \sum_{k=0}^{\infty} \sum_{|\alpha|=k} d_{\alpha} r^{|\alpha|} \varphi_{\alpha}(X) \\
& =\lim _{r \rightarrow 1} \mathcal{B}_{\varphi(X)}\left[\sum_{k=0}^{\infty} \sum_{|\alpha|=k} d_{\alpha} r^{|\alpha|} W_{\alpha}^{(f)}\right] \\
& =\mathcal{B}_{\varphi(X)}[\widetilde{\chi}]=(\chi \circ \varphi)(X)
\end{aligned}
$$

for any $X \in \mathbf{D}_{g}^{l}(\mathcal{H})$, which completes the proof of the implication $(i) \Longrightarrow(i i)$.

To prove that $(i i) \Longrightarrow(i)$, assume that $\varphi \in B i h\left(\mathbf{D}_{g}^{l}, \mathbf{D}_{f}^{m}\right)$ and define $\Psi(\chi):=\chi \circ \varphi$ for $\chi \in A\left(\mathbf{D}_{f, \text { rad }}^{m}\right)$. First note that, due to Theorem 3.3. $\chi \circ \varphi \in A\left(\mathbf{D}_{g \text {,rad }}^{l}\right)$ for all $\chi \in A\left(\mathbf{D}_{f, \text { rad }}^{m}\right)$, and $\Psi$ is a well-defined completely contractive homomorphism. A similar result can be deduced for the map $\Lambda(\chi):=\chi \circ \varphi^{-1}$, $\chi \in A\left(\mathbf{D}_{g, \text { rad }}^{l}\right)$. Now, note that, for any $\chi \in A\left(\mathbf{D}_{f, \text { rad }}^{m}\right)$ and $X \in \mathbf{D}_{f}^{m}(\mathcal{H})$, we have $(\Lambda \circ \Psi)(\chi)=(\chi \circ \varphi) \circ \varphi^{-1}$ and

$$
\begin{aligned}
{\left[(\chi \circ \varphi) \circ \varphi^{-1}\right](X) } & =(\chi \circ \varphi)\left(\varphi^{-1}(X)\right)=\lim _{r \rightarrow 1} \chi\left(r \varphi_{1}\left(\varphi^{-1}(X)\right), \ldots, r \varphi_{n}\left(\varphi^{-1}(X)\right)\right) \\
& =\lim _{r \rightarrow 1} \chi\left(r X_{1}, \ldots, r X_{n}\right)=\chi\left(X_{1}, \ldots, X_{n}\right),
\end{aligned}
$$

where the convergence is in the operator norm topology. Hence, $\Lambda \circ \Psi=i d$. Similarly, we can show that $\Psi \circ \Lambda=i d$. Since $\Psi$ and $\Lambda$ are completely contractive homomorphisms, we deduce that $\Psi$ is a completely isometric isomorphism. Moreover, since $\varphi:=\left(\varphi_{1}, \ldots, \varphi_{n}\right) \in \operatorname{Bih}\left(\mathbf{D}_{g}^{l}\right.$, $\left.\mathbf{D}_{f}^{m}\right)$, we can use Theorem 2.2 and deduce that $\widetilde{\varphi}:=\left(\widetilde{\varphi}_{1}, \ldots, \widetilde{\varphi}_{n}\right) \in \mathbf{D}_{f}^{m}\left(F^{2}\left(H_{n}\right)\right)$ and $\widetilde{\varphi}_{i} \in \mathcal{A}_{q}\left(\mathbf{D}_{g}^{l}\right)$. Consequently, using the noncommutative von Neumann inequality (1.7), we deduce that the linear map

$$
\mathcal{S}_{f} \ni W_{\alpha}^{(f)} W_{\beta}^{(f)^{*}} \mapsto \widetilde{\varphi}_{\alpha} \widetilde{\varphi}_{\beta}^{*} \in \mathcal{S}_{g}, \quad \alpha, \beta \in \mathbb{F}_{n}^{+},
$$

is completely contractive. A similar result can be deduced for $\varphi^{-1}$. Therefore, $\Psi$ has completely contractive hereditary extension, and item (i) holds. Moreover, due to Theorem 3.3 , we have $\widehat{\Psi}(\tilde{\chi})=\mathcal{B}_{\tilde{\varphi}}[\tilde{\chi}]$, $\tilde{\chi} \in \mathcal{A}_{n}\left(\mathbf{D}_{f}^{m}\right)$, where $\mathcal{B}_{\widetilde{\varphi}}$ is the noncommutative Berezin transform at $\widetilde{\varphi}$.

Now, we consider the particular case when $m=l=1$. Let $\widehat{\Psi}: \mathcal{A}_{n}\left(\mathbf{D}_{f}^{1}\right) \rightarrow \mathcal{A}_{q}\left(\mathbf{D}_{g}^{1}\right)$ be a completely isometric isomorphism. Then, we showed at the beginning of this proof that $\widetilde{\varphi}:=\left(\widetilde{\varphi}_{1}, \ldots, \widetilde{\varphi}_{n}\right) \in$ $\mathbf{D}_{f}^{1}\left(F^{2}\left(H_{n}\right)\right)$, where $\widetilde{\varphi}_{i}$ are given by relation (5.1). Similarly, we deduce that $\widetilde{\xi}:=\left(\widetilde{\xi}_{1}, \ldots, \widetilde{\xi}_{q}\right)$ is in $\mathbf{D}_{g}^{1}\left(F^{2}\left(H_{n}\right)\right.$ ), where $\widetilde{\xi}_{i}$ are given by relation (5.4). As above, using the noncommutative von Neumann inequality (1.7), we deduce that $\Psi$ has unital completely contractive hereditary extension, which completes the proof. 
Corollary 5.2. Let $f$ and $g$ be positive regular free holomorphic functions with $n$ and $q$ indeterminates, respectively. Then the noncommutative domains $\mathbf{D}_{f}^{1}(\mathcal{H})$ and $\mathbf{D}_{g}^{1}(\mathcal{H})$ are free biholomorphic equivalent if and only if the domain algebras $\mathcal{A}_{n}\left(\mathbf{D}_{f}^{1}\right)$ and $\mathcal{A}_{q}\left(\mathbf{D}_{g}^{1}\right)$ are completely isometrically isomorphic.

Let us consider an example. In 2], Arias and Latrémolière showed that if $\omega:=X_{1}+X_{2}+X_{1} X_{2}$ and $\xi:=X_{1}+X_{2}+\frac{1}{2}\left(X_{1} X_{2}+X_{2} X_{1}\right)$, then the noncommutative domain algebras $\mathcal{A}_{2}\left(\mathbf{D}_{\omega}^{1}\right)$ and $\mathcal{A}_{2}\left(\mathbf{D}_{\xi}^{1}\right)$ are not completely isometrically isomorphic. Therefore, according to Corollary 5.2 the noncommutative domains $\mathbf{D}_{\omega}^{1}(\mathcal{H})$ and $\mathbf{D}_{\xi}^{1}(\mathcal{H})$ are not free biholomorphic equivalent.

Now, using Theorem 4.3, Corollary 5.2, and Arias-Latrémolière characterization of the noncommutative disc algebra $\mathcal{A}_{n}$ (see Theorem 4.7 from [2]), we deduce the following classification result.

Corollary 5.3. Let $g$ be a positive regular free holomorphic function with $q$ indeterminates. Then the noncommutative domain $\mathbf{D}_{g}^{1}(\mathcal{H})$ is biholomorphic equivalent to the unit ball $\left[B(\mathcal{H})^{n}\right]_{1}$ if and only if $q=n$ and $g=c_{1} X_{1}+\cdots+c_{n} X_{n}$ for some $c_{i}>0$.

Proof. Let $p:=X_{1}+\cdots+X_{n}$ and recall that $\mathbf{D}_{p}^{1}(\mathcal{H})=\left[B(\mathcal{H})^{n}\right]_{1}$. Due to Theorem 4.3 and Corollary 5.2. $\mathbf{D}_{g}^{1}(\mathcal{H})$ is free biholomorphic equivalent to the unit ball $\left[B(\mathcal{H})^{n}\right]_{1}$ if and only if $q=n$ and the domain algebras $\mathcal{A}_{n}$ and $\mathcal{A}_{n}\left(\mathbf{D}_{g}^{l}\right)$ are completely isometrically isomorphic. Applying Arias-Latrémolière result, the result follows. The converse is obvious by rescaling.

Corollary 5.4. Let $f$ and $g$ be positive regular free holomorphic functions with $n$ and $q$ indeterminates, respectively. Let $\Psi: A\left(\mathbf{D}_{f, \text { rad }}^{m}\right) \rightarrow A\left(\mathbf{D}_{g, \text { rad }}^{l}\right)$ be a unital algebra homomorphism. Then $\Psi$ is a unital completely isometric isomorphism having completely contractive hereditary extension if and only if $\Psi$ is a continuous homeomorphism such that the $n$-tuples of operators $\left(\hat{\Psi}\left(W_{1}^{(f)}\right), \ldots, \hat{\Psi}\left(W_{n}^{(f)}\right)\right)$ and $\left(\hat{\Psi}^{-1}\left(W_{1}^{(g)}\right), \ldots, \hat{\Psi}^{-1}\left(W_{q}^{(g)}\right)\right)$ are in $\mathbf{D}_{f}^{m}\left(F^{2}\left(H_{n}\right)\right)$ and $\mathbf{D}_{g}^{l}\left(F^{2}\left(H_{q}\right)\right)$, respectively.

Proof. The direct implication follows from the proof of Theorem [5.1. Conversely, assume that $\Psi$ is a continuous homeomorphism such that $\left(\hat{\Psi}\left(W_{1}^{(f)}\right), \ldots, \hat{\Psi}\left(W_{n}^{(f)}\right)\right)$ is in $\mathbf{D}_{f}^{m}\left(F^{2}\left(H_{n}\right)\right)$. Due to the noncommutative von Neumann inequality (see (1.7)), we have

$$
\left\|\left[\hat{\Psi}\left(p_{i j}\left(W_{1}^{(f)}, \ldots, W_{n}^{(f)}\right)\right)\right]_{k \times k}\right\|=\|\left[p_{i j}\left(\hat{\Psi}\left(W_{1}^{(f)}\right), \ldots, \hat{\Psi}\left(W_{n}^{(f)}\right)\right]_{k \times k}\|\leq\|\left[p_{i j}\left(W_{1}^{(f)}, \ldots, W_{n}^{(f)}\right)\right]_{k \times k} \|\right.
$$

for any operator matrix $\left[p_{i j}\left(W_{1}^{(f)}, \ldots, W_{n}^{(f)}\right)\right]_{k \times k} \in \mathcal{A}_{n}\left(\mathbf{D}_{f}^{m}\right) \otimes M_{k \times k}$ and $k \geq 1$. Since $\hat{\Psi}$ is continuous on $\mathcal{A}_{n}\left(\mathbf{D}_{f}^{m}\right)$, which is the norm closed algebra generated by $W_{1}^{(f)}, \ldots, W_{n}^{(f)}$ and the identity, we deduce that $\hat{\Psi}: \mathcal{A}_{n}\left(\mathbf{D}_{f}^{m}\right) \rightarrow \mathcal{A}_{q}\left(\mathbf{D}_{g}^{l}\right)$ is a unital completely contractive homomorphism. Similarly, if $\left(\hat{\Psi}^{-1}\left(W_{1}^{(g)}\right), \ldots, \hat{\Psi}^{-1}\left(W_{q}^{(g)}\right)\right)$ is in $\mathbf{D}_{g}^{l}\left(F^{2}\left(H_{q}\right)\right)$, we can prove that $\hat{\Psi}^{-1}$ is a unital completely contractive homomorphism. Therefore, $\hat{\Psi}$ is a complete isometry. Moreover, using the noncommutative von Neumann inequality (1.7), we deduce that $\Psi$ has completely contractive hereditary extension, and complete the proof.

We denote by $\operatorname{Aut}\left(\mathbf{D}_{f}^{m}\right):=\operatorname{Bih}\left(\mathbf{D}_{f}^{m}, \mathbf{D}_{f}^{m}\right)$ the automorphism group of $\mathbf{D}_{f}^{m}(\mathcal{H})$. The group of all unital completely isometric automorphisms of the noncommutative domain algebra $A\left(\mathbf{D}_{f, \text { rad }}^{m}\right)$ is denoted by $A u t_{c i}\left(A\left(\mathbf{D}_{f, \text { rad }}^{m}\right)\right)$. We also use the notation $A u t_{c i}^{*}\left(A\left(\mathbf{D}_{f, \text { rad }}^{m}\right)\right)$ for the set of all automorphisms $\Psi \in$ $A u t_{c i}\left(A\left(\mathbf{D}_{f, \text { rad }}^{m}\right)\right)$ having completely contractive hereditary extension.

Corollary 5.5. Let $f$ be a positive regular free holomorphic function with $n$ indeterminates, and let $m \geq 1$. Then the following statements are equivalent:

(i) $\Psi \in A u t_{c i}^{*}\left(A\left(\mathbf{D}_{f, \text { rad }}^{m}\right)\right)$;

(ii) there is $\varphi \in \operatorname{Aut}\left(\mathbf{D}_{f}^{m}\right)$ such that

$$
\Psi(\chi)=\chi \circ \varphi, \quad \chi \in A\left(\mathbf{D}_{f, \mathrm{rad}}^{m}\right) .
$$


Consequently, Aut ${ }_{c i}^{*}\left(A\left(\mathbf{D}_{f, \mathrm{rad}}^{m}\right)\right)$ is a subgroup of $A u t_{c i}\left(A\left(\mathbf{D}_{f, \mathrm{rad}}^{m}\right)\right)$ and

$$
A u t_{c i}^{*}\left(A\left(\mathbf{D}_{f, \mathrm{rad}}^{m}\right)\right) \simeq \operatorname{Aut}\left(\mathbf{D}_{f}^{m}\right) .
$$

In the particular case when $m=1$,

$$
A u t_{c i}^{*}\left(A\left(\mathbf{D}_{f, \mathrm{rad}}^{1}\right)\right)=A u t_{c i}\left(A\left(\mathbf{D}_{f, \mathrm{rad}}^{1}\right)\right) \simeq \operatorname{Aut}\left(\mathbf{D}_{f}^{1}\right) .
$$

We mention that in the particular case when $m=1$ and $f=X_{1}+\cdots+X_{n}$, Corollary [5.5] was obtained in [30] using different methods. In addition, we used the theory of characteristic functions for row contractions (see [18]) to determine the group $A u t\left[B(\mathcal{H})^{n}\right]_{1}$ ) of all free holomorphic automorphisms of $\left[B(\mathcal{H})^{n}\right]_{1}$. It was shown that $A u t\left(\left[B(\mathcal{H})^{n}\right]_{1}\right) \simeq A u t\left(\mathbb{B}_{n}\right)$, the Moebius group of the open unit ball $\mathbb{B}_{n}:=\left\{\lambda \in \mathbb{C}^{n}:\|\lambda\|_{2}<1\right\}$.

If $\Psi: A\left(\mathbf{D}_{f, \mathrm{rad}}^{m}\right) \rightarrow A\left(\mathbf{D}_{g, \mathrm{rad}}^{l}\right)$ is a unital completely isometric isomorphism having completely contractive hereditary extension, then due to Theorem [5.1] $\Psi(\chi)=\chi \circ \varphi, \chi \in A\left(\mathbf{D}_{f \text {,rad }}^{m}\right)$, for some $\varphi \in \operatorname{Bih}\left(\mathbf{D}_{g}^{l}, \mathbf{D}_{f}^{m}\right)$. In this case, we call $\varphi$ the symbol of $\Psi$. Using Theorem 4.5 and Theorem 5.1 , we can deduce the following result.

Theorem 5.6. Let $f$ and $g$ be positive regular free holomorphic functions with $n$ and $q$ indeterminates, respectively. A map $\Psi: A\left(\mathbf{D}_{f, \mathrm{rad}}^{m}\right) \rightarrow A\left(\mathbf{D}_{g, \mathrm{rad}}^{l}\right)$ is a unital completely isometric isomorphism having completely contractive hereditary extension and such that its symbol $\varphi$ fixes the origin if and only if $n=q$ and $\Psi$ is given by

$$
\Psi(\chi)=\chi \circ \varphi, \quad \chi \in A\left(\mathbf{D}_{f, \text { rad }}^{m}\right)
$$

for some $\varphi \in \operatorname{Bih}\left(\mathbf{D}_{g}^{l}, \mathbf{D}_{f}^{m}\right)$ of the form

$$
\varphi\left(X_{1}, \ldots X_{n}\right)=\left[X_{1}, \ldots, X_{n}\right] U, \quad\left(X_{1}, \ldots, X_{n}\right) \in \mathbf{D}_{g, \text { rad }}^{l}(\mathcal{H}),
$$

where $U$ is an invertible operator on $\mathbb{C}^{n}$ such that

$$
\left[W_{1}^{(g)}, \ldots, W_{n}^{(g)}\right] U \in \mathbf{D}_{f}^{m}\left(F^{2}\left(H_{n}\right)\right) \quad \text { and } \quad\left[W_{1}^{(f)}, \ldots, W_{n}^{(f)}\right] U^{-1} \in \mathbf{D}_{g}^{l}\left(F^{2}\left(H_{n}\right)\right) .
$$

In this case, we have

$$
\left[\widehat{\Psi}\left(W_{1}^{(f)}\right), \ldots, \widehat{\Psi}\left(W_{n}^{(f)}\right)\right]=\widetilde{\varphi}=\left[W_{1}^{(g)}, \ldots, W_{n}^{(g)}\right] U .
$$

We mention that, in the particular case when $m=l=1$, Arias and Latrémolière proved (see Theorem 3.18 from [2]) that if there is an completely isometric isomorphism between two noncommutative domain algebras $\mathcal{A}_{n}\left(\mathbf{D}_{f}^{1}\right)$ and $\mathcal{A}_{n}\left(\mathbf{D}_{g}^{1}\right)$ whose dual map fixes the origin, then the algebras are related by a linear relation of their generators. Our Theorem 5.6 implies and strengthens their result and also provides a converse.

\section{ISOMORPHISMS OF NONCOMMUTATIVE HARDY ALGEBRAS}

In this section we characterize the unitarily implemented isomorphisms of noncommutative Hardy algebras associated with noncommutative domains. Similar results are deduced for noncommutative domain algebras.

Let $f$ and $g$ be positive regular free holomorphic functions with $n$ and $q$ indeterminates, respectively, and let $m, l \geq 1$. According to Theorem 2.1, there is a unital completely isometric isomorphism

$$
H^{\infty}\left(\mathbf{D}_{f, \mathrm{rad}}^{m}\right) \ni \chi \mapsto \tilde{\chi}:=\mathrm{SOT}-\lim _{r \rightarrow 1} \chi\left(r W_{1}, \ldots, r W_{n}\right) \in F_{n}^{\infty}\left(\mathbf{D}_{f}^{m}\right),
$$

where the limit is in the strong operator topology, whose inverse is the noncommutative Berezin transform B, i.e., $\chi(X)=\mathbf{B}_{X}[\widetilde{\chi}]$ for $X \in \mathbf{D}_{f, \text { rad }}^{m}(\mathcal{H})$. If $\Psi: H^{\infty}\left(\mathbf{D}_{f, \text { rad }}^{m}\right) \rightarrow H^{\infty}\left(\mathbf{D}_{g, \text { rad }}^{l}\right)$ is a unital homomorphism, it induces a unique unital homomorphism $\widehat{\Psi}: F_{n}^{\infty}\left(\mathbf{D}_{f}^{m}\right) \rightarrow F_{q}^{\infty}\left(\mathbf{D}_{g}^{l}\right)$ such that the diagram

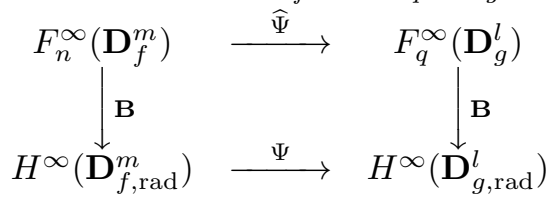


is commutative, i.e., $\Psi \mathbf{B}=\mathbf{B} \widehat{\Psi}$, where $\mathbf{B}$ is the appropriate noncommutative Berezin transform on the Hardy space $F_{n}^{\infty}\left(\mathbf{D}_{f}^{m}\right)$ or $F_{q}^{\infty}\left(\mathbf{D}_{g}^{l}\right)$. The homomorphisms $\Psi$ and $\widehat{\Psi}$ uniquely determine each other by the formulas:

$$
\begin{aligned}
{[\Psi(\chi)](X) } & =\mathbf{B}_{X}[\widehat{\Psi}(\tilde{\chi})], \quad \chi \in H^{\infty}\left(\mathbf{D}_{f, \mathrm{rad}}^{m}\right), \quad X \in \mathbf{D}_{g, \mathrm{rad}}^{l}(\mathcal{H}), \quad \text { and } \\
\widehat{\Psi}(\tilde{\chi}) & =\widetilde{\Psi(\chi)}, \quad \tilde{\chi} \in F_{n}^{\infty}\left(\mathbf{D}_{f}^{m}\right) .
\end{aligned}
$$

If $\widehat{\Psi}$ is is a SOT-continuous homomorphism, we say that $\Psi$ has the same property.

A bijective map $F: \mathbf{D}_{f, \text { rad }}^{m}(\mathcal{H}) \rightarrow \mathbf{D}_{g \text {,rad }}^{l}(\mathcal{H})$ is called free biholomorphic function if $F$ and $F^{-1}$ are free holomorphic functions on $\mathbf{D}_{f, \text { rad }}^{m}(\mathcal{H})$ and $\mathbf{D}_{g, \text { rad }}^{l}(\mathcal{H})$, respectively. We denote by $\operatorname{Bih}\left(\mathbf{D}_{g, \text { rad }}^{l}, \mathbf{D}_{f, \text { rad }}^{m}\right)$ the set of all free biholomorphic functions from $\mathbf{D}_{g \text {,rad }}^{l}(\mathcal{H})$ onto $\mathbf{D}_{f, \text { rad }}^{m}(\mathcal{H})$. Note the difference between $\operatorname{Bih}\left(\mathbf{D}_{g, \text { rad }}^{l}, \mathbf{D}_{f, \text { rad }}^{m}\right)$ and $\operatorname{Bih}\left(\mathbf{D}_{g}^{l}, \mathbf{D}_{f}^{m}\right)$.

Theorem 6.1. Let $f$ and $g$ be positive regular free holomorphic functions with $n$ and $q$ indeterminates, respectively. If $\psi \in \operatorname{Bih}\left(\mathbf{D}_{g, \mathrm{rad}}^{l}, \mathbf{D}_{f, \mathrm{rad}}^{m}\right)$, then the map

$$
\Psi(\chi):=\chi \circ \psi, \quad \chi \in H^{\infty}\left(\mathbf{D}_{f, \text { rad }}^{m}\right),
$$

is a completely isometric isomorphism from the Hardy algebra $H^{\infty}\left(\mathbf{D}_{f, \mathrm{rad}}^{m}\right)$ onto $H^{\infty}\left(\mathbf{D}_{g, \mathrm{rad}}^{l}\right)$. If, in addition, the model boundary function $\tilde{\psi}$ is a pure $n$-tuple, then $\Psi$ is sequentially SOT-continuous.

In particular, if $\psi \in \operatorname{Bih}\left(\mathbf{D}_{g}^{l}, \mathbf{D}_{f}^{m}\right)$ and $\psi(0)=0$, then $\Psi$ is a completely isometric isomorphism and has the form mentioned in Theorem 5.6.

Proof. Using Theorem [3.2, we deduce that $\chi \circ \psi \in H^{\infty}\left(\mathbf{D}_{g, \text { rad }}^{l}\right)$ and $\|\chi \circ \psi\|_{\infty} \leq\|\chi\|_{\infty}$. Moreover, passing to matrices, we can similarly show that $\Psi$ is a completely contractive homomorphism. In the same manner, one can prove that the map $\Gamma(\chi):=\chi \circ \psi^{-1}$ is a completely contractive homomorphism from $H^{\infty}\left(\mathbf{D}_{g, \text { rad }}^{l}\right)$ to $H^{\infty}\left(\mathbf{D}_{f, \text { rad }}^{m}\right)$. Let $\chi \in H^{\infty}\left(\mathbf{D}_{f, \text { rad }}^{m}\right)$ have the representation $\sum_{k=0}^{\infty} \sum_{|\alpha|=k} c_{\alpha} Z_{\alpha}$, and fix $X:=\left(X_{1}, \ldots, X_{n}\right) \in \mathbf{D}_{f \text {,rad }}^{m}(\mathcal{H})$. Since $\psi^{-1}(X) \in \mathbf{D}_{g \text {,rad }}^{m}(\mathcal{H})$ and $\psi\left(\mathbf{D}_{g \text {,rad }}^{l}(\mathcal{H})\right) \subset \mathbf{D}_{f, \text { rad }}^{m}(\mathcal{H})$ we deduce that

$$
(\chi \circ \psi)\left(\psi^{-1}(X)\right)=\sum_{k=0}^{\infty} \sum_{|\alpha|=k} c_{\alpha} \psi_{\alpha}\left(\psi^{-1}(X)\right),
$$

where the convergence is in the operator norm topology. Since $\psi \in \operatorname{Bih}\left(\mathbf{D}_{g, \text { rad }}^{l}, \mathbf{D}_{f, \text { rad }}^{m}\right)$, we have $\psi_{i}\left(\psi^{-1}(X)\right)=X_{i}, i=1, \ldots, n$, and, therefore, $\psi_{\alpha}\left(\psi^{-1}(X)\right)=X_{\alpha}$ for $\alpha \in \mathbb{F}_{n}^{+}$. Consequently, $(\Gamma \circ \Psi)(\chi)=$ $\chi$ for any $\chi \in H^{\infty}\left(\mathbf{D}_{f, \text { rad }}^{m}\right)$. Similarly, one can prove that $\Phi \circ \Gamma=i d$. Summing up, we conclude that the map $\Psi$ is a completely isometric isomorphism of noncommutative Hardy algebras.

If, in addition, we assume that $\tilde{\psi}$ is a pure $n$-tuple, then, according to Theorem 3.5, we have

$$
\widehat{\Psi}(\widetilde{\chi})=\widetilde{\Psi(\chi)}=\widetilde{\chi \circ \psi}=\mathcal{B}_{\widetilde{\psi}}[\widetilde{\chi}]=K_{\widetilde{\psi}, f}^{(m)^{*}}\left[\widetilde{\chi} \otimes I_{F^{2}\left(H_{n}\right)}\right] K_{\widetilde{\psi}, f}^{(m)},
$$

where $\mathcal{B}_{\widetilde{\psi}}$ is the noncommutative Berezin transform at the pure $n$-tuple $\widetilde{\psi}$ (see relation (2.1)). Consequently, $\Psi$ is SOT-continuous on bounded sets.

Now, we prove the last part of the theorem. According to Theorem 4.5, if $\psi \in \operatorname{Bih}\left(\mathbf{D}_{g}^{l}, \mathbf{D}_{f}^{m},\right)$ and $\psi(0)=0$, then $n=q$ and $\psi$ has the form

$$
\psi\left(X_{1}, \ldots, X_{n}\right)=\left[X_{1}, \ldots, X_{n}\right] U, \quad\left(X_{1}, \ldots, X_{n}\right) \in \mathbf{D}_{g}^{l}(\mathcal{H}),
$$

where $U$ is an invertible operator on $\mathbb{C}^{n}$ such that

$$
\left[W_{1}^{(g)}, \ldots, W_{n}^{(g)}\right] U \in \mathbf{D}_{f}^{m}\left(F^{2}\left(H_{n}\right)\right) \quad \text { and } \quad\left[W_{1}^{(f)}, \ldots, W_{n}^{(f)}\right] U^{-1} \in \mathbf{D}_{g}^{l}\left(F^{2}\left(H_{n}\right)\right) .
$$

Hence, it is obvious that we have $\psi\left(r W_{1}^{(g)}, \ldots, r W_{n}^{(g)}\right)=r \psi\left(W_{1}^{(g)}, \ldots, W_{n}^{(g)}\right) \in \mathbf{D}_{f, \text { rad }}^{m}\left(F^{2}\left(H_{n}\right)\right)$ and $\psi^{-1}\left(r W_{1}^{(f)}, \ldots, r W_{n}^{(f)}\right)=r \psi^{-1}\left(W_{1}^{(f)}, \ldots, W_{n}^{(f)}\right) \in \mathbf{D}_{g, \text { rad }}^{l}\left(F^{2}\left(H_{n}\right)\right)$ for any $r \in[0,1)$. Due to Lemma 3.1. we deduce that $\psi\left(\mathbf{D}_{g, \mathrm{rad}}^{l}\right) \subseteq \mathbf{D}_{f, \mathrm{rad}}^{m}$ and $\psi^{-1}\left(\mathbf{D}_{f, \mathrm{rad}}^{m}\right) \subseteq \mathbf{D}_{g, \mathrm{rad}}^{l}$. Therefore, $\psi \in \operatorname{Bih}\left(\mathbf{D}_{g, \mathrm{rad}}^{l}, \mathbf{D}_{f, \mathrm{rad}}^{m}\right)$. Applying the first part of this theorem, we complete the proof. 
We introduce now the set $\operatorname{Bih}\left(\mathbf{D}_{g, \text { pure }}^{l}, \mathbf{D}_{f, \text { pure }}^{m}\right)$ of all bijections $\varphi: \mathbf{D}_{g, \text { pure }}^{l}(\mathcal{H}) \rightarrow \mathbf{D}_{f, \text { pure }}^{m}(\mathcal{H})$ such that $\left.\varphi\right|_{\mathbf{D}_{g, \text { rad }}^{l}(\mathcal{H})}$ and $\left.\varphi^{-1}\right|_{\mathbf{D}_{f, \text { rad }}^{m}(\mathcal{H})}$ are pure free holomorphic functions, i.e., their model boundary functions are pure, and $\varphi$ and $\varphi^{-1}$ are their radial extensions in the strong operator topology, respectively, i.e.,

$$
\varphi(X)=\text { SOT- } \lim _{r \rightarrow 1} \varphi(r X), \quad X \in \mathbf{D}_{g, \text { pure }}^{l}(\mathcal{H}),
$$

and

$$
\varphi^{-1}(X)=\text { SOT }-\lim _{r \rightarrow 1} \varphi^{-1}(r X), \quad X \in \mathbf{D}_{f, \text { pure }}^{m}(\mathcal{H})
$$

Theorem 6.2. Let $f$ and $g$ be positive regular free holomorphic functions with $n$ and $q$ indeterminates, respectively. A map $\Psi: H^{\infty}\left(\mathbf{D}_{f, \mathrm{rad}}^{m}\right) \rightarrow H^{\infty}\left(\mathbf{D}_{g, \mathrm{rad}}^{l}\right)$ is a unitarily implemented isomorphism if and only if it has the form

$$
\Psi(\chi):=\chi \circ \varphi, \quad \chi \in H^{\infty}\left(\mathbf{D}_{f, \mathrm{rad}}^{m}\right)
$$

for some $\varphi \in \operatorname{Bih}\left(\mathbf{D}_{g, \text { pure }}^{l}, \mathbf{D}_{f, \text { pure }}^{m}\right)$ with the property that $\tilde{\varphi}$ is unitarily equivalent to the universal model $\left(W_{1}^{(f)}, \ldots, W_{n}^{(f)}\right)$ associated with $\mathbf{D}_{f}^{m}$. In this case,

$$
\widehat{\Psi}(\widetilde{\chi})=\mathcal{B}_{\tilde{\varphi}}[\widetilde{\chi}]:=K_{f, \widetilde{\varphi}}^{(m)^{*}}\left(\widetilde{\chi} \otimes I_{\mathcal{D}_{f, m, \tilde{\varphi}}}\right) K_{f, \widetilde{\varphi}}^{(m)}, \quad \tilde{\chi} \in F_{n}^{\infty}\left(\mathbf{D}_{f}^{m}\right),
$$

where the noncommutative Berezin kernel $K_{f, \widetilde{\varphi}}^{(m)}$ is a unitary operator and $\operatorname{dim} \mathcal{D}_{f, m, \widetilde{\varphi}}=1$.

Proof. Assume that the induced map $\widehat{\Psi}: F_{n}^{\infty}\left(\mathbf{D}_{f}^{m}\right) \rightarrow F_{q}^{\infty}\left(\mathbf{D}_{g}^{l}\right)$ is a unitarily implemented isomorphism. Then, there is a unitary operator $U \in B\left(F^{2}\left(H_{q}\right), F^{2}\left(H_{n}\right)\right)$ such that $\widehat{\Psi}(\widetilde{\chi})=U^{*} \tilde{\chi} \in F_{q}^{\infty}\left(\mathbf{D}_{g}^{l}\right)$ for all $\tilde{\chi} \in F_{n}^{\infty}\left(\mathbf{D}_{f}^{m}\right)$. Denote

$$
\widetilde{\varphi}_{i}:=\widehat{\Psi}\left(W_{i}^{(f)}\right) \in F_{q}^{\infty}\left(\mathbf{D}_{g}^{l}\right), \quad i=1, \ldots, n
$$

Since $\widetilde{\varphi}:=\left(\widetilde{\varphi}_{1}, \ldots, \widetilde{\varphi}_{n}\right)$ is unitarily equivalent to $\left(W_{1}^{(f)}, \ldots, W_{n}^{(f)}\right)$, which is a pure $n$-tuple in the noncommutative domain $\mathbf{D}_{f}^{m}\left(F^{2}\left(H_{n}\right)\right)$, so is the $n$-tuple $\widetilde{\varphi}$. Setting

$$
\varphi_{i}(X):=\mathcal{B}_{X}\left[\widetilde{\varphi}_{i}\right], \quad X \in \mathbf{D}_{q, \text { pure }}^{l}(\mathcal{H}),
$$

and due to Theorem 2.1 and Lemma [3.4 the map $\varphi(X):=\left(\varphi_{1}(X), \ldots, \varphi_{n}(X)\right)$ is a bounded free holomorphic function on $\mathbf{D}_{g \text {,rad }}^{l}(\mathcal{H})$ with values in $\mathbf{D}_{f, \text { pure }}^{m}(\mathcal{H})$. Moreover, due to the properties of the noncommutative Berezin transform (see (2.1) $)$, we have $\varphi(X)=\operatorname{SOT}-\lim _{r \rightarrow 1} \varphi(r X)$ for $X \in \mathbf{D}_{g, \text { pure }}^{l}(\mathcal{H})$.

Similarly, we set

$$
\widetilde{\xi}_{i}:=\widehat{\Psi}^{-1}\left(W_{i}^{(g)}\right) \in F_{n}^{\infty}\left(\mathbf{D}_{f}^{m}\right), \quad i=1, \ldots, q
$$

and using the fact that $\widehat{\Psi}^{-1}: F_{q}^{\infty}\left(\mathbf{D}_{g}^{l}\right) \rightarrow F_{n}^{\infty}\left(\mathbf{D}_{f}^{m}\right)$ is given by $\widehat{\Psi}^{-1}(\widetilde{\zeta})=U \widetilde{\zeta} U^{*}, \widetilde{\zeta} \in F_{q}^{\infty}\left(\mathbf{D}_{g}^{l}\right)$, we deduce that $\widetilde{\xi}:=\left(\widetilde{\xi}_{1}, \ldots, \widetilde{\xi}_{q}\right)$ is a pure $q$-tuple in $\mathbf{D}_{g}^{l}\left(F^{2}\left(H_{n}\right)\right)$. Now, if we define

$$
\xi_{i}(X):=\mathcal{B}_{X}\left[\widetilde{\xi}_{i}\right], \quad X \in \mathbf{D}_{f, \text { pure }}^{m}(\mathcal{H}), i=1, \ldots, q,
$$

then, using again Theorem 2.1 and Lemma 3.4, we obtain that the map $\xi(X):=\left(\xi_{1}(X), \ldots, \xi_{n}(X)\right)$ is a bounded free holomorphic function on $\mathbf{D}_{f, \text { rad }}^{m}(\mathcal{H})$ with values in $\mathbf{D}_{g \text {,pure }}^{l}(\mathcal{H})$ and has the property that $\xi(X)=$ SOT- $\lim _{r \rightarrow 1} \xi(r X)$ for $X \in \mathbf{D}_{f, \text { pure }}^{m}(\mathcal{H})$. Now, due to Theorem 2.1] each $\widetilde{\xi}_{i} \in F_{n}^{\infty}\left(\mathbf{D}_{f}^{m}\right)$, $i=1, \ldots, q$, has a unique Fourier representation $\sum_{\alpha \in \mathbb{F}_{n}^{+}} c_{\alpha} W_{\alpha}^{(f)}$ such that

$$
\widetilde{\xi}_{i}=\text { SOT- } \lim _{r \rightarrow 1} \sum_{k=0}^{\infty} \sum_{|\alpha|=k} c_{\alpha} r^{|\alpha|} W_{\alpha}^{(f)}
$$


where the limit is in the strong operator topology. Hence, using the continuity of $\widehat{\Psi}$ in the strong operator topology, and relations (6.1) and (6.3), we obtain

$$
\begin{aligned}
W_{i}^{(g)} & =\widehat{\Psi}\left(\widetilde{\xi}_{i}\right)=\widehat{\Psi}\left(\text { SOT }-\lim _{r \rightarrow 1} \sum_{k=0}^{\infty} \sum_{|\alpha|=k} c_{\alpha} r^{|\alpha|} W_{\alpha}^{(f)}\right) \\
& =\text { SOT }-\lim _{r \rightarrow 1} \sum_{k=0}^{\infty} \sum_{|\alpha|=k} c_{\alpha} r^{|\alpha|} \widehat{\Psi}\left(W_{\alpha}^{(f)}\right)=\text { SOT- } \lim _{r \rightarrow 1} \sum_{k=0}^{\infty} \sum_{|\alpha|=k} c_{\alpha} r^{|\alpha|} \widetilde{\varphi}_{\alpha},
\end{aligned}
$$

for any $i=1, \ldots, q$. Consequently, applying the noncommutative Berezin transform at $X \in \mathbf{D}_{g, \text { pure }}^{l}(\mathcal{H})$ on the Hardy algebra $F_{q}^{\infty}\left(\mathbf{D}_{g}^{l}\right)$, using its continuity in the strong operator topology on bounded sets and relations (6.2), (6.5), (6.4), we have

$$
\begin{aligned}
X_{i} & =\mathcal{B}_{X}\left[W_{i}^{(g)}\right]=\mathcal{B}_{X}\left[\text { SOT- } \lim _{r \rightarrow 1} \sum_{k=0}^{\infty} \sum_{|\alpha|=k} c_{\alpha} r^{|\alpha|} \widetilde{\varphi}_{\alpha}\right] \\
& =\text { SOT- } \lim _{r \rightarrow 1} \sum_{k=0}^{\infty} \sum_{|\alpha|=k} c_{\alpha} r^{|\alpha|} \mathcal{B}_{X}\left[\widetilde{\varphi}_{\alpha}\right]=\text { SOT- } \lim _{r \rightarrow 1} \sum_{k=0}^{\infty} \sum_{|\alpha|=k} c_{\alpha} r^{|\alpha|} \varphi_{\alpha}(X) \\
& =\text { SOT- } \lim _{r \rightarrow 1} \mathcal{B}_{\varphi(X)}\left[\sum_{k=0}^{\infty} \sum_{|\alpha|=k} c_{\alpha} r^{|\alpha|} W_{\alpha}^{(f)}\right] \\
& =\mathcal{B}_{\varphi(X)}[\widetilde{\xi}]=\xi_{i}(\varphi(X))
\end{aligned}
$$

for each $i=1, \ldots, q$, and any $X \in \mathbf{D}_{g, \text { pure }}^{l}(\mathcal{H})$. Hence $(\xi \circ \varphi)(X)=X$ for $X \in \mathbf{D}_{g, \text { pure }}^{l}(\mathcal{H})$. Similarly, one can prove that $(\varphi \circ \xi)(X)=X$ for $X \in \mathbf{D}_{f, \text { pure }}^{m}(\mathcal{H})$. Consequently, $\varphi: \mathbf{D}_{g, \text { pure }}^{l}(\mathcal{H}) \rightarrow \mathbf{D}_{f, \text { pure }}^{m}(\mathcal{H})$ is a bijection such that $\varphi$ and $\varphi^{-1}:=\xi$ are free holomorphic functions on the noncommutative domain $\mathbf{D}_{g, \text { rad }}^{l}(\mathcal{H})$ and $\mathbf{D}_{f, \text { rad }}^{m}(\mathcal{H})$, respectively, and have the required properties. Therefore, $\varphi \in B i h\left(\mathbf{D}_{g, \text { pure }}^{l}, \mathbf{D}_{f, \text { pure }}^{m}\right)$.

Let $\tilde{\chi} \in F_{n}^{\infty}\left(\mathbf{D}_{f}^{m}\right)$ have the representation $\sum_{\alpha \in \mathbb{F}_{n}^{+}} d_{\alpha} W_{\alpha}^{(f)}$. Then $\tilde{\chi}=$ SOT- $\lim _{r \rightarrow 1} \sum_{k=0}^{\infty} \sum_{|\alpha|=k} d_{\alpha} r^{|\alpha|} W_{\alpha}^{(f)}$. Due to relation (6.1) and the continuity of $\widehat{\Psi}$ in the strong operator topology, we have

$$
\widehat{\Psi}(\widetilde{\chi})=\text { SOT }-\lim _{r \rightarrow 1} \sum_{k=0}^{\infty} \sum_{|\alpha|=k} d_{\alpha} r^{|\alpha|} \widetilde{\varphi}_{\alpha} .
$$

Consequently, applying the noncommutative Berezin transform at $X \in \mathbf{D}_{g, \text { pure }}^{l}(\mathcal{H})$, using its SOTcontinuity on bounded sets, and relation (6.2), we get

$$
\begin{aligned}
{[\Psi(\chi)](X) } & =\mathcal{B}_{X}[\widehat{\Psi}(\widetilde{\chi})]=\text { SOT- } \lim _{r \rightarrow 1} \sum_{k=0}^{\infty} \sum_{|\alpha|=k} d_{\alpha} r^{|\alpha|} \mathcal{B}_{X}\left[\widetilde{\varphi}_{\alpha}\right] \\
& =\text { SOT- } \lim _{r \rightarrow 1} \sum_{k=0}^{\infty} \sum_{|\alpha|=k} d_{\alpha} r^{|\alpha|} \varphi_{\alpha}(X) \\
& =\text { SOT- } \lim _{r \rightarrow 1} \mathcal{B}_{\varphi(X)}\left[\sum_{k=0}^{\infty} \sum_{|\alpha|=k} d_{\alpha} r^{|\alpha|} W_{\alpha}^{(f)}\right] \\
& =\mathcal{B}_{\varphi(X)}[\widetilde{\chi}]=(\chi \circ \varphi)(X)
\end{aligned}
$$

for any $X \in \mathbf{D}_{g \text {,pure }}^{l}(\mathcal{H})$, which proves the direct implication of the theorem.

Conversely, assume that $\varphi \in B i h\left(\mathbf{D}_{g, \text { pure }}^{l}, \mathbf{D}_{f, \text { pure }}^{m}\right)$ and $\widetilde{\varphi}$ is unitarily equivalent to the universal model $W^{(f)}:=\left(W_{1}^{(f)}, \ldots, W_{n}^{(f)}\right)$. Define $\Psi(\chi):=\chi \circ \varphi, \quad \chi \in H^{\infty}\left(\mathbf{D}_{f, \text { rad }}^{m}\right)$. First note that, due to Theorem 3.5, $\chi \circ \varphi \in H^{\infty}\left(\mathbf{D}_{g, \text { rad }}^{l}\right)$ for all $\chi \in H^{\infty}\left(\mathbf{D}_{f, \text { rad }}^{m}\right)$, and $\Psi$ is a well-defined completely contractive 
homomorphism. A similar result can be obtained for the map $\Lambda(\zeta):=\zeta \circ \varphi^{-1}, \zeta \in H^{\infty}\left(\mathbf{D}_{g, \text { rad }}^{l}\right)$. Now, for any $\chi \in H^{\infty}\left(\mathbf{D}_{f, \text { rad }}^{m}\right)$ and $X \in \mathbf{D}_{f, \text { pure }}^{m}(\mathcal{H})$, we have $(\Lambda \circ \Psi)(\chi)=(\chi \circ \varphi) \circ \varphi^{-1}$ and

$$
\begin{aligned}
{\left[(\chi \circ \varphi) \circ \varphi^{-1}\right](X) } & =(\chi \circ \varphi)\left(\varphi^{-1}(X)\right)=\text { SOT- } \lim _{r \rightarrow 1} \chi\left(r \varphi_{1}\left(\varphi^{-1}(X)\right), \ldots, r \varphi_{n}\left(\varphi^{-1}(X)\right)\right) \\
& =\text { SOT- } \lim _{r \rightarrow 1} \chi\left(r X_{1}, \ldots, r X_{n}\right)=\chi\left(X_{1}, \ldots, X_{n}\right) .
\end{aligned}
$$

Hence, $\Lambda \circ \Psi=i d$. Similarly, we can show that $\Psi \circ \Lambda=i d$ and deduce that $\Psi$ is a completely isometric isomorphism. Due to Theorem 3.5, we have

$$
\widehat{\Psi}(\widetilde{\chi})=\widetilde{\chi \circ \varphi}=\mathcal{B}_{\widetilde{\varphi}}[\tilde{\chi}]=K_{f, \widetilde{\varphi}}^{(m)^{*}}\left(\widetilde{\chi} \otimes I_{\mathcal{D}_{f, m, \tilde{\varphi}}}\right) K_{f, \widetilde{\varphi}}^{(m)}, \quad \tilde{\chi} \in F_{n}^{\infty}\left(\mathbf{D}_{f}^{m}\right) .
$$

Now, we show that the Berezin kernel $K_{f, \widetilde{\varphi}}^{(m)}$ is a unitary operator. Recall that the noncommutative Berezin kernel $K_{f, W^{(f)}}^{(m)}: F^{2}\left(H_{n}\right) \rightarrow F^{2}\left(H_{n}\right) \otimes \mathcal{D}_{f, m, W^{(f)}}$ is defined by

$$
K_{f, W^{(f)}}^{(m)} \ell=\sum_{\alpha \in \mathbb{F}_{n}^{+}} \sqrt{b_{\alpha}^{(m)}} e_{\alpha} \otimes \Delta_{f, m, W^{(f)}} W_{\alpha}^{(f)^{*}} \ell, \quad \ell \in F^{2}\left(H_{n}\right),
$$

where $\mathcal{D}_{f, m, W^{(f)}}:=\overline{\Delta_{f, m, W^{(f)}}\left(F^{2}\left(H_{n}\right)\right)}$. Since $\Delta_{f, m, W^{(f)}}^{2}=\left(i d-\Phi_{f, W^{(f)}}\right)^{m}(I)=P_{\mathbb{C}}$ (see Section 1), we deduce that $\operatorname{dim} \mathcal{D}_{f, m, W^{(f)}}=1$, so that we can identify the space $\mathcal{D}_{f, m, W^{(f)}}$ with $\mathbb{C}$. Under this identification and using relation (1.4), simple computations reveal that $K_{f, W(f)}^{(m)}=I_{F^{2}\left(H_{n}\right)}$. Since $\widetilde{\varphi}$ is unitarily equivalent to the universal model $W^{(f)}:=\left(W_{1}^{(f)}, \ldots, W_{n}^{(f)}\right)$, one can easily see that the Berezin kernel $K_{f, \widetilde{\varphi}}^{(m)}$ is a unitary operator. Therefore, due to relation (․6.6), $\widehat{\Psi}$ is a unitarily implemented isomorphism. The proof is complete.

We remark that when $m=1$, we can use the functional model for pure $n$-tuples of operators in $\mathbf{D}_{f}^{1}(\mathcal{H})$ in terms of characteristic functions (see Theorem 3.26 from [29]), to deduce that $\tilde{\varphi}$ is unitarily equivalent to the universal model $W^{(f)}:=\left(W_{1}^{(f)}, \ldots, W_{n}^{(f)}\right)$ if and only if $\widetilde{\varphi}$ is a pure $n$-tuple, i.e., $\mathrm{SOT}-\lim _{k \rightarrow \infty} \Phi_{f, \widetilde{\varphi}}^{k}(I)=0$, with $\operatorname{rank}\left[I-\Phi_{f, \widetilde{\varphi}}(I)\right]=1$ and the characteristic function $\Theta_{\tilde{\varphi}}=0$.

The group of all unitarily implemented automorphisms of the Hardy algebra $H^{\infty}\left(\mathbf{D}_{f, \text { rad }}^{m}\right)$ is denoted by $A u t_{u}\left(H^{\infty}\left(\mathbf{D}_{f, \text { rad }}^{m}\right)\right)$. Let $A u t_{w}\left(\mathbf{D}_{f, \text { pure }}^{m}\right)$ be the set of all $\varphi \in \operatorname{Bih}\left(\mathbf{D}_{f, \text { pure }}^{m}, \mathbf{D}_{f, \text { pure }}^{m}\right)$ such that $\widetilde{\varphi}$ is unitarily equivalent to the universal model $W^{(f)}:=\left(W_{1}^{(f)}, \ldots, W_{n}^{(f)}\right)$. Using Theorem 2.1 and some results concerning the composition of pure free holomorphic functions (see Lemma 3.4 and Theorem 3.5), one can easily show that $\left.A u t_{w}\left(\mathbf{D}_{f, \text { pure }}^{m}\right)\right)$ is a group with respect to the composition. As a consequence of Theorem 6.2 we deduce that

$$
A u t_{u}\left(H^{\infty}\left(\mathbf{D}_{f, \text { rad }}^{m}\right)\right) \simeq A u t_{w}\left(\mathbf{D}_{f, \text { pure }}^{m}\right) .
$$

A closer look at Theorem 6.2 reveals that one can easily obtain a version of it for noncommutative domain algebras. In this case, $\varphi \in B i h_{w}\left(\mathbf{D}_{g}^{l}, \mathbf{D}_{f}^{m}\right)$, the set of all $\psi \in B i h\left(\mathbf{D}_{g}^{l}, \mathbf{D}_{f}^{m}\right)$ such that $\widetilde{\psi}$ is unitarily equivalent to the universal model $W^{(f)}:=\left(W_{1}^{(f)}, \ldots, W_{n}^{(f)}\right)$. The proof is basically the same but uses some parts from the proof of Theorem [5.1 Setting $\left.A u t_{w}\left(\mathbf{D}_{f}^{m}\right)\right):=B i h_{w}\left(\mathbf{D}_{f}^{m}, \mathbf{D}_{f}^{m}\right)$, we can deduce that

$$
A u t_{u}\left(A\left(\mathbf{D}_{f, \mathrm{rad}}^{m}\right)\right) \simeq A u t_{w}\left(\mathbf{D}_{f}^{m}\right) .
$$

Note that due to our remark above, when $m=1$, the group $A_{u} t_{w}\left(\mathbf{D}_{f}^{1}\right)$ consists of all free biholomorphic functions $\varphi \in \operatorname{Aut}\left(\mathbf{D}_{f}^{1}\right)$ with the property that the model boundary function $\widetilde{\varphi}$ is a pure $n$-tuple with $\operatorname{rank}\left[I-\Phi_{f, \widetilde{\varphi}}(I)\right]=1$ and the characteristic function $\Theta_{\widetilde{\varphi}}=0$.

We mention that in the particular case when $m=1$ and $f=X_{1}+\cdots+X_{n}$ we have a more precise result. Davidson and Pitts ([7]) used Voiculescu's ([35]) group of automorphisms of the Cuntz-Toeplitz algebra $C^{*}\left(S_{1}, \ldots, S_{n}\right)([6])$, to show (among other things) that the subgroup $A u t_{u}\left(F_{n}^{\infty}\right)$ of unitarily implemented automorphisms of the noncommutative analytic Toeplitz algebra $F_{n}^{\infty}$ is isomorphic with $\operatorname{Aut}\left(\mathbb{B}_{n}\right)$. In [30, we obtained a new proof of their result, using noncommutative Poisson transforms [21, and showed that the unitarily implemented automorphisms of the noncommutative disc algebra $\mathcal{A}_{n}$ and 
the algebra $F_{n}^{\infty}$, respectively, are determined by the free holomorphic automorphisms of $\left[B(\mathcal{H})^{n}\right]_{1}$, via the noncommutative Poisson transform. According to [7] and [30, we have

$$
\operatorname{Aut}\left(\left[B(\mathcal{H})^{n}\right]_{1}\right) \simeq \operatorname{Aut}\left(\mathbb{B}_{n}\right) \simeq \operatorname{Aut}_{u}\left(\mathcal{A}_{n}\right) \simeq \operatorname{Aut}_{u}\left(F_{n}^{\infty}\right) .
$$

We remark here that the conformal automorphisms of $\mathbb{B}_{n}$ also occur in the work of Muhly and Solel ([15]) concerning the automorphisms of Hardy algebras associated with $W^{*}$-correspondence over von Neumann algebras ([13], 14]).

\section{REFERENCES}

[1] J. AgLer, Hypercontractions and subnormality, J. Operator Theory 13 (1985), 203-217.

[2] A. Arias and F. LATrÉmolière, Isomorphisms on non-commutative domain algebras, preprint.

[3] F.A. Berezin, Covariant and contravariant symbols of operators, (Russian), Izv. Akad. Nauk. SSSR Ser. Mat. 36 (1972), 1134-1167.

[4] W.M. Bоотнвy, An introduction to differentiable manifolds and Riemannian geometry, Second edition, Pure and Applied Mathematics, 120. Academic Press, Inc., Orlando, FL, 1986. xvi+430 pp.

[5] H. Cartan, Les fonctions de deux variables complexes et le problème de la représentation analytique, J. de Math. Pures et Appl. 96 (1931), 1-114.

[6] J. Cuntz, Simple $C^{*}$-algebras generated by isometries, Commun. Math. Phys. 57 (1977), 173-185.

[7] K. R. Davidson and D.R. PitTs, The algebraic structure of non-commutative analytic Toeplitz algebras, Math. Ann. 311 (1998), 275-303.

[8] E.G. Effros and Z.J. Ruan, Operator spaces, London Mathematical Society Monographs. New Series, 23. The Clarendon Press, Oxford University Press, New York, 2000.

[9] J.W. Helton, I. Klep, S. McCullough, and N. Slingled, Noncommutative ball maps, J. Funct. Anal. 257 (2009), 47-87.

[10] K. Hoffman, Banach Spaces of Analytic Functions, Englewood Cliffs: Prentice-Hall, 1962.

[11] A.V. Isaev and S.G. Krantz, Domains with non-compact automorphism group: a survey, Adv. Math. 146 (1999), $1-38$.

[12] S.G. Krantz, Function theory of several complex variables. Reprint of the 1992 edition. AMS Chelsea Publishing, Providence, RI, 2001. xvi+564 pp.

[13] P.S. Muhly and B. Solel, Tensor algebras over $C^{*}$-correspondences: representations, dilations, and $C^{*}$-envelopes, $J$. Funct. Anal. 158 (1998), 389-457.

[14] P.S. Muhly and B. Solel, Hardy algebras, $W^{*}$-correspondences and interpolation theory, Math. Ann. 330 (2004), 353-415.

[15] P.S. Muhly and B. Solel, Schur Class Operator Functions and Automorphisms of Hardy Algebras, Documenta Math. 13 (2008), 365-411.

[16] V.I. Paulsen, Completely Bounded Maps and Dilations, Pitman Research Notes in Mathematics, Vol.146, New York, 1986.

[17] G. Pisier, Similarity Problems and Completely Bounded Maps, Springer Lect. Notes Math., Vol.1618, Springer-Verlag, New York, 1995.

[18] G. Popescu, Characteristic functions for infinite sequences of noncommuting operators, J. Operator Theory 22 (1989), $51-71$.

[19] G. Popescu, Von Neumann inequality for $\left(B(H)^{n}\right)_{1}$, Math. Scand. 68 (1991), 292-304.

[20] G. PopesCu, Functional calculus for noncommuting operators, Michigan Math. J. 42 (1995), 345-356.

[21] G. Popescu, Poisson transforms on some $C^{*}$-algebras generated by isometries, J. Funct. Anal. 161 (1999), 27-61.

[22] G. Popescu, Curvature invariant for Hilbert modules over free semigroup algebras, Adv. Math. 158 (2001), 264-309.

[23] G. Popescu, Free holomorphic functions on the unit ball of $B(\mathcal{H})^{n}$, J. Funct. Anal. 241 (2006), 268-333.

[24] G. Popescu, Noncommutative Berezin transforms and multivariable operator model theory, J. Funct. Anal. 254 (2008), 1003-1057.

[25] G. Popescu, Free holomorphic functions and interpolation, Math. Ann. 342 (2008), 1-30.

[26] G. Popescu, Free pluriharmonic majorants and commutant lifting, J. Funct. Anal. 255 (2008), 891-939.

[27] G. Popescu, Noncommutative transforms and free pluriharmonic functions, Adv. Math. 220 (2009), 831-893.

[28] G. Popescu, Noncommutative hyperbolic geometry on the unit ball of $B(\mathcal{H})^{n}$, J. Funct. Anal. 256 (2009), 4030-4070.

[29] G. Popescu, Operator theory on noncommutative domains, Mem. Amer. Math. Soc. 205 (2010), no. 964, vi+124.

[30] G. Popescu, Free holomorphic automorphisms of the unit ball of $B(\mathcal{H})^{n}$, J. Reine Angew. Math. 638 (2010).

[31] G. Popescu, Free holomorphic functions on the unit ball of $B(\mathcal{H})^{n}$,II, J. Funct. Anal. 258 (2010), $1513-1578$.

[32] T. Sunada, Holomorphic equivalence problem for bounded Reinhardt domains, Math. Ann. 235 (1978), no.2, 111-128.

[33] B. Sz.-Nagy And C. Foiaş, Harmonic Analysis of Operators on Hilbert Space, North Holland, New York 1970.

[34] P. Thullen, Zu den Abbildunden durch analytische Funktionen mehrerer komplexer Veränderlichen Die Invarianz des Mittelpunktes von Kreiskörpern, Math. Ann. 104 (1931), no.1, 244-259.

[35] D. Voiculescu, Symmetries of some reduced free product $C^{*}$-algebras, Lecture Notes in Math. 1132, 556-588, Springer Verlag, New York, 1985. 
[36] D.V. Voiculescu Free analysis questions II: The Grassmannian completion and the series expansions at the origin, preprint.

[37] J. von Neumann, Eine Spectraltheorie für allgemeine Operatoren eines unitären Raumes, Math. Nachr. 4 (1951), $258-281$.

Department of Mathematics, The University of Texas at San Antonio, San Antonio, TX 78249, USA

E-mail address: gelu.popescu@utsa.edu 